\title{
DEFORMATIONS OF COISOTROPIC SUBMANIFOLDS AND STRONG HOMOTOPY LIE ALGEBROIDS
}

\author{
YONG-GEUN OH AND JAE-SUK PARK
}

\begin{abstract}
In this paper, we study deformations of coisotropic submanifolds in a symplectic manifold. First we derive the equation that governs $C^{\infty}$ deformations of coisotropic submanifolds and define the corresponding $C^{\infty}$-moduli space of coisotropic submanifolds modulo the Hamiltonian isotopies. This is a non-commutative and non-linear generalization of the well-known description of the local deformation space of Lagrangian submanifolds as the set of graphs of closed one forms in the Darboux-Weinstein chart of a given Lagrangian submanifold. We then introduce the notion of strong homotopy Lie algebroid (or $L_{\infty}$-algebroid) and associate a canonical isomorphism class of strong homotopy Lie algebroids to each pre-symplectic manifold $(Y, \omega)$ and identify the formal deformation space of coisotropic embeddings into a symplectic manifold in terms of this strong homotopy Lie algebroid. The formal moduli space then is provided by the gauge equivalence classes of solutions of a version of the Maurer-Cartan equation (or the master equation) of the strong homotopy Lie algebroid, and plays the role of the classical part of the moduli space of quantum deformation space of coisotropic $A$-branes. We provide a criterion for the unobstructedness of the deformation problem and analyze a family of examples that illustrates that this deformation problem is obstructed in general and heavily depends on the geometry and dynamics of the null foliation.
\end{abstract}

\section{Contents}

1. Introduction

2. Geometry of coisotropic Grassmanians

3. Canonical svmplectic neighborhoods $\quad 7$

4. Leaf space connection and curvature 9

5. Geometry of the null foliation 16

6. Geometry of the svmplectic thickening 19

7. Master equation in coordinates 22

8. Hamiltonian equivalence 25

9. Strong homotopv Lie algebroid 27

The first named author is partially supported by the NSF grant \#DMS 0203593, a grant of the 2000 Korean Young Scientist Prize, and the Vilas Research Award of the University of Wisconsin. 


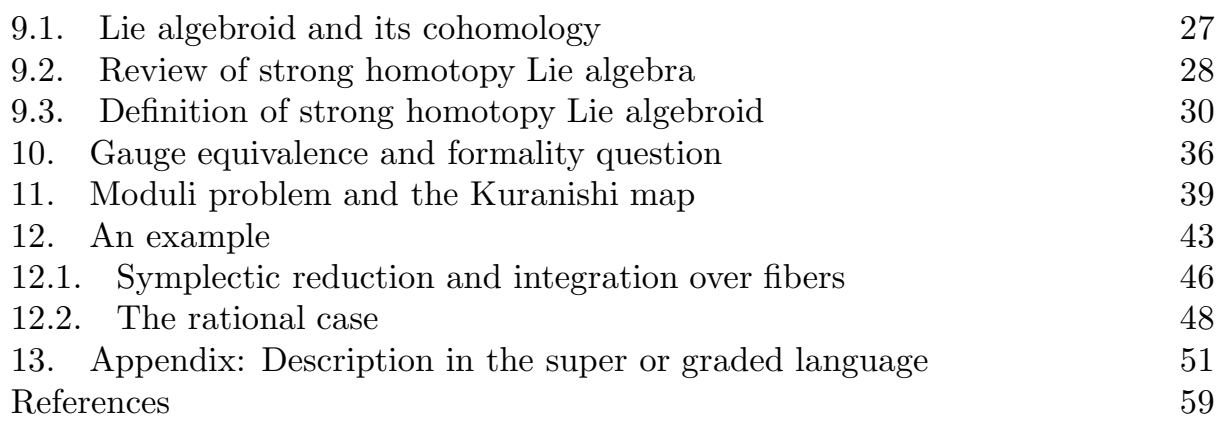

\section{INTRODUCTION}

The well-known Darboux-Weinstein theorem [We1] states that a neighborhood of any Lagrangian submanifold $L$ of any symplectic manifold $\left(X, \omega_{X}\right)$ (with real dimensions $2 n$ ) is diffeomorphic to a neighborhood of the zero section of the cotangent bundle $T^{*} L$ with the standard symplectic form

$$
\omega=-d \theta, \quad \theta=\sum_{i=1}^{n} p_{i} d q^{i}
$$

where $\theta$ is the canonical one form defined by

$$
\theta_{p}(\xi)=p(T \pi(\xi))
$$

for $p \in T^{*} L, \xi \in T_{p}\left(T^{*} L\right)$ and $\pi: T^{*} L \rightarrow L$ is the canonical projection. Furthermore it is also well-known that for any section $\alpha: L \rightarrow T^{*} L$ of $T^{*} L$, i.e., for any one form $\alpha$ on $L$, we have the identity

$$
\alpha^{*} \theta=\alpha .
$$

From this it follows that any Lagrangian submanifold $C^{1}$-close to the zero section is the graph of a closed one form. And two such Lagrangian graphs of $\alpha$ and $\alpha^{\prime}$ are Hamiltonian isotopic if and only if $\alpha-\alpha^{\prime}=d \beta$, i.e, exact. Therefore the (local) moduli space near the given Lagrangian submanifold $L \subset\left(X, \omega_{X}\right)$ is diffeomorphic to a neighborhood of $0 \in H^{1}(L ; \mathbb{R})$. In particular the local moduli problem of the Lagrangian submanifold up to Hamiltonian isotopy is linear and commutative. It depends only on the manifold $L$ but is independent of where the abstract manifold $L$ is embedded into as a Lagrangian submanifold.

The main purpose of the present paper is to describe the moduli space of coisotropic submanifolds modulo the Hamiltonian isotopy in a symplectic manifold, and its formal counterpart. Recall that a submanifold $i: Y \hookrightarrow\left(X, \omega_{X}\right)$ is called coisotropic if the symplectic orthogonal $(T Y)^{\omega}$ satisfies

$$
(T Y)^{\omega} \subset T Y
$$

and has constant rank. Then the pull-back $\omega=i^{*} \omega_{X}$ is a closed two form with constant rank $2 k$ on $Y$. Such a pair $(Y, \omega)$ in general is called a pre-symplectic manifold. Gotay $[\mathrm{Go}]$ proved that any given pre-symplectic manifold $(Y, \omega)$ can be embedded into a symplectic manifold $\left(X, \omega_{X}\right)$ as a coisotropic submanifold so that $i^{*} \omega_{X}=\omega$. Furthermore the neighborhoods of any two such embeddings are diffeomorphic regardless of $\left(X, \omega_{X}\right)$. In fact, a neighborhood of any coisotropic 
submanifold $Y \subset\left(X, \omega_{X}\right)$ is locally diffeomorphic to a neighborhood of the zero section $o_{E^{*}} \cong Y$ of the bundle

$$
E^{*} \rightarrow Y ; \quad E=(T Y)^{\omega} \subset T Y
$$

with a symplectic form $\omega_{E^{*}}$ defined in the neighborhood. One drawback of Gotay's theorem [Go] is that it does not provide the symplectic form $\omega_{E^{*}}$ explicitly. This hindered our study of the current deformation problem in the beginning. We would like to recall that in the Lagrangian case we have the explicit canonical symplectic form $\omega_{X}=-d \theta$ on the model space $T^{*} L$ which enables one to study the moduli problem explicitly. See section 3 for more comments on this point.

We first note that $E=T \mathcal{F}$ is the tangent bundle of the null foliation of $(Y, \omega)$ and so $E^{*}$ is the cotangent bundle $T^{*} \mathcal{F}$ of the foliation $\mathcal{F}$. Then we introduce an explicitly given one form $\theta_{G}$ on $T^{*} \mathcal{F}$ which is the analogue to the canonical one form $\theta$ on the cotangent bundle of the manifold. This one form will, however, depend on the choice of the splitting

$$
T Y=G \oplus E .
$$

This splitting can be regarded as a "connection" of the "E-bundle" $T Y \rightarrow Y / \sim$ where $Y / \sim$ is the space of leaves of the null foliation of $Y$. Using this one form $\theta_{G}$ we will write down the explicit symplectic form $\omega_{E^{*}}$ in terms of $(Y, \omega)$ and $\theta_{G}$. And then we will write down the defining equation for a section $s$ of $\pi: E^{*} \rightarrow Y$ whose graph is to be coisotropic in $\left(E^{*}, \omega_{E^{*}}\right)$ (in a neighborhood of the zero section). The moduli problem for this general coisotropic case is non-commutative and fully nonlinear. The moduli problem becomes trivial when the foliation is one dimensional (i.e., the case of hypersurfaces) and becomes quadratic when the null-foliation also allows a transverse foliation. The moduli problem up to Hamiltonian isotropy is obstructed in general.

In fact, it turns out that the equation for the formal moduli problem involves the structure of a strong homotopy Lie-algebra

$$
\left(\Omega^{\bullet}(\mathcal{F}), \mathfrak{m}\right),
$$

where $\mathfrak{m}=\left\{\mathfrak{m}_{i}\right\}_{i=1}^{\ell}, \Omega^{\bullet}(\mathcal{F}):=\oplus_{\ell=0}^{n-k} \Gamma\left(\Lambda^{\ell}\left(E^{*}\right)\right)$ : In general, we call a Lie algebroid $E \rightarrow Y$ a strong homotopy Lie algebroid if its associated graded group $\Omega^{\bullet}(E):=$ $\oplus \Gamma\left(\Lambda^{\ell}\left(E^{*}\right)\right)$ has the structure of strong homotopy Lie algebra (or $L_{\infty}$-algebra) where $\Lambda^{\ell}\left(E^{*}\right)$ is the set of $\ell$-wedge product of $E^{*}$ so that $\mathfrak{m}_{1}$ is the $E$-differential induced by the Lie algebroid structure on $E \rightarrow Y$. In our case, $\mathfrak{m}_{1}$ is the leafwise differential associated to the null foliation and $\mathfrak{m}_{2}=\{\cdot, \cdot\}$ a (graded) bilinear map, which is an analog to a Poisson bracket but not satisfying the Jacobi identity whose failure is then measured by $\mathfrak{m}_{3}$ and so on. We refer to later sections, especially section 9 for precise details.

The main purpose of the present paper is to unravel the geometric and algebraic structures that govern the deformation problem of coisotropic submanifolds. It turns out that even setting up the proper framework for the study of this deformation problem requires definitions of many new geometric notions which have not appeared in the literature before, as far as we know. We have discovered them first via tensor calculations using coordinates and then associated the relevant geometric structures to them. Partly because of this and also because the coordinate calculations are better suited for the super-calculus in the Batalin-Vilkovisky formalism as outlined in the appendix, we prefer to carry out the coordinate calculations first and then provide the corresponding invariant descriptions. However the latter can 
be done only after one develops an appropriate invariant calculus in the properly formulated geometric framework. It appears that a full systematic study of those should be a theme of separate study which is not our main interest in the present paper. In the present paper, we refrain from developing the full invariant calculus, but carry out only those essential for a self-contained description of our deformation problem of coisotropic submanifolds. A more thorough study of the invariant calculus in the context of general foliation theory will be made elsewhere.

The present work is a mathematical spin-off of our paper $[\mathrm{OP}]$ in preparation in which we provide an off-shell description of topological open/closed $A$-strings and $A$-branes on symplectic manifold ("bulk"). In [OP], we have derived in the natural framework of the Batalin-Vilkovisky formalism that the set of $A$-branes of the topological open $\sigma$-model on the symplectic manifold is the set of coisotropic submanifolds (at least the bosonic part thereof), which we also outline in the appendix of the present paper. A possible significance of coisotropic branes in homological mirror symmetry was first observed by Kapustin and Orlov [KaOr]. After this paper was originally submitted and circulated, a paper [CF2] by Catteneo and Felder has appeared in which a similar discussion on the role of coisotropic submanifolds in the context of Poisson manifolds is carried out in a same spirit to ours given in Appendix in relation to the open string $\sigma$-model. There has also appeared a paper $[\mathrm{R}]$ by W.-D. Ruan in which Ruan studies a deformation problem of a restricted class of coisotropic submanifolds which he calls integral coisotropic submanifolds: a coisotropic submanifold $Y \subset(X, \omega)$ is said to be integral if its associated null foliation is defined by a smooth fibration $\pi: Y \rightarrow S$ over a smooth (Hausdorff) manifold $S$. He proved that deformations inside the class of integral coisotropic submanifolds are unobstructed and the moduli space thereof modulo Hamiltonian diffeomorphisms is smooth and finite dimensional [R].

This research was initiated while both authors were visiting the Korea Institute for Advanced Study in the winter of 2002. We thank KIAS for its financial support and excellent research environment. The first named author thanks M. Zambon for explaining the example mentioned in section [1] in an IPAM conference in April, 2003 and for asking some questions on the preliminary version of this paper. He himself independently studied neighborhoods of coisotropic submanifolds [Za] and found an example of a coisotropic submanifold which illustrates the fact that $C^{1}$ close coisotropic submanifolds of the same nullity do not form a Fréchet manifold in general.

The second named author thanks the mathematics department of POSTECH, especially to his host B. Kim, for the financial support and excellent research environment, and to the mathematics department of University of the WisconsinMadison for its hospitality during his visit. We also thank J. Stasheff for some historical comments on the $L_{\infty}$-structure and the Gerstenhaber bracket, and I. Vaisman for attracting our attention to his paper [V] after the paper was submitted. The first named author also thanks N. Kieserman for pointing out and correcting some inaccuracy in our coordinate calculations. Last, but not least, we are very much indebted to the referees for making many valuable suggestions and corrections which have led to much improvement and clarification of the contents and presentation of the paper. 


\section{Geometry of Coisotropic Grassmanians}

In this section, we will summarize some linear algebraic facts on the coisotropic subspace $C$ (with real dimensions $n+k$ where $0 \leq k \leq n$ ) in $\mathbb{C}^{n}$ with respect to the standard symplectic form $\omega=\omega_{0, n}$. We denote by $C^{\omega}$ the $\omega$-orthogonal complement of $C$ in $\mathbb{R}^{2 n}$ and by $\Gamma_{k}$ the set of coisotropic subspaces of $\left(\mathbb{R}^{2 n}, \omega\right)$. In other words,

$$
\Gamma_{k}=\Gamma_{k}\left(\mathbb{R}^{2 n}, \omega\right)=:\left\{C \in G r_{n+k}\left(\mathbb{R}^{2 n}\right) \mid C^{\omega} \subset C\right\} .
$$

From the definition, we have the canonical flag,

$$
0 \subset C^{\omega} \subset C \subset \mathbb{R}^{2 n}
$$

for any coisotropic subspace. We call $\left(C, C^{\omega}\right)$ a coisotropic pair. Combining this with the standard complex structure on $\mathbb{R}^{2 n} \cong \mathbb{C}^{n}$, we have the splitting

$$
C=H_{C} \oplus C^{\omega}
$$

where $H_{C}$ is the complex subspace of $C$.

Proposition 2.1. Let $0 \leq k \leq n$ be fixed. The unitary group $U(n)$ acts transitively on $\Gamma_{k}$. The corresponding homogeneous space is given by

$$
\Gamma_{k} \cong U(n) / U(k) \times O(n-k)
$$

where $U(k) \times O(n-k) \subset U(n)$ is the isotropy group of the coisotropic subspace $\mathbb{C}^{k} \oplus \mathbb{R}^{n-k} \subset \mathbb{C}^{n}$. In particular we have

$$
\operatorname{dim} \Gamma_{k}\left(\mathbb{R}^{2 n}, \omega\right)=\frac{(n+3 k+1)(n-k)}{2} .
$$

Proof. Let $C \subset \mathbb{C}^{n}$ be a coisotropic subspace with rank $2 k$ and $C^{\omega}$ be its null space. Since $C^{\omega} \subset C$, it follows

$$
g\left(C, i C^{\omega}\right)=0,
$$

where $g$ is the Euclidean inner product. Now if we write

$$
H_{C}=\left\{x \in C \mid g\left(x, C^{\omega}\right)=0\right\},
$$

then $H_{C}$ is the Hermitian orthogonal complement of $C^{\omega}$ which is a complex subspace. Similarly $C^{\omega} \oplus i C^{\omega}$ is also a complex subspace. Therefore we have obtained the Hermitian orthogonal decomposition

$$
\mathbb{C}^{n}=H_{C} \oplus\left(C^{\omega} \oplus i C^{\omega}\right) .
$$

It then follows that there is a unitary matrix $A \in U(n)$ such that $C=A \cdot\left(\mathbb{C}^{k} \oplus\right.$ $\left.\mathbb{R}^{n-k}\right)$. This proves that $U(n)$ acts transitively on $\Gamma_{k}$. Now it is enough to show that the isotropy group of $\mathbb{C}^{k} \oplus \mathbb{R}^{n-k}$ is $U(k) \times O(n-k) \subset U(n)$ which is obvious. This proves the proposition.

Next we give a parametrization of all the coisotropic subspaces near given $C \in$ $\Gamma_{k}$. Up to the unitary change of coordinates we may assume that $C$ is the canonical model

$$
C=\mathbb{C}^{k} \oplus \mathbb{R}^{n-k} .
$$

We denote the (Euclidean) orthogonal complement of $C$ by $C^{\perp}=i \mathbb{R}^{n-k}$ which is canonically isomorphic to $\left(C^{\omega}\right)^{*}$ via the isomorphism $\widetilde{\omega}: \mathbb{C}^{n} \rightarrow\left(\mathbb{C}^{n}\right)^{*}$. Then any nearby subspace of dimension $\operatorname{dim} C$ that is transverse to $C^{\perp}$ can be written as the graph of the linear map

$$
A: C \rightarrow C^{\perp} \cong\left(C^{\omega}\right)^{*}
$$


i.e., has the form

$$
C_{A}:=\left\{(x, A x) \in C \oplus C^{\perp}=\mathbb{R}^{2 n} \mid x \in C\right\} .
$$

Denote $A=A_{H} \oplus A_{I}$ where

$$
\begin{aligned}
& A_{H}: H=\mathbb{C}^{k} \rightarrow C^{\perp} \cong\left(C^{\omega}\right)^{*}, \\
& A_{I}: C^{\omega}=\mathbb{R}^{n-k} \rightarrow C^{\perp} \cong\left(C^{\omega}\right)^{*} .
\end{aligned}
$$

Note that the symplectic form $\omega$ induce the canonical isomorphism

$$
\begin{aligned}
\widetilde{\omega}^{H} & : \mathbb{C}^{k} \rightarrow\left(\mathbb{C}^{k}\right)^{*} \\
\widetilde{\omega}^{I} & : \mathbb{R}^{n-k}=C^{\omega} \rightarrow\left(C^{\omega}\right)^{*} \cong C^{\perp}=i \mathbb{R}^{n-k}
\end{aligned}
$$

With this identification, the symplectic form $\omega$ has the form

$$
\omega=\pi^{*} \omega_{0, k}+\sum_{i=1}^{n-k} d x_{i} \wedge d y^{i},
$$

where $\pi: \mathbb{C}^{n} \rightarrow \mathbb{C}^{k}$ is the projection and $\left(x_{1}, \cdots, x_{n-k}\right)$ the standard coordinates of $\mathbb{R}^{n-k}$ and $\left(y^{1}, \cdots, y^{n-k}\right)$ its dual coordinates of $\left(\mathbb{R}^{n-k}\right)^{*}$. We also denote by $\pi_{H}:\left(\mathbb{C}^{k}\right)^{*} \rightarrow \mathbb{C}^{k}$ the inverse of the above mentioned canonical isomorphism $\widetilde{\omega}^{H}$. Then we have the following

Proposition 2.2. The subspace $C_{A}$ is coisotropic if and only if $A_{H}$ and $A_{I}$ satisfies

$$
A_{I}-\left(A_{I}\right)^{*}+A_{H} \pi_{H}\left(A_{H}\right)^{*}=0 .
$$

Proof. We need to study under what conditions on $A_{H}$ and $A_{I}$, the relation $\left(C_{A}\right)^{\omega} \subset$ $C_{A}$ holds and vice versa. Let $\left(\xi_{H}, \xi_{I}, \xi_{I}^{*}\right) \in\left(C_{A}\right)^{\omega} \subset \mathbb{C}^{n}=\mathbb{C}^{k} \oplus \mathbb{R}^{n-k} \oplus i \mathbb{R}^{n-k}$, i.e, let

$$
\omega\left(\left(v_{H}, v_{I}, A\left(v_{H}, v_{I}\right)\right),\left(\xi_{H}, \xi_{I}, \xi_{I}^{*}\right)\right)=0
$$

for all $\left(v_{H}, v_{I}\right) \in \mathbb{C}^{k} \oplus \mathbb{R}^{n-k}$. It follows from (2.7) and from the above identifications, we have

$$
\begin{aligned}
0 & =\omega\left(v_{H}, \xi_{H}\right)+A\left(v_{H}, v_{I}\right)\left(\xi_{I}\right)-\xi_{I}^{*}\left(v_{I}\right) \\
& =\omega\left(v_{H}, \xi_{H}\right)+\left(A_{H}\left(v_{H}\right)\left(\xi_{I}\right)+A_{I}\left(v_{I}\right)\left(\xi_{I}\right)\right)-\xi_{I}^{*}\left(v_{I}\right)
\end{aligned}
$$

for all $v_{H}, v_{I}$. Substituting $v_{I}=0$ we get

$$
\omega\left(v_{H}, \xi_{H}\right)+A_{H}\left(v_{H}\right)\left(\xi_{I}\right)=0
$$

for all $v_{H} \in \mathbb{C}^{k}$. With the above identification, we derive

$$
-\widetilde{\omega}_{0}^{H}\left(\xi_{H}\right)\left(v_{H}\right)+A_{H}^{*}\left(\xi_{I}\right)\left(v_{H}\right)=0
$$

for all $v_{H} \in \mathbb{C}^{k}$. Therefore we derive

$$
\xi_{H}=\pi_{H} A_{H}^{*}\left(\xi_{I}\right) .
$$

And substituting $v_{H}=0$, we derive

$$
A_{I}\left(v_{I}\right)\left(\xi_{I}\right)-\xi_{I}^{*}\left(v_{I}\right)=0
$$

for all $v_{I} \in \mathbb{R}^{n-k}$ and hence

$$
A_{I}^{*}\left(\xi_{I}\right)=\xi_{I}^{*} .
$$

Therefore it follows from (2.10) and (2.11) that $C_{A}$ is coisotropic if and only if

$$
A_{I}^{*}\left(\xi_{I}\right)=A\left(\pi_{H} A_{H}^{*}\left(\xi_{I}\right), \xi_{I}\right)=A_{H} \oplus A_{I}\left(\pi_{H} A_{H}^{*}\left(\xi_{I}\right), \xi_{I}\right)
$$


for all $\xi_{I}$. The latter becomes

$$
A_{I}-\left(A_{I}\right)^{*}+A_{H} \pi_{H}\left(A_{H}\right)^{*}=0,
$$

which finishes the proof.

Remark 2.1. Note that when $k=0$, this reduces to the standard parametrization of Lagrangian subspaces by the set of symmetric matrices.

\section{CANONICAL SYMPLECTIC NEIGHBORHOOdS}

We first recall some basic properties of coisotropic submanifolds and the coisotropic neighborhood theorem [Go]. We will mostly adopt the notations used in [Go]. First $(T Y)^{\omega}:=E$ defines a distribution, the so called characteristic distribution on $Y$ which is integrable since $\omega$ is closed. We call the corresponding foliation the null foliation on $Y$ and denote it by $\mathcal{F}$. The null foliation carries a natural transverse symplectic form but the space of leaves may not be a Hausdorff space in general. This space of leaves provides a symplectic invariant of coisotropic submanifolds up to the Hamiltonian isotopy, or equivalently an invariant of the pre-symplectic manifold $(Y, \omega)$. We refer to the next section for the detailed description of the geometry of null foliation.

We now consider the dual bundle $\pi: E^{*} \rightarrow Y$ of $E$. The bundle $\left.T E^{*}\right|_{Y}$ where $Y \subset E^{*}$ is the zero section of $E^{*}$ carries the canonical decomposition

$$
\left.T E^{*}\right|_{Y}=T Y \oplus E^{*} \text {. }
$$

It is easy to check that the canonical isomorphism

$$
\widetilde{\omega}: T X \rightarrow T^{*} X
$$

maps $T Y^{\omega}$ to the conormal $N^{*} Y \subset T^{*} X$, and induces an isomorphism between $N Y=T X / T Y$ and $E^{*}$. In the standard notation in the foliation theory, $E$ and $E^{*}$ are denoted by $T \mathcal{F}$ and $T^{*} \mathcal{F}$ and called the tangent bundle (respectively cotangent bundle) of the foliation $\mathcal{F}$.

Following Gotay [Go], we choose a splitting

$$
T Y=G \oplus E, \quad E=(T Y)^{\omega} .
$$

Using this splitting, we can write a symplectic form on a neighborhood of the zero section $Y \hookrightarrow E^{*}$ in the following way (see $[\mathrm{V}]$ ). We denote by

$$
p_{G}: T Y \rightarrow E
$$

the projection to $E$ along $G$ in the splitting (3.1). We have the bundle map

$$
T E^{*} \stackrel{T \pi}{\longrightarrow} T Y \stackrel{p_{G}}{\longrightarrow} E .
$$

Let $\alpha \in E^{*}$ and $\xi \in T_{\alpha} E^{*}$. We define the one form $\theta_{G}$ on $E^{*}$ by its value

$$
\theta_{G, \alpha}(\xi):=\alpha\left(p_{G} \circ T \pi(\xi)\right)
$$

at each $\alpha \in E^{*}$. Then we define the closed (indeed exact) two form on $E^{*}$ by

$$
-d \theta_{G}
$$

It is easy to see that the closed two form

$$
\omega_{E^{*}}:=\pi^{*} \omega-d \theta_{G}
$$

is non-degenerate in a neighborhood $U \subset E^{*}$ of the zero section (See the coordinate expression (6.6) of $d \theta_{G}$ and $\omega_{U}$ ). We denote the restriction of $\omega_{E^{*}}$ by $\omega_{U}$. Then the 
pair $\left(U, \omega_{U}\right)$ provides an explicit normal form of the symplectic neighborhood of the pair $(Y, \omega)$ which depends only on $(Y, \omega)$ and the splitting (3.1). By Weinstein's uniqueness theorem [We1], this normal form is unique up to diffeomorphism. We call the pair $\left(U, \omega_{U}\right)$ a (canonical) symplectic thickening of the pre-symplectic manifold $\left(Y, \omega_{Y}\right)$. We refer to Corollary 5.1 for the precise statement on the uniqueness.

Here is the coisotropic analog to (1.2) whose proof we omit.

Lemma 3.1. For any section of $s: Y \rightarrow U \subset E^{*}$, we have the identity

$$
s^{*} \theta_{G}=p_{G}^{*} s
$$

where $p_{G}^{*}: E^{*} \rightarrow T^{*} Y$ is the adjoint to the projection $p_{G}: T Y \rightarrow E$.

We next introduce morphisms between pre-symplectic manifolds and automorphisms of $(Y, \omega)$.

Definition 3.1. Let $(Y, \omega)$ and $\left(Y^{\prime}, \omega^{\prime}\right)$ be two pre-symplectic manifolds. A diffeomorphism $\phi: Y \rightarrow Y^{\prime}$ is called pre-symplectic if $\phi^{*} \omega^{\prime}=\omega$.

Lemma 3.1 immediately implies the following

Corollary 3.2. Suppose that the graph $i:$ Graph $s \hookrightarrow U$ of a section $s: Y \rightarrow U$ is a coisotropic submanifold i.e., $i^{*} \omega_{U}$ induces a pre-symplectic structure. Then the map $s:(Y, \omega) \rightarrow\left(\right.$ Graphs,$\left.i^{*} \omega_{U}\right)$ is a pre-symplectic diffeomorphism if and only if $p_{G}^{*} s$ defines a closed one form on $Y$.

Proof. We first note that

$$
s^{*}\left(i^{*} \omega_{U}\right)=s^{*}\left(\omega_{U}\right)=s^{*} \pi^{*} \omega-s^{*}\left(d \theta_{G}\right)=\omega-d s^{*} \theta_{G}=\omega-d\left(p_{G}^{*} s\right) .
$$

The corollary immediately follows from this.

We would like to emphasize that unlike Lagrangian submanifolds for which there is no intrinsic structure, coisotropic submanifolds carry an intrinsic geometric structure, the pre-symplectic form $\omega$. Therefore the hypothesis in this corollary is not an automatic proposition even when $p_{G}^{*} s$ is a closed one form on $Y$. In the next several sections, we will provide a description of the condition under which the graph of $s: Y \rightarrow U$ becomes coisotropic.

However, (3.4) shows that up to a diffeomorphism $s \circ i: Y \rightarrow \operatorname{Graph}(s)$, the presymplectic form of any nearby coisotropic submanifold is cohomologous to the given $\omega$ on $Y$. Therefore all the pre-symplectic structures that occur in the study of local deformations of a coisotropic submanifold is special in that they are cohomologous to one another up to the pull-back by diffeomorphisms.

The following question seems to be an interesting nontrivial question to ask in general.

Question 3.2. Let $(Y, \omega),\left(Y, \omega^{\prime}\right)$ be pre-symplectic structures of the same rank such that

$$
[\omega]=\left[\omega^{\prime}\right] \quad \text { in } H^{2}(Y, \mathbb{R}),
$$

i.e., $\omega^{\prime}-\omega=d \theta$ for some one form on $Y$. Suppose that $\theta$ is sufficiently $C^{\infty}$ small. Are they diffeomorphic or can they be connected by an isotopy of pre-symplectic forms of constant rank? This question is closely related to the question whether the set of coisotropic submanifolds of constant rank is locally path-connected (see $[\mathrm{Za}])$. 
Definition 3.3. Let $(Y, \omega)$ be a pre-symplectic manifold. A vector field $\xi$ on $Y$ is called or locally pre-Hamiltonian if $\left.\mathcal{L}_{\xi}(\omega)=d(\xi\rfloor \omega\right)=0$. We call $\xi$ (globally) preHamiltonian if the one form $\xi\rfloor \omega$ is exact. We call the diffeomorphisms generated by $\xi$ locally pre-Hamiltonian (respectively pre-Hamiltonian) diffeomorphisms. We call pre-symplectic any diffeomorphism $\phi$ that satisfy $\phi^{*} \omega=\omega$.

We denote by $\mathcal{P H a m}(Y, \omega)$ the set of pre-Hamiltonian diffeomorphisms and by $\mathcal{P} \operatorname{Symp}(Y, \omega)$ the set of pre-symplectic diffeomorphisms of $(Y, \omega)$. According to our definitions, the set of locally pre-Hamiltonian diffeomorphisms is the identity component of $\mathcal{P} \operatorname{Symp}(Y, \omega)$. Therefore we will denote the latter by $\mathcal{P} \operatorname{Symp}_{0}(Y, \omega)$.

The following will be important in our formulation of the moduli problem of coisotropic submanifolds later. We will give its proof in section 8

Theorem 3.3. Any locally pre-Hamiltonian (respectively, pre-Hamiltonian) vector field $\xi$ on a pre-symplectic manifold $(Y, \omega)$ can be extended to a locally Hamiltonian (respectively, Hamiltonian) vector field on the thickening $\left(U, \omega_{U}\right)$.

\section{LEAF SPACE CONNECTION AND CURVATURE}

However the one-form $\theta_{G}$ defined in the previous section depends on the splitting (3.1). We now describe this dependence more systematically. In this section and the next, we will study the intrinsic geometry of pre-symplectic manifold $(Y, \omega)$ and the extrinsic geometry of its symplectic thickening in section [6. We first need to develop some invariant calculus "over the leaf space" which will play an important role in our study of the deformation problem of coisotropic submanifolds later. This section applies to any foliation, not just to our null foliation. As far as we know, this calculus has not been introduced in the literature yet.

Let $\mathcal{F}$ be an arbitrary foliation on a smooth manifold $Y$. Following the standard notations in the foliation theory, we define the normal bundle $N \mathcal{F}$ and conormal bundle $N^{*} \mathcal{F}$ of the foliation $\mathcal{F}$ by

$$
N_{y} \mathcal{F}:=T_{y} Y / E_{y}, \quad N_{y}^{*} \mathcal{F}:=\left(T_{y} / E_{y}\right)^{*} \cong E_{y}^{\circ} \subset T_{y}^{*} Y .
$$

In this vein, we will denote $E=T \mathcal{F}$ and $E^{*}=T^{*} \mathcal{F}$ respectively, whenever it makes our discussion more transparent. We have the natural exact sequences

$$
\begin{aligned}
& 0 \rightarrow T \mathcal{F} \rightarrow T Y \rightarrow N \mathcal{F} \rightarrow 0, \\
& 0 \leftarrow T^{*} \mathcal{F} \leftarrow T^{*} Y \leftarrow N^{*} \mathcal{F} \leftarrow 0 .
\end{aligned}
$$

The choice of splitting $T Y=G \oplus T \mathcal{F}$ may be regarded as a "connection" of the " $E$-bundle" $T Y \rightarrow Y / \sim$ where $Y / \sim$ is the space of leaves of the foliation on $Y$. Note that $Y / \sim$ is not Hausdorff in general. We will indeed call a choice of splitting a leaf space connection of $\mathcal{F}$ in general.

We can also describe the splitting in a more invariant way as follows: Consider bundle maps $\Pi: T Y \rightarrow T Y$ that satisfy

$$
\Pi_{x}^{2}=\Pi_{x}, \operatorname{im} \Pi_{x}=T_{x} \mathcal{F}
$$

at every point of $Y$, and denote the set of such projections by

$$
\mathcal{A}_{E}(T Y) \subset \Gamma(\operatorname{Hom}(T Y, T Y))=\Omega_{1}^{1}(Y) .
$$

There is a one-one correspondence between the choice of splittings (3.1) and the set $\mathcal{A}_{E}(T Y)$ provided by the correspondence

$$
\Pi \leftrightarrow G:=\operatorname{ker} \Pi .
$$


If necessary, we will denote by $\Pi_{G}$ the element with ker $\Pi=G$ and by $G_{\Pi}$ the complement to $E$ determined by $\Pi$. We will use either of the two descriptions, whichever is more convenient depending on the circumstances.

The following is easy to see by using the isomorphism $\pi_{G}: G \rightarrow N \mathcal{F}$ where $\pi_{G}$ is the restriction to $G$ of the natural projection $\pi_{\Pi}: T Y \rightarrow N \mathcal{F}$. We omit its proof.

Lemma 4.1. The space of splittings [3.1) is an infinite dimensional (Frechet) manifold modelled by

$$
\Gamma\left(H o m\left(G_{0}, T \mathcal{F}\right)\right) \cong \Gamma\left(N^{*} \mathcal{F} \otimes T \mathcal{F}\right):
$$

for any reference choice $\Pi_{0}$, and for other $\Pi$, we have

$$
\begin{aligned}
& G_{\Pi}=\left\{\eta \oplus B_{\Pi_{0} \Pi} \circ \pi_{\Pi_{0}}(\eta) \in T Y \mid \eta \in G_{0}, B_{\Pi_{0} \Pi} \in \Gamma(\operatorname{Hom}(N \mathcal{F}, T \mathcal{F}))\right\} \\
& =\left\{\pi_{\Pi}^{-1}(y) \oplus B_{\Pi_{0} \Pi}(y) \in T Y \mid y \in N \mathcal{F}, B_{\Pi_{0} \Pi} \in \Gamma(\operatorname{Hom}(N \mathcal{F}, T \mathcal{F}))\right\}
\end{aligned}
$$

Moreover, it is weakly contractible.

Next we introduce the analogue of "curvature" of the above "connection". To define this, we recall some basic facts about the foliation coordinates. We can choose coordinates on $Y$ adapted to the foliation in the following way. Since the distribution $E$ is integrable, the Frobenius theorem provides coordinates

$$
\left(y^{1}, \cdots, y^{\ell}, y^{\ell+1}, \cdots, y^{m}\right)
$$

on an open subset $V \subset Y$, such that the plaques of $V$ are given by the equation

$$
y^{1}=c^{1}, \cdots, y^{\ell}=c^{\ell}, \quad c^{i} \text { s constant. }
$$

In particular, we have

$$
E_{x}=T_{x} \mathcal{F}=\operatorname{span}\left\{\frac{\partial}{\partial y^{\ell+1}}, \cdots, \frac{\partial}{\partial y^{m}}\right\} .
$$

We denote

$$
q^{\alpha}=y^{\ell+\alpha}, \quad 1 \leq \alpha \leq m-\ell .
$$

For the given splitting $T Y=G \oplus E$, we can write

$$
G_{x}=\operatorname{span}\left\{\frac{\partial}{\partial y^{i}}+\sum_{\alpha=1}^{m-\ell} R_{i}^{\alpha} \frac{\partial}{\partial q^{\alpha}}\right\}_{1 \leq i \leq \ell}
$$

for some $R_{i}^{\alpha}$ 's, which are uniquely determined by the splitting and the given coordinates. Here $R_{i}^{\alpha}$ 's can be regarded as the "Christoffel symbols" for the "connection" $\Pi$.

From now on, we will use the summation convention for repeated indices, whenever there is no danger of confusion. Roman indices run over $1, \cdots, \ell$, and Greek ones over $1, \cdots, m-\ell$.

Now we are ready to provide the definition of the "curvature" of the П-connection.

Definition 4.1. Let $\Pi \in \mathcal{A}_{E}(T Y)$ and denote by $\Pi: T Y=G_{\Pi} \oplus T \mathcal{F}$ the corresponding splitting. The transverse $\Pi$-curvature of the foliation $\mathcal{F}$ is a $T \mathcal{F}$-valued two form defined on $N \mathcal{F}$ as follows: Let $\pi: T Y \rightarrow N \mathcal{F}$ be the canonical projection and

$$
\pi_{\Pi}: G_{\Pi} \rightarrow N \mathcal{F}
$$

be the induced isomorphism. Then we define

$$
F_{\Pi}: \Gamma(N \mathcal{F}) \otimes \Gamma(N \mathcal{F}) \rightarrow \Gamma(T \mathcal{F})
$$


by

$$
F_{\Pi}\left(\eta_{1}, \eta_{2}\right):=\Pi([X, Y])
$$

where $X=\pi_{\Pi}^{-1}\left(\eta_{1}\right)$ and $Y=\pi_{\Pi}^{-1}\left(\eta_{2}\right)$ and $[X, Y]$ is the Lie bracket on $Y$.

The following proposition, which is straightforward to check, shows that $F_{\Pi}$ is a tensorial object, justifying the name transverse $\Pi$-curvature. This tensor will play a crucial role in our description of the strong homotopy Lie algebroid associated to the pre-symplectic manifold $\left(Y, \omega_{Y}\right)$ (and so of coisotropic submanifolds) and its Maurer-Cartan equation.

Proposition 4.2. Let $F_{\Pi}$ be as above. For any smooth functions $f, g$ on $Y$ and sections $\eta_{1}, \eta_{2}$ of $N \mathcal{F}$, we have the identity

$$
F_{\Pi}\left(f \eta_{1}, g \eta_{2}\right)=f g F_{\Pi}\left(\eta_{1}, \eta_{2}\right)
$$

i.e., the map $F_{\Pi}$ defines a well-defined section as an element in $\Gamma\left(\Lambda^{2}\left(N^{*} \mathcal{F}\right) \otimes T \mathcal{F}\right)$.

Proof. Let $X, Y$ be the unique lifts of $\eta_{1}, \eta_{2}$ in $\Gamma(G) \subset \Gamma(T Y)$ as in the definition. Then it follows that $f X, g Y$ are the lifts of $f \eta_{1}, g \eta_{2}$. We have

$$
[f X, g Y]=f g[X, Y]+f X[g] Y-g Y[f] X .
$$

Since $X, Y$ are tangent to $G$, we derive

$$
\Pi([f X, g Y])=f g \Pi([X, Y])
$$

which finishes the proof.

In the foliation coordinates $\left(y^{1}, \cdots, y^{\ell}, q^{1}, \cdots, q^{m-\ell}\right), F_{\Pi}$ has the expression

$$
F_{\Pi}=F_{i j}^{\beta} \frac{\partial}{\partial q^{\beta}} \otimes d y^{i} \wedge d y^{j} \in \Gamma\left(\Lambda^{2}\left(N^{*} \mathcal{F}\right) \otimes T \mathcal{F}\right),
$$

where

$$
F_{i j}^{\beta}=\frac{\partial R_{j}^{\beta}}{\partial y^{i}}-\frac{\partial R_{i}^{\beta}}{\partial y^{j}}+R_{i}^{\gamma} \frac{\partial R_{j}^{\beta}}{\partial q^{\gamma}}-R_{j}^{\gamma} \frac{\partial R_{i}^{\beta}}{\partial q^{\gamma}} .
$$

We next derive the relationship between $F_{\Pi_{0}}$ and $F_{\Pi}$. Note that with respect to the given splitting

$$
\Pi_{0}: T Y=G_{0} \oplus T \mathcal{F} \cong N \mathcal{F} \oplus T \mathcal{F}
$$

any other projection $\Pi: T Y \rightarrow T Y$ can be written as the following block matrix

$$
\Pi=\left(\begin{array}{cc}
0 & 0 \\
B & I d
\end{array}\right)
$$

where $B=B_{\Pi_{0} \Pi} \circ \pi_{G_{0}}: G_{0} \rightarrow T \mathcal{F}$ is the bundle map introduced in Lemma 4.1 which is uniquely determined by $\Pi_{0}$ and $\Pi$ and vice versa. The following lemma shows their relationship in coordinates.

Lemma 4.3. Let $F_{\Pi}$ and $F_{\Pi_{0}}$ be the transverse $\Pi$-curvatures with respect to $\Pi$ and $\Pi_{0}$ respectively, and let $B=B_{\Pi_{0} \Pi}$ be the bundle map mentioned above. In terms of the foliation coordinates, we have

$$
\begin{aligned}
F_{i j}^{\beta}=F_{0, i j}^{\beta}+\left(\frac{\partial B_{j}^{\beta}}{\partial y^{2}}-\frac{\partial B_{i}^{\beta}}{\partial y^{j}}\right. & \left.+R_{i}^{\alpha} \frac{\partial B_{j}^{\beta}}{\partial q^{\alpha}}-R_{j}^{\alpha} \frac{\partial B_{i}^{\beta}}{\partial q^{\alpha}}+B_{i}^{\alpha} \frac{\partial R_{j}^{\beta}}{\partial q^{\alpha}}-B_{j}^{\alpha} \frac{\partial R_{i}^{\beta}}{\partial q^{\alpha}}\right) \\
+ & \left(B_{i}^{\alpha} \frac{\partial B_{j}^{\beta}}{\partial q^{\alpha}}-B_{j}^{\alpha} \frac{\partial B_{i}^{\beta}}{\partial q^{\alpha}}\right)
\end{aligned}
$$


Proof. Let $\left(y^{1}, \cdots, y^{\ell}, q^{1}, \cdots, q^{m-\ell}\right)$ be a foliation coordinates of $\mathcal{F}$ and let $R_{j}^{\beta}$ be the "Christoffel symbols" for $\Pi_{0}$. Let $B_{\Pi_{0} \Pi}: N \mathcal{F} \rightarrow T \mathcal{F}$ be the above bundle map associated to the pair $\Pi_{0}, \Pi$ of splittings. Using the above block decomposition of $\Pi$, it follows that the corresponding "Christoffel symbols" for $\Pi$ are given by

$$
R_{j}^{\beta}+B_{j}^{\beta}
$$

where $\left(B_{j}^{\beta}\right)$ is the matrix of $B_{\Pi_{0} \Pi}$ in terms of the bases of $N \mathcal{F}$ and $T \mathcal{F}$ associated to the foliation coordinates. Then the formula (4.9) follows immediately from (4.8) by substituting $R_{j}^{\beta}$ by $R_{j}^{\beta}+B_{j}^{\beta}$.

Now we provide an invariant description of the above formula (4.9). Consider the sheaf $\Lambda^{\bullet}\left(N^{*} \mathcal{F}\right) \otimes T \mathcal{F}$ and denote by

$$
\Omega^{\bullet}\left(N^{*} \mathcal{F} ; T \mathcal{F}\right):=\Gamma\left(\Lambda^{\bullet}\left(N^{*} \mathcal{F}\right) \otimes T \mathcal{F}\right)
$$

the group of (local) sections thereof. For an invariant interpretation of the above basis of $G_{x}$ and the transformation law (4.9), we need to use the notion of basic vector fields (or projectable vector fields) which is standard in the foliation theory (see e.g., $[\mathrm{MM}]$ ) : Consider the Lie subalgebra

$$
L(Y, \mathcal{F})=\left\{\xi \in \Gamma(T Y) \mid a d_{\xi}(\Gamma(T \mathcal{F})) \subset \Gamma(T \mathcal{F})\right\}
$$

and its quotient Lie algebra

$$
\ell(Y, \mathcal{F})=L(Y, \mathcal{F}) / \Gamma(T \mathcal{F}) .
$$

An element from $\ell(Y, \mathcal{F})$ is called a transverse vector field of $\mathcal{F}$. In general, there may not be a global basic lifting $Y$ of a given transverse vector field. But the following lemma shows that this is always possible locally.

Lemma 4.4. Let $x_{0} \in Y$ and $v \in N_{x_{0}} \mathcal{F}$. Then there exists a local basic vector field $\xi$ in a neighborhood of $x_{0}$ such that it is tangent to $G$

$$
\pi\left(\xi\left(x_{0}\right)\right)=v
$$

where $\pi: T Y \rightarrow N \mathcal{F}$ is the canonical projection.

Proof. Using the foliation coordinates, it is well-known and easy to check that $N_{x_{0}} \mathcal{F}$ is spanned by (local) basic vector fields. Let $\xi^{\prime}$ be any such transverse vector field with $\pi\left(Y^{\prime}\left(x_{0}\right)\right)=v$. Then we just take $\xi=\xi^{\prime}-\Pi\left(\xi^{\prime}\right)$ which is obviously transverse because $Y^{\prime}$ is transverse and $\Pi\left(\xi^{\prime}\right)$ is tangent to $\mathcal{F}$.

Definition 4.2. Let $\mathcal{F}$ be a foliation on $Y$. Let $\Pi \in \mathcal{A}_{E}(T Y)$ and $\Pi: T Y=$ $G_{\Pi} \oplus T \mathcal{F}$ be the $\Pi$-splitting. We call a basic vector field $\xi$ tangent to $G_{\Pi}$ a $\Pi$-basic vector field or a $G$-basic vector field.

In this point of view, the vector field

$$
Y_{i}:=\frac{\partial}{\partial y^{i}}+\sum_{\alpha=1}^{n-k} R_{i}^{\alpha} \frac{\partial}{\partial q^{\alpha}}
$$

is the unique $G$-basic vector field that satisfies

$$
Y_{j} \equiv \frac{\partial}{\partial y^{i}} \bmod T \mathcal{F},
$$

i.e., defines the same transverse vector field as $\frac{\partial}{\partial y^{2}}$. 
Definition 4.3. Let $X$ be any (local) basic vector field of $\mathcal{F}$ tangent to $G_{\Pi}$. We define the $\Pi$-Lie derivative of $B$ with respect to $X$ by the formula

$$
L_{X}^{\Pi} B=\sum_{i_{1}<\cdots<i_{\ell}} L_{X}\left(B_{i_{1} i_{2} \cdots i_{\ell}}\right) d y^{i_{1}} \wedge \cdots \wedge d y^{i_{\ell}}
$$

where $B_{i_{1} i_{2} \cdots i_{\ell}}$ is a local section of $T \mathcal{F}$ given by the local representation of $B$

$$
B=\sum_{i_{1}<\cdots<i_{\ell}} B_{i_{1} \cdots i_{\ell}} d y^{i_{1}} \wedge \cdots \wedge d y^{i_{\ell}}
$$

in any given foliation coordinates. Here $B_{i_{1} \cdots i_{\ell}}$ is the (locally defined) leafwise tangent vector field given by

$$
B_{i_{1} \cdots i_{\ell}}=B_{i_{1} \cdots i_{\ell}}^{\beta} \frac{\partial}{\partial q^{\beta}} .
$$

From now on without mentioning further, we will always assume that $B$ is locally defined, unless otherwise stated.

Definition 4.4. For any element $B \in \Gamma\left(\Lambda^{\ell}\left(N^{*} \mathcal{F}\right) ; T F\right)$, we define

$$
d^{\Pi} B \in \Gamma\left(\Lambda^{\ell+1}\left(N^{*} \mathcal{F}\right) ; T F\right)
$$

by the formula

$$
d^{\Pi} B=\sum_{j=1}^{2 k} d y^{j} \wedge L_{Y_{j}}^{\Pi} B
$$

where we call the operator $d^{\Pi}$ the $\Pi$-differential.

For given splitting $\Pi$ and a vector field $\xi$, we denote by $\xi^{\Pi}$ the projection of $\xi$ to $G=G_{\Pi}$, i.e.,

$$
\xi^{\Pi}=\xi-\Pi(\xi) .
$$

Then the definition of $d^{\Pi}$ can be also given by the same kind of formula as that of the usual exterior derivative $d$ : For given $B \in \Omega^{k}\left(N^{*} \mathcal{F} ; T \mathcal{F}\right)$ and local sections $\eta_{1}, \cdots, \eta_{k+1} \in N_{x} \mathcal{F}$, we define

$$
\begin{aligned}
d^{\Pi} B\left(v_{1},\right. & \left.\cdots, v_{k}, v_{k+1}\right) \\
& =\sum_{i}(-1)^{i-1} X_{i}\left(B\left(\eta_{1}, \cdots, \widehat{\eta}_{i}, \cdots, \eta_{k+1}\right)\right) \\
& +\sum_{i<j}(-1)^{i+j-1} B\left(\pi\left(\left[X_{i}, X_{j}\right]\right), \eta_{1}, \cdots, \widehat{\eta}_{i}, \cdots, \widehat{\eta}_{j}, \cdots, \eta_{k+1}\right)(
\end{aligned}
$$

Here $X_{i}$ is a $\Pi$-basic vector field with $\pi\left(X_{i}(x)\right)=\eta_{i}(x)$ for each given point $x \in Y$. It is straightforward to check that this definition coincides with (4.11).

Next we introduce the analog of the "bracket"

$$
[\cdot, \cdot]_{\Pi}: \Omega^{\ell_{1}}\left(N^{*} \mathcal{F} ; T \mathcal{F}\right) \otimes \Omega^{\ell_{2}}\left(N^{*} \mathcal{F} ; T \mathcal{F}\right) \rightarrow \Omega^{\ell_{1}+\ell_{2}}\left(N^{*} \mathcal{F} ; T \mathcal{F}\right) .
$$

Definition 4.5. Let $B \in \Omega^{\ell_{1}}\left(N^{*} \mathcal{F} ; T \mathcal{F}\right), C \in \Omega^{\ell_{2}}\left(N^{*} \mathcal{F} ; T \mathcal{F}\right)$. We define their bracket

$$
[B, C]_{\Pi} \in \Omega^{\ell_{1}+\ell_{2}}(N \mathcal{F} ; T \mathcal{F})
$$


by the formula

$$
\begin{aligned}
& {[B, C]_{\Pi}\left(v_{1}, \cdots, v_{\ell_{1}}, v_{\ell_{1}+1}, \cdots, v_{\ell_{1}+\ell_{2}}\right) } \\
&= \sum_{\sigma \in S_{n}} \frac{\operatorname{sign}(\sigma)}{\left(\ell_{1}+\ell_{2}\right) !}\left[B\left(X_{\sigma(1)}, \cdots, X_{\sigma\left(\ell_{1}\right)}\right), C\left(X_{\sigma\left(\ell_{1}+1\right)}, \cdots, X_{\sigma\left(\ell_{1}+\ell_{2}\right)}\right]\right. \\
&= \sum_{\tau \in S h u f f(n)} \frac{\operatorname{sign}(\tau)}{\ell_{1} ! \ell_{2} !}\left[B\left(X_{\tau(1)}, \cdots, X_{\tau\left(\ell_{1}\right)}\right),\right. \\
& C\left(X_{\tau\left(\ell_{1}+1\right)}, \cdots, X_{\tau\left(\ell_{1}+\ell_{2}\right)}\right]
\end{aligned}
$$

for each $x \in Y$ and $v_{i} \in N_{x} \mathcal{F}$, and $X_{i}$ 's are (local) ח-basic vector fields such that $\pi\left(X_{i}(x)\right)=v_{i}$ as before. Here $S_{n}$ is the symmetric group with size $n$ and Shuf $f(n) \subset S_{n}$ is the subgroup of all "shuffles". $[\cdot, \cdot]$ is the usual Lie bracket of leafwise vector fields.

For the case $\ell_{1}=\ell_{2}=1$, we derive the coordinate formula

$$
[B, C]_{\Pi}=\left(B_{i}^{\alpha} \frac{\partial C_{j}^{\beta}}{\partial q^{\alpha}}-C_{j}^{\alpha} \frac{\partial B_{i}^{\beta}}{\partial q^{\alpha}}\right) \frac{\partial}{\partial q^{\beta}} \otimes d y^{i} \wedge d y^{j} .
$$

With these definitions, we have the following "Bianchi identity" in our context.

Proposition 4.5. Let $\Pi: T Y=G \oplus T \mathcal{F}$ and $d^{\Pi}$ be the associated $\Pi$-differential. Then we have

$$
\begin{aligned}
d^{\Pi} F_{\Pi} & =0 \\
\left(d^{\Pi}\right)^{2} B & =\left[F_{\Pi}, B\right]_{\Pi} .
\end{aligned}
$$

Proof. As before, we denote $Y_{i}=\frac{\partial}{\partial y^{i}}+R_{i}^{\alpha} \frac{\partial}{\partial q^{\alpha}}$. We compute

$$
d^{\Pi} F_{\Pi}=\frac{1}{2 !} d y^{i} \wedge L_{Y_{i}}\left(F_{j k}\right) d y^{j} \wedge d y^{k}, \quad F_{j k}=F_{j k}^{\alpha} \frac{\partial}{\partial q^{\alpha}} .
$$

By definition of $F_{\Pi}$, we have

$$
L_{Y_{i}}\left(F_{j k}\right)=L_{Y_{i}}\left(\Pi\left[Y_{j}, Y_{k}\right]\right)
$$

And we have

$$
L_{Y_{i}}\left(\Pi\left[Y_{j}, Y_{k}\right]\right)=L_{Y_{i}} \Pi\left(\left[Y_{j}, Y_{k}\right]\right)+\Pi\left(\left[Y_{i},\left[Y_{j}, Y_{k}\right]\right]\right) .
$$

Here after taking the cyclic sum over $i, j, k$, the second term vanishes by the Jacobi identity of the Lie bracket. On the other hand, differentiating $\Pi^{2}=\Pi$, we derive

$$
\left(L_{X} \Pi\right)([Y, Z])=\Pi\left(L_{X} \Pi\right)([Y, Z])+\left(L_{X} \Pi \cdot \Pi\right)([Y, Z])
$$

in general. Furthermore, if we restrict to the basic vector fields $X, Y, Z$ and so $[Y, Z]$ is also basic, then it immediately follows from $\Pi^{2}=\Pi$ that the second term vanishes. The first term becomes

$$
\begin{aligned}
\Pi\left(L_{X} \Pi\right)([Y, Z]) & =\Pi\left(L_{X}(\Pi[Y, Z])-\Pi \cdot \Pi L_{X}([Y, Z])\right. \\
& =\Pi([X, \Pi[Y, Z]]-[X,[Y, Z]])=-\Pi([X,[Y, Z]-\Pi[Y, Z]) .
\end{aligned}
$$

When this identity is applied to

$$
X=Y_{i}, Y=Y_{j}, Z=Y_{k},
$$


this term also vanishes because we have

$$
\left[Y_{j}, Y_{k}\right]=\left[\frac{\partial}{\partial y^{j}}+R_{j}^{\alpha} \frac{\partial}{\partial q^{\alpha}}, \frac{\partial}{\partial y^{k}}+R_{k}^{\alpha} \frac{\partial}{\partial q^{\alpha}}\right]=F_{j k}^{\alpha} \frac{\partial}{\partial q^{\alpha}}
$$

which is tangent to $\mathcal{F}$. This finishes proof of the Bianchi identity, $d^{\Pi} F_{\Pi}=0$.

For the proof of (4.16), we consider two П-basic vector fields

$$
Y_{i}=\frac{\partial}{\partial y^{j}}+R_{i}^{\alpha} \frac{\partial}{\partial q^{\alpha}}, Y_{j}=\frac{\partial}{\partial y^{j}}+R_{j}^{\alpha} \frac{\partial}{\partial q^{\alpha}} .
$$

We recall

$$
d^{\Pi} B=d y^{j} \wedge L_{Y_{j}} B=\frac{1}{\ell !} L_{Y_{j}}\left(B_{i_{1} \cdots i_{\ell}}\right) d y^{j} \wedge d y^{i_{1}} \wedge \cdots \wedge d y^{i_{\ell}}
$$

Therefore we have

$$
\begin{aligned}
\left(d^{\Pi}\right)^{2} B & =\frac{1}{\ell !} L_{Y_{i}} L_{Y_{j}}\left(B_{i_{1} \cdots i_{\ell}}\right) d y^{i} \wedge d y^{j} \wedge d y^{i_{1}} \wedge \cdots \wedge d y^{i_{\ell}} \\
& =\sum_{i<j} \frac{1}{\ell !}\left(L_{Y_{i}} L_{Y_{j}}-L_{Y_{j}} L_{Y_{i}}\right)\left(B_{i_{1} \cdots i_{\ell}}\right) d y^{i} \wedge d y^{j} \wedge d y^{i_{1}} \wedge \cdots \wedge d y^{i_{\ell}} \\
& =\sum_{i<j} \frac{1}{\ell !} L_{\left[Y_{i}, Y_{j}\right]}\left(B_{i_{1} \cdots i_{\ell}}\right) d y^{i} \wedge d y^{j} \wedge d y^{i_{1}} \wedge \cdots \wedge d y^{i_{\ell}}
\end{aligned}
$$

Here we note that $\left[Y_{i}, Y_{j}\right]$ is tangent to $\mathcal{F}$ and hence

$$
\left[Y_{i}, Y_{j}\right](x)=\Pi\left[Y_{i}, Y_{j}\right](x)=F_{\Pi}\left(u_{i}, u_{j}\right)
$$

where $u_{i}=\pi\left(Y_{i}\right)(x)$. Therefore we have

$$
\left(d^{\Pi}\right)^{2} B=\left[F_{\Pi}, B\right]_{\Pi}
$$

on $\Omega^{\ell_{1}+\ell_{2}}\left(N^{*} \mathcal{F} ; T \mathcal{F}\right)$ which finishes the proof of (4.16).

Combining the above discussion, the transformation law (4.9) in coordinates is translated into the following invariant form.

Proposition 4.6. Let $\Pi, \Pi_{0}$ be two splittings as in Lemma 4.3 and $B_{\Pi_{0} \Pi \in} \in$ $\Gamma\left(N^{*} \mathcal{F} \otimes T \mathcal{F}\right)$ be the associated section. Then we have

$$
F_{\Pi}=F_{\Pi_{0}}+d^{\Pi_{0}} B_{\Pi_{0} \Pi}+\left[B_{\Pi_{0} \Pi}, B_{\Pi_{0} \Pi}\right]_{\Pi_{0}} .
$$

Remark 4.6. We would like to emphasize that the bracket $[\cdot, \cdot]_{\Pi}$ we defined is not bilinear over $C^{\infty}(Y)$, but linear over the so-called subalgebra of basic functions: a smooth function $f: Y \rightarrow \mathbb{R}$ is called basic, if it is constant along the leaves(see [MM], [To] for the definition). It seems that a good formulation of invariant objects "over the leaf space" should be in terms of the Haefliger-type cocycles and germs of $\Omega^{\bullet}(T ; T \mathcal{F})$ over transverse sections $T$ of the foliation $\mathcal{F}$ as in [Ha] for his definition of $\Omega^{\bullet}(\operatorname{Tr} \mathcal{F})$. We postpone elsewhere a full disclosure of geometric structures that arise in the study of the deformation problem of foliations $\mathcal{F}$ and the role of the transverse curvature $F_{\Pi}$. 


\section{GeOmetry of the NULl FOliation}

In this section, we will apply the leaf space calculus developed in the previous section to the null foliation. Let $(Y, \omega)$ be a pre-symplectic manifold and denote by $\mathcal{F}$ the associated null foliation. About the range of indices of $i$ and $\alpha$ from section 4 we use

$$
m=n+k, \ell=2 k, \quad 1 \leq i \leq 2 k, 1 \leq \alpha \leq n-k
$$

for the null foliation for $(Y, \omega)$.

Using Lemma 4.1 we now state a uniqueness statement in the symplectic thickening of $(Y, \omega)$. This precise form of the neighborhood theorem will be a crucial ingredient for our proof in section 10 of the gauge equivalence of strong homotopy Lie algebroids that we associate to the splittings $\Pi$. We denote by $\omega_{\Pi}$ the symplectic form given in (3.3) associated to the splitting $\Pi$.

Proposition 5.1. For given two splittings $\Pi_{0}$, $\Pi$, there exist neighborhoods $U, U^{\prime}$ of the zero section $Y \subset E^{*}$ and a diffeomorphism $\phi: U \rightarrow U^{\prime}$ such that

(1) $\phi^{*} \omega_{\Pi}=\omega_{\Pi_{0}}$,

(2) $\left.\phi\right|_{Y} \equiv i d$, and $\left.T \phi\right|_{T_{Y} E^{*}} \equiv i d$ where $T_{Y} E^{*}$ is the restriction of $T E^{*}$ to $Y$.

Proof. Since $\mathcal{A}_{E}(T Y)$ is contractible, we can choose a smooth family

$$
\left\{\Pi_{t}\right\}_{0 \leq t \leq 1}, \quad \Pi_{0}=\Pi_{0}, \Pi_{1}=\Pi .
$$

Denoting $\omega_{t}:=\omega_{\Pi_{t}}$, we have

$$
\omega_{t}-\omega_{0}=d\left(\theta_{0}-\theta_{t}\right)
$$

$\theta_{t}$ is the one-form $\theta_{G}$ associated to $\Pi_{t}$ as defined in 6.4 . From the definition, it follows $\left.\theta_{t}\right|_{T_{Y} E^{*}} \equiv 0$ and hence

$$
\left.\left(\theta_{0}-\theta_{t}\right)\right|_{T_{Y} E^{*}} \equiv 0
$$

for all $0 \leq t \leq 1$. With these, the proof follows from the standard Moser's homotopy method [We1], since $\omega_{t}$ are all nondegenerate and homologous to each other in a sufficiently small neighborhood of the zero section $Y \subset E^{*}$.

For the study of the deformation problem of pre-symplectic structures it is crucial to understand the transverse geometry of the null foliation. First we note that the pre-symplectic form $\omega$ carries a natural transverse symplectic form. This defines the symplectic analog to the much-studied Riemannian foliation (see [Mo], [To] for example).

Proposition 5.2. Let $\mathcal{F}$ be the null foliation of the pre-symplectic manifold $(Y, \omega)$. Then the pre-symplectic form $\omega$ defines a transverse symplectic form on $\mathcal{F}$ in the following sense:

(1) $\operatorname{ker}\left(\omega_{x}\right)=T_{x} \mathcal{F}$ for any $x \in M$, and

(2) $L_{X} \omega=0$ for any vector field on $M$ tangent to $\mathcal{F}$

Proof. The first statement is trivial by definition of the null foliation and the second is an immediate consequence of the Cartan identity

$$
\left.\left.L_{X} \omega=d(X\rfloor \omega\right)+X\right\rfloor d \omega .
$$

The second term on the right hand side vanishes since $\omega$ is closed, and the first also vanishes if $X$ is tangent to the null foliation $\mathcal{F}$. 
One immediate consequence of the presence of the transverse symplectic form, together with the fact that the pre-symplectic form $\omega$ is closed, is that any transverse section $T$ of the foliation $\mathcal{F}$ carries a natural symplectic form: in any foliation coordinates, it follows from $E=\operatorname{ker} \omega=\operatorname{span}\left\{\frac{\partial}{\partial q^{\alpha}}\right\}_{1 \leq \alpha \leq n-k}$ that we have

$$
\pi^{*} \omega=\frac{1}{2} \omega_{i j} d y^{i} \wedge d y^{j}
$$

where $\omega_{i j}=\omega\left(\frac{\partial}{\partial y^{2}}, \frac{\partial}{\partial y^{3}}\right)$ is skew-symmetric and invertible. And closedness of $\omega$ implies that $\omega_{i j}$ is independent of $q^{\alpha}$ 's. Note that this expression is independent of the choice of splitting as long as $y^{1}, \cdots, y^{2 k}$ are those coordinates that characterize the leaves of $E$ by (4.3).

The proof of the following proposition is straightforward by definition of the holonomy map and is omitted (see [Proposition 2.5, MM] for a proof of its Riemannian analog).

Proposition 5.3. Let $L$ be a leaf of the null foliation $\mathcal{F}$ on $(Y, \omega), \lambda$ a path in $L$, and let $T$ and $S$ be transverse sections of $\mathcal{F}$ with $\lambda(0) \in T$ and $\lambda(1) \in S$. Then the holonomy map

$$
h o l^{S, T}(\lambda):(T, \lambda(0)) \rightarrow(S, \lambda(1))
$$

defines the germ of a symplectic diffeomorphism.

Now for the case of the null foliation $\mathcal{F}$ which carries a transverse symplectic structure, we can go one step further with the curvature $F_{\Pi}$ from the case of general foliations: we can do the operation of "raising indices" using symplectic form like a metric. We recall that $\omega$ defines a non-degenerate bilinear form on $N \mathcal{F}$. We denote by $\omega^{-1}$ the natural bilinear form induced on $N^{*} \mathcal{F}$. We refer to section 9 for more on this.

Now we introduce the following notion of symplectic mean transverse $\Pi$-curvature of the null foliation $\mathcal{F}$. This generalizes the Reeb vector field of a contact form on the contact manifold $(Y, \xi)$ to arbitrary pre-symplectic manifolds.

Definition 5.1. Define the section $\rho_{\Pi} \in \Gamma(T \mathcal{F})$ by

$$
\rho_{\Pi}=\frac{1}{2 k} \operatorname{trace}_{\omega} F_{\Pi}:=\frac{1}{2 k}\left\langle F_{\Pi}, \omega^{-1}\right\rangle
$$

where $\omega^{-1}$ is the inverse of $\omega$ on $N \mathcal{F}$ and $\langle\cdot, \cdot\rangle$ is the natural pairing between $\Lambda^{2} G^{*}$ and $\Lambda^{2}(N \mathcal{F})$. In a foliation coordinates, it is given by

$$
\rho_{\Pi}=\frac{1}{2 k} F_{i j}^{\beta} \omega^{i j} \frac{\partial}{\partial q^{\beta}}
$$

where $\left(\omega^{i j}\right)$ is the inverse of $\left(\omega_{i j}\right)$ with $\omega=\frac{1}{2} \omega_{i j} d y^{i} \wedge d y^{j}$ (see section 6 ).

Theorem 5.4. Let $(Y, \xi)$ be a contact manifold of dimension $2 n-1$ and with $\xi$ its contact distribution. Choose a contact one-form $\theta$ and consider the pre-symplectic form $\omega=-d \theta$. Denote by $X_{\theta}$ the associated Reeb vector field. We set $E=\operatorname{ker} d \theta$ and $\mathcal{F}_{\theta}$ be the associated foliation (or the line field). Consider the projection $\Pi$ : $T Y \rightarrow T Y$ such that

$$
G_{\Pi}=\xi
$$

Then for any $X, Y \in \xi \cong N \mathcal{F}_{\theta}$, we have

$$
F_{\Pi}(X, Y)=\theta([X, Y]) X_{\theta}=-d \theta(X, Y) \cdot X_{\theta}
$$


and

$$
\rho_{\Pi}=X_{\theta} .
$$

Proof. Recall that the contact vector field $X_{\theta}$ is the unique vector field that satisfies

$$
\left.\left.X_{\theta}\right\rfloor \theta=1, \quad X_{\theta}\right\rfloor d \theta=0 .
$$

Therefore it immediately implies

$$
F_{\Pi}(X, Y)=\Pi([X, Y])=\theta([X, Y]) X_{\theta}
$$

for any $X, Y \in \xi$ since we have $\theta\left([X, Y]-\theta([X, Y]) X_{\theta}\right)=0$, i.e,

$$
[X, Y]-\theta([X, Y]) X_{\theta} \in \xi=G_{\Pi} .
$$

The second identity of (5.4) follows from the definition of the exterior derivative $d \theta$ and the defining equation of contact form $\xi=\operatorname{ker} \theta$.

The identity (5.5) immediately follows from the choice of pre-symplectic form $\omega=-d \theta$ and (5.4).

The following is an interesting consequence of this theorem.

Corollary 5.5. Let $\left(X, \omega_{X}\right)$ be a given symplectic manifold and $J$ be a compatible almost complex structure. Denote by $\mathcal{H}$ yper $\left(X, \omega_{X}, J\right)$ the space of hypersurfaces with the induced pre-symplectic form $\omega$ and with the Riemannian metric induced from $g_{J}=\omega_{X}(\cdot, J \cdot)$. We choose the orthogonal splitting $\Pi: T Y=G \oplus T \mathcal{F}$ for each hypersurface $Y \in \mathcal{H} y p e r\left(X, \omega_{X}, J\right)$ and denote by $\rho_{(J, Y)}$ the corresponding symplectic mean transverse curvature. Let $\operatorname{Cont}\left(X, \omega_{X}, J\right)$ the subset of contact hypersurface with the contact form $\theta_{(J, Y)}:=\omega_{X}\left(N_{J}, \cdot\right)$ where $N_{J}$ is the unit (positive) normal vector field and denote by $X_{(J, Y)}$ the Reeb vector field of $\theta_{(J, Y)}$. Then the assignment

$$
(J, Y) \in \mathcal{J}(X, \omega) \times \mathcal{H} y p e r\left(X, \omega_{X}, J\right) \mapsto \rho_{(J, Y)}
$$

is continuous with respect to the $C^{\infty}$-topology which extends the assignment of the Reeb vector fields

$$
(J, Y) \in \mathcal{J}(X, \omega) \times \mathcal{C} \text { ont }\left(X, \omega_{X}, J\right) \mapsto X_{(J, Y)} .
$$

We believe that this corollary will play some role in the study of Hamiltonian dynamics on the hypersurfaces. This will be a subject of the future study. We also refer to [Oh] for a study of coisotropic submanifolds in Kähler manifolds with respect to the canonical orthogonal splitting $T Y=N_{J} \mathcal{F} \oplus T \mathcal{F}$ where $N_{J} \mathcal{F}$ is the normal bundle of $\mathcal{F}$ with respect to the Kähler metric $g_{J}=\omega(\cdot, J \cdot)$.

Another important geometric structure related to the bundle

$$
T \mathcal{F}=E=\operatorname{ker} \omega \rightarrow Y
$$

is the structure of a Lie algebroid (see e.g. [CW], [MM], [NT] for its definition) : the anchor map $E \rightarrow T Y$ is just the inclusion map and the Lie bracket on the sections of $E$ is just the usual bracket of vector fields on $Y$. This induces the leafwise differential $d_{\mathcal{F}}$ and defines the leafwise de Rham complex $\Omega^{*}(\mathcal{F})$, which will play an important role in the deformation problem of pre-symplectic structures and coisotropic submanifolds later. 


\section{Geometry of the Symplectic thickening}

In this section and the next two, we will unravel the geometric and algebraic structure that governs the deformation problem of coisotropic submanifolds up to Hamiltonian isotopy. As we showed in section 2, it is enough to study this in the model space $\left(U, \omega_{U}\right)$ constructed in section 2. Precise formulation of the problem is in order.

Again we start with a splitting

$$
T Y=G \oplus E,
$$

the associated bundle projection $\Pi: T Y \rightarrow T Y$, the associated canonical one form $\theta_{G}$, and the symplectic form

$$
\omega_{E^{*}}=\pi^{*} \omega-d \theta_{G}
$$

on $U \subset E^{*}$. Note the projection map $\pi: U \rightarrow Y$ induces a foliation $\pi^{-1}(\mathcal{F})$ on $U$ in a canonical way. The leaves of $\pi^{-1}(\mathcal{F})$ are the preimages of the leaves of $\mathcal{F}$, which are symplectic submanifolds of $U$. When we choose foliation coordinates $\left(y^{1}, \cdots, y^{2 k}, q^{1}, \cdots, q^{n-k}\right)$ on $Y$, we can extend these coordinates to foliation coordinates of $\pi^{-1}(\mathcal{F})$

$$
\left(y^{1}, \cdots, y^{2 k}, q^{1}, \cdots, q^{n-k}, p_{1}, \cdots, p_{n-k}\right)
$$

so that

$$
y^{1}=c^{1}, \cdots, y^{2 k}=c^{2 k}, \quad c^{i} \text { s constant }
$$

defines the leaves of the foliation $\pi^{-1}(\mathcal{F})$. We will construct these coordinates explicitly below. We have the following commutative diagram of exact sequences

$$
\begin{aligned}
& 0 \rightarrow T \pi^{-1}(\mathcal{F}) \quad \rightarrow \quad T U \rightarrow N\left(\pi^{-1}(\mathcal{F})\right) \rightarrow 0
\end{aligned}
$$

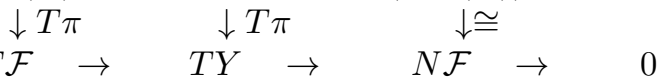

Note that for a given splitting $\Pi: T Y=G \oplus T \mathcal{F}$, due to the presence of the symplectic form $\omega_{U}$, there exists the unique splitting of $T U$

$$
T U=G^{\sharp} \oplus T \pi^{-1}(\mathcal{F})
$$

that satisfies

$$
G^{\sharp}=\left(T_{\alpha} \pi^{-1}(\mathcal{F})\right)^{\omega_{U}}
$$

for any $\alpha \in U$, which is invariant under the action of symplectic diffeomorphisms on $\left(U, \omega_{U}\right)$ that preserve the leaves of $\pi^{-1}(\mathcal{F})$.

Definition 6.1. We call the above unique splitting the leafwise symplectic connection of $U \rightarrow Y$ compatible to the splitting $\Pi: T Y=G \oplus T \mathcal{F}$ or simply a leafwise symplectic $\Pi$-connection of $U \rightarrow Y$.

We would like to emphasize that this connection is not a vector bundle connection of $E^{*}$ although $U$ is a subset of $E^{*}$, which reflects nonlinearity of this connection.

Note that the splitting $\Pi$ naturally induces the splitting

$$
\Pi_{*}: T^{*} Y=(T \mathcal{F})^{\circ} \oplus G^{\circ}
$$

where $(T \mathcal{F})^{\circ}$ and $G^{\circ}$ are the annihilators of $T \mathcal{F}$ and $G$ respectively. We denote by $\Pi^{\sharp}: T U \rightarrow T U$ the projection to $T\left(\pi^{-1} \mathcal{F}\right)$ associated to 6.2 . We now derive the coordinate expression of the leafwise symplectic $\Pi$-connection. Let

$$
\left(y^{1}, \cdots, y^{2 k}, y^{2 k+1}, \cdots, y^{n+k}\right)
$$


be coordinates on $Y$ adapted to the null foliation on an open subset $V \subset Y$ as before. By choosing the frame

$$
\left\{f_{1}^{*}, \cdots, f_{n-k}^{*}\right\}
$$

of $E^{*}$ that is dual to the frame $\left\{\frac{\partial}{\partial q^{1}}, \cdots, \frac{\partial}{\partial q^{n-k}}\right\}$ of $E$, we introduce the canonical coordinates on $E^{*}$ by writing an element $\alpha \in E^{*}$ as a linear combination of $\left\{f_{1}^{*}, \cdots, f_{n-k}^{*}\right\}$

$$
\alpha=p_{\beta} f_{\beta}^{*},
$$

and taking

$$
\left(y^{1}, \cdots, y^{2 k}, q^{1}, \cdots, q^{n-k}, p_{1}, \cdots, p_{n-k}\right)
$$

as the associated coordinates. To derive the coordinate expression of $\theta_{G}$, we compute

$$
\begin{aligned}
\theta_{G}\left(\frac{\partial}{\partial y^{i}}\right) & =\alpha\left(p_{G} \circ T \pi\left(\frac{\partial}{\partial y^{i}}\right)\right)=\alpha\left(p_{G}\left(\frac{\partial}{\partial y^{i}}\right)\right) \\
& =p_{\beta} f_{\beta}^{*}\left(-R_{i}^{\alpha} \frac{\partial}{\partial q^{\alpha}}\right)=-p_{\alpha} R_{i}^{\alpha}, \\
\theta_{G}\left(\frac{\partial}{\partial q^{\beta}}\right) & =p_{\beta}, \quad \theta_{G}\left(\frac{\partial}{\partial p_{\beta}}\right)=0 .
\end{aligned}
$$

Hence we derive

$$
\theta_{G}=p_{\beta}\left(d q^{\beta}-R_{i}^{\beta} d y^{i}\right)
$$

Here we note that

$$
\left.\left(d q^{\beta}-R_{i}^{\beta} d y^{i}\right)\right|_{G_{x}} \equiv 0 .
$$

This shows that if we identify $E^{*}=T^{*} \mathcal{F}$ with

$$
G^{\circ}:=\left.\operatorname{im}(\Pi)^{*}\right|_{E^{*}} \subset T^{*} Y
$$

via the embedding $\left.(\Pi)^{*}\right|_{E^{*}}: E^{*} \hookrightarrow T^{*} Y$ induced by the splitting $\Pi: T Y=G \oplus T \mathcal{F}$, then we may write the dual frame on $T^{*} \mathcal{F}$ as

$$
f_{\beta}^{*}=d q^{\beta}-R_{i}^{\beta} d y^{i} .
$$

Motivated by this, we write

$$
\begin{aligned}
d \theta_{G}= & d p_{\beta} \wedge\left(d q^{\beta}-R_{i}^{\beta} d y^{i}\right)-p_{\beta} d R_{i}^{\beta} \wedge d y^{i} \\
= & d p_{\beta} \wedge\left(d q^{\beta}-R_{i}^{\beta} d y^{i}\right)-p_{\beta} \frac{\partial R_{i}^{\beta}}{\partial y^{j}} d y^{j} \wedge d y^{i} \\
& -p_{\beta} \frac{\partial R_{i}^{\beta}}{\partial q^{\gamma}} d q^{\gamma} \wedge d y^{i} .
\end{aligned}
$$


Combining (6.6) and (5.1), we have

$$
\begin{aligned}
\omega_{U}= & \pi^{*} \omega-d \theta_{G} \\
= & \left(\frac{1}{2} \omega_{i j}-p_{\beta} \frac{\partial R_{j}^{\beta}}{\partial y^{i}}\right) d y^{i} \wedge d y^{j}-d p_{\delta} \wedge\left(d q^{\delta}-R_{i}^{\delta} d y^{i}\right) \\
& +p_{\nu} \frac{\partial R_{i}^{\nu}}{\partial q^{\gamma}} d q^{\gamma} \wedge d y^{i} \\
= & \left(\frac{1}{2} \omega_{i j}-p_{\beta} \frac{\partial R_{j}^{\beta}}{\partial y^{i}}-p_{\beta} \frac{\partial R_{i}^{\beta}}{\partial q^{\gamma}} R_{j}^{\gamma}\right) d y^{i} \wedge d y^{j} \\
& -\left(d p_{\delta}+p_{\beta} \frac{\partial R_{i}^{\beta}}{\partial q^{\delta}} d y^{i}\right) \wedge\left(d q^{\delta}-R_{j}^{\delta} d y^{j}\right) \\
= & \left(\frac{1}{2} \omega_{i j}-p_{\beta}\left(\frac{\partial R_{j}^{\beta}}{\partial y^{i}}-\frac{\partial R_{i}^{\beta}}{\partial q^{\gamma}} R_{j}^{\gamma}\right)\right) d y^{i} \wedge d y^{j} \\
& -\left(d p_{\beta}+p_{\beta} \frac{\partial R_{i}^{\beta}}{\partial q^{\gamma}} d y^{i}\right) \wedge\left(d q^{\delta}-R_{j}^{\delta} d y^{j}\right) \\
= & \frac{1}{2}\left(\omega_{i j}-p_{\beta} F_{i j}^{\beta}\right) d y^{i} \wedge d y^{j} \\
& -\left(d p_{\delta}+p_{\beta} \frac{\partial R_{i}^{\beta}}{\partial q^{\delta}} d y^{i}\right) \wedge\left(d q^{\delta}-R_{j}^{\delta} d y^{j}\right)
\end{aligned}
$$

where $F_{i j}^{\beta}$ are the components of the transverse $\Pi$-curvature of the null-foliation. Here the last identity comes from the skew-symmetry of $\omega_{i j}$ and by anti-symmetrizing the first term of the identity right before.

Note that we have

$$
T \pi^{-1}(\mathcal{F})=\operatorname{span}\left\{\frac{\partial}{\partial q^{1}}, \cdots, \frac{\partial}{\partial q^{n-k}}, \frac{\partial}{\partial p_{1}}, \cdots, \frac{\partial}{\partial p_{n-k}}\right\}
$$

which is independent of the choice of the above induced foliation coordinates of $T U$.

Now we compute $\left(T \pi^{-1}(\mathcal{F})\right)^{\omega_{U}}$ in $T U$ in terms of these induced foliation coordinates. We will determine when the expression

satisfies

$$
a^{j}\left(\frac{\partial}{\partial y^{j}}+R_{j}^{\alpha} \frac{\partial}{\partial q^{\alpha}}\right)+b^{\beta} \frac{\partial}{\partial q^{\beta}}+c_{\gamma} \frac{\partial}{\partial p_{\gamma}}
$$

$$
\omega_{U}\left(a^{j}\left(\frac{\partial}{\partial y^{j}}+R_{j}^{\alpha} \frac{\partial}{\partial q^{\alpha}}\right)+b^{\beta} \frac{\partial}{\partial q^{\beta}}+c_{\gamma} \frac{\partial}{\partial p_{\gamma}}, T \pi^{-1}(\mathcal{F})\right)=0 .
$$

It is immediate to see by pairing with $\frac{\partial}{\partial p_{\mu}}$

$$
b^{\beta}=0, \quad \beta=1, \cdots, n-k .
$$

Next we study the equation

$$
0=\omega_{U}\left(a^{j}\left(\frac{\partial}{\partial y^{j}}+R_{j}^{\alpha} \frac{\partial}{\partial q^{\alpha}}\right)+c_{\gamma} \frac{\partial}{\partial p_{\gamma}}, \frac{\partial}{\partial q^{\nu}}\right)
$$

for all $\nu=1, \cdots, n-k$. A straightforward check provides

$$
-a^{j} p_{\beta} \frac{\partial R_{j}^{\beta}}{\partial q^{\nu}}-c_{\nu}=0
$$


for all $\nu$ and $j$. Combining (6.9) and (6.10), we have obtained

$$
\left(T \pi^{-1}(\mathcal{F})\right)^{\omega_{U}}=\operatorname{span}\left\{\frac{\partial}{\partial y^{j}}+R_{j}^{\alpha} \frac{\partial}{\partial q^{\alpha}}-p_{\beta} \frac{\partial R_{j}^{\beta}}{\partial q^{\nu}} \frac{\partial}{\partial p_{\nu}}\right\}_{1 \leq j \leq 2 k} .
$$

Remark 6.2. Just as we have been considering $\Pi: T Y=G \oplus T \mathcal{F}$ as a "connection" over the leaf space, we may consider the splitting $\Pi^{\sharp}: T U=G^{\sharp} \oplus T\left(\pi^{-1} \mathcal{F}\right)$ as the leaf space connection canonically induced from $\Pi$ under the fiber-preserving map

$$
\pi: U \rightarrow Y
$$

over the same leaf space $Y / \sim$ : Note that the space of leaves of $\mathcal{F}$ and $\pi^{-1} \mathcal{F}$ are canonically homeomorphic.

\section{Master Equation in COORDinates}

We will now study the condition that the graph of a section $s: Y \rightarrow E^{*} \cong N Y$ is coisotropic with respect to $\omega_{U}$. We call the corresponding equation the classical part of the master equation. We study the full (local) moduli problem of coisotropic submanifolds by analyzing the condition that the graph of a section $s: Y \rightarrow U$ in the symplectic thickening $U$ is to be coisotropic with respect to $\omega_{U}$.

Recall that an Ehresmann connection of $U \rightarrow Y$ with a structure group $H$ is a splitting of the exact sequence

$$
0 \rightarrow V T U \longrightarrow T U \stackrel{T \pi}{\longrightarrow} T Y \rightarrow 0
$$

that is invariant under the action of the group $H$. Here $H$ is not necessarily a finite dimensional Lie group. In other words, an Ehresmann connection is a choice of decomposition

$$
T U=H T U \oplus V T U
$$

that is invariant under the fiberwise action of $H$. Recalling that there is a canonical identification $V_{\alpha} T U \cong V_{\alpha} T E^{*} \cong E_{\pi(\alpha)}^{*}$, a connection can be described as a horizontal lifting $H T_{\alpha} U$ of $T Y$ to $T U$ at each point $y \in Y$ and $\alpha \in U \subset E^{*}$ with $\pi(\alpha)=y$. We denote by $F^{\#} \subset H T U$ the horizontal lifting of a subbundle $F \subset T Y$ in general.

Let $\left(y^{1}, \cdots, y^{2 k}, q^{1}, \cdots, q^{n-k}\right)$ be a foliation coordinates of $\mathcal{F}$ on $Y$ and

$$
\left(y^{1}, \cdots, y^{2 k}, q^{1}, \cdots, q^{n-k}, p_{1}, \cdots, p_{n-k}\right)
$$

be the induced foliation coordinates of $\pi^{-1}(\mathcal{F})$ on $U$. Then $G^{\#}=\left(T \pi^{-1}(\mathcal{F})\right)^{\omega_{U}}$ has the natural basis given by

$$
e_{j}=\frac{\partial}{\partial y^{j}}+R_{j}^{\alpha} \frac{\partial}{\partial q^{\alpha}}-p_{\beta} \frac{\partial R_{j}^{\beta}}{\partial q^{\nu}} \frac{\partial}{\partial p_{\nu}}
$$

which are basic vector fields of $T\left(\pi^{-1} \mathcal{F}\right)$. We also denote

$$
f_{\alpha}=\frac{\partial}{\partial q^{\alpha}}
$$

We define a local lifting of $E$

$$
E^{\sharp}=\operatorname{span}\left\{f_{1}, \cdots, f_{n-k}\right\} .
$$


Remark 7.1. (1) When a splitting $\Pi: T Y=G \oplus T \mathcal{F}$ is given, $G^{\sharp}$ is canonically defined which is independent of the foliation coordinates. Unlike $G^{\sharp}$, $E^{\sharp}$ depends on the choice of foliation coordinates. However for another choice of foliation coordinates $\left(\underline{y}^{1}, \cdots, \underline{y}^{2 k}, \underline{q}^{1}, \cdots, \underline{q}^{n-k}\right), E^{\sharp}$ will have a basis in the form

$$
\frac{\partial}{\partial \underline{q}^{\alpha}}+A_{\beta}^{\alpha} \frac{\partial}{\partial \underline{p}_{\beta}}
$$

for $\alpha=1, \cdots, n-k$, where the matrix $\left(A_{\beta}^{\alpha}\right)$ is symmetric. The latter follows from the fact that $E^{\sharp}$ is isotropic.

(2) The choice of splitting

$$
T\left(\pi^{-1} \mathcal{F}\right)=E^{\sharp} \oplus V T U
$$

may be considered as the analog of $\mathcal{F}$-partial connection associated to the principal $G$-bundle $P \rightarrow B$ for a Lie groupoid whose associated Lie algebroid is the one associated to the null foliation $\mathcal{F}$, except that in our case we do not have associated the global Lie groupoid in sight. We refer to [section $5, \mathrm{MM}]$ for the precise definition of principal $G$-bundles of a Lie groupoid $G$ and the $\mathcal{F}$-partial connection. The relevant groupoid-like object in our case is the symplectic thickening $\pi: U \rightarrow Y$ which can be interpreted as the "integrated" object of our strong homotopy Lie algebroid that we introduce in the next section. We hope to have more detailed discussion elsewhere.

(7.1) provides a local splitting

$$
T U=\left(G^{\sharp} \oplus E^{\sharp}\right) \oplus V T U \rightarrow U
$$

and defines a locally defined Ehresmann connection where $V T U$ is the vertical tangent bundle of $T U$. From the expression (6.8) of $\omega_{U}$, it follows that $G^{\sharp} \oplus E^{\sharp}$ is a coisotropic lifting of $T Y$ to $T U$. We denote by $\Pi^{v}: T U \rightarrow V T U$ the vertical projection with respect to this splitting.

With this preparation, we are finally ready to derive the master equation. Let $s: Y \rightarrow U \subset E^{*}$ be a section and denote

$$
\nabla s:=\Pi^{v} \circ d s
$$

its locally defined "covariant derivative". In coordinates $\left(y^{1}, \cdots, y^{2 k}, q^{1}, \cdots, q^{n-k}\right)$, we have

$$
\begin{aligned}
d s\left(\frac{\partial}{\partial y^{j}}\right) & =\frac{\partial}{\partial y^{j}}+\frac{\partial s_{\alpha}}{\partial y^{j}} \frac{\partial}{\partial p_{\alpha}} \\
& =e_{j}-R_{j}^{\alpha} \frac{\partial}{\partial q^{\alpha}}+s_{\beta} \frac{\partial R_{j}^{\beta}}{\partial q^{\nu}} \frac{\partial}{\partial p_{\nu}}+\frac{\partial s_{\alpha}}{\partial y^{j}} \frac{\partial}{\partial p_{\alpha}} \\
& =e_{j}-R_{j}^{\alpha} f_{\alpha}+\left(s_{\beta} \frac{\partial R_{j}^{\beta}}{\partial q^{\nu}} \frac{\partial}{\partial p_{\nu}}+\frac{\partial s_{\alpha}}{\partial y^{j}} \frac{\partial}{\partial p_{\alpha}}\right) .
\end{aligned}
$$

Therefore we have derived

$$
\nabla s\left(\frac{\partial}{\partial y^{j}}\right)=\left(\frac{\partial s_{\nu}}{\partial y^{j}}+s_{\beta} \frac{\partial R_{j}^{\beta}}{\partial q^{\nu}}\right) \frac{\partial}{\partial p_{\nu}} .
$$


Similarly we compute

$$
\begin{aligned}
d s\left(\frac{\partial}{\partial q^{\nu}}\right) & =\frac{\partial}{\partial q^{\nu}}+\frac{\partial s_{\alpha}}{\partial q^{\nu}} \frac{\partial}{\partial p_{\alpha}} \\
& =\frac{\partial}{\partial q^{\nu}}+\frac{\partial s_{\alpha}}{\partial q^{\nu}} \frac{\partial}{\partial p_{\alpha}}
\end{aligned}
$$

and so

$$
\nabla s\left(\frac{\partial}{\partial q^{\nu}}\right)=\frac{\partial s_{\alpha}}{\partial q^{\nu}} \frac{\partial}{\partial p_{\alpha}} .
$$

Recalling that $T_{\alpha} U=\left(E_{\alpha}^{\#} \oplus V T_{\alpha} U\right)^{\omega_{U}} \oplus E_{\alpha}^{\#} \oplus V T_{\alpha} U$, we conclude that the graph of $d s$ with respect to the frame

$$
\left\{e_{1}, \cdots, e_{2 k}, f_{1}, \cdots, f_{n-k}, \frac{\partial}{\partial p_{1}}, \cdots, \frac{\partial}{\partial p_{n-k}}\right\}
$$

can be expressed by the linear map

$$
\begin{aligned}
A_{H}:\left(E^{\#} \oplus V T U\right)^{\omega_{U}} & \rightarrow V T U \cong E^{*} ; \quad\left(A_{H}\right)_{\alpha}^{i}=\nabla_{i} s_{\alpha}, \\
A_{I}: E^{\#} & \rightarrow V T U \cong E^{*} ; \quad\left(A_{I}\right)_{\alpha}^{\beta}=\nabla_{\beta} s_{\alpha},
\end{aligned}
$$

where

$$
\begin{aligned}
& \nabla s\left(\frac{\partial}{\partial y^{i}}\right)=\left(\nabla_{i} s_{\alpha}\right) \frac{\partial}{\partial q^{\alpha}}, \quad \nabla_{i} s_{\alpha}:=\frac{\partial s_{\alpha}}{\partial y^{j}}+s_{\beta} \frac{\partial R_{j}^{\beta}}{\partial q^{\alpha}}, \\
& \nabla s\left(\frac{\partial}{\partial q^{\beta}}\right)=\left(\nabla_{\beta} s_{\alpha}\right) \frac{\partial}{\partial q^{\alpha}}, \quad \nabla_{\beta} s_{\alpha}:=\frac{\partial s_{\alpha}}{\partial q^{\beta}},
\end{aligned}
$$

Finally we note that

$$
\omega_{U}(s)\left(e_{i}, e_{j}\right)=w_{i j}-s_{\beta} F_{i j}^{\beta}:=\widetilde{\omega}_{i j}
$$

and denote its inverse by $\left(\widetilde{\omega}^{i j}\right)$. Note that $\left(\widetilde{\omega}_{i j}\right)$ is invertible if $s_{\beta}$ is sufficiently small, i.e., if the section $s$ is $C^{0}$-close to the zero section, or its image stays inside of $U$. Now Proposition 2.2 immediately implies

Theorem 7.1. Let $\nabla s$ be the vertical projection of $d s$ as in 7.3). Then the graph of the section $s: Y \rightarrow U$ is coisotropic with respect to $\omega_{U}$ if and only if $s$ satisfies

$$
\nabla_{i} s_{\alpha} \widetilde{\omega}^{i j} \nabla_{j} s_{\beta}=\nabla_{\beta} s_{\alpha}-\nabla_{\alpha} s_{\beta}
$$

for all $\alpha>\beta$ or

$$
\frac{1}{2}\left(\nabla_{i} s_{\alpha} \widetilde{\omega}^{i j} \nabla_{j} s_{\beta}\right) f_{\alpha}^{*} \wedge f_{\beta}^{*}=\left(\nabla_{\beta} s_{\alpha}\right) f_{\alpha}^{*} \wedge f_{\beta}^{*}
$$

where $f_{\alpha}^{*}$ is the dual frame of $\left\{\frac{\partial}{\partial q^{1}}, \cdots, \frac{\partial}{\partial q^{n-k}}\right\}$ defined by 6.5 . We call any of the two the master equation for the pre-symplectic manifold $(Y, \omega)$.

Remark 7.2. It is straightforward to check that both sides of (7.6) are independent of the local lifting $E^{\sharp}$ but depends only on the splitting $\Pi: T Y=G \oplus T \mathcal{F}$. In fact, the right hand side does not depend on this splitting either but depends only on the null foliation $\mathcal{F}$, which is nothing but $-d_{\mathcal{F}}(s)$ where $d_{\mathcal{F}}$ is the exterior derivative of $s \in \Omega^{1}(\mathcal{F})=\Gamma\left(T^{*} \mathcal{F}\right.$ ) along the foliation (See e.g., [Ha] or section 8 later for the definition of $d_{\mathcal{F}}$ ). The latter property follows from Remark 7.1. On the other hand, the invariant meaning of the left hand side is not completely clear. One may interpret it as a kind of twisted bracket which varies along the value of the section itself. 
Note that (7.6) involves terms of all order of $s_{\beta}$ because the matrix $\left(\widetilde{\omega}^{i j}\right)$ is the inverse of the matrix

$$
\widetilde{\omega}_{i j}=\omega_{i j}-s_{\beta} F_{i j}^{\beta} .
$$

There is a special case where the curvature vanishes i.e., satisfies

$$
F_{G}=F_{i j}^{\beta} \frac{\partial}{\partial q^{\beta}} \otimes d y^{i} \wedge d y^{j}=0
$$

in addition to (4.3). In this case, $\widetilde{\omega}_{i j}=\omega_{i j}$ which depends only on $y^{i}$ 's and so does $\omega^{i j}$. Therefore (7.6) is reduced to the quadratic equation

$$
\frac{1}{2}\left(\nabla_{i} s_{\alpha} \omega^{i j} \nabla_{j} s_{\beta}\right) f_{\alpha}^{*} \wedge f_{\beta}^{*}=\left(\nabla_{\beta} s_{\alpha}\right) f_{\alpha}^{*} \wedge f_{\beta}^{*} .
$$

\section{Hamiltonian equivalence}

In this section, we give the proof of Theorem 3.3 and clarify the relation between the intrinsic equivalence between the pre-symplectic structures and the $e x$ trinsic equivalence between coisotropic embeddings in $U$. The intrinsic equivalence is provided by the pre-Hamiltonian diffeomorphisms on the pre-symplectic manifold $(Y, \omega)$ and the extrinsic ones by Hamiltonian deformations of its coisotropic embedding into $\left(U, \omega_{U}\right)$ (and so into any symplectic $\left(X, \omega_{X}\right)$ that allows a coisotropic embedding of $(Y, \omega))$, as far as the Hamiltonian deformations are small enough).

We first recall the theorem stated in section 3 and provide its proof.

Theorem 8.1. Any locally pre-Hamiltonian (respectively, pre-Hamiltonian) vector field $\xi$ on a pre-symplectic manifold $(Y, \omega)$ can be extended to a locally Hamiltonian (respectively, Hamiltonian) vector field on the thickening $\left(U, \omega_{U}\right)$.

Proof. Let $\mathcal{F}$ be the null foliation of $(Y, \omega)$ and $\pi^{-1}(\mathcal{F})$ be the induced foliation on the canonical thickening $\pi: U \rightarrow Y$. Choose any splitting $T Y=G \oplus E$ and write

$$
\xi=\xi_{G}+\xi_{E} .
$$

First consider the case when $\xi$ is locally pre-Hamiltonian, i.e., $d(\xi\rfloor \omega)=0$. The pre-Hamiltonian case will follow immediately from the proof of this. Since $\left.\omega\right|_{E} \equiv 0$, $\left.\xi\rfloor \omega=\xi_{G}\right\rfloor \omega$ and so we have $\left.d\left(\xi_{G}\right\rfloor \omega\right)=0$. We denote by

$$
G^{\sharp}:=\left(T\left(\pi^{-1}(\mathcal{F})\right)\right)^{\omega_{U}}
$$

and decompose $T U=G^{\sharp} \oplus T\left(\pi^{-1}(\mathcal{F})\right)$. We will find a vector field $\Xi$ on $U$ of the form

$$
\Xi=\Xi_{G}+\Xi_{E}
$$

where $\Xi_{G}(\alpha) \in G_{\alpha}^{\sharp}$ and $\Xi_{E}(\alpha) \in T_{\alpha}\left(\pi^{-1}(\mathcal{F})\right)$.

First we determine $\Xi_{E}$. We define a function $f_{\xi}: U \rightarrow \mathbb{R}$ by

$$
f_{\xi}(\alpha)=\left\langle\alpha, \xi_{E}\right\rangle
$$

for $\alpha \in U \subset E^{*}$ and by $X_{f_{\xi}}$ its Hamiltonian vector field on $U$ with respect to the symplectic form $\omega_{U}$. Note that $\left.X_{f_{\xi}}\right|_{Y}=\xi_{E}$. Motivated by this, we just set

$$
\Xi_{E}=X_{f_{\xi}} \text {. }
$$

Determination of $\Xi_{G}$ is now in order. With the above choice of $\Xi_{E}$, we have

$$
\left.\left.d(\Xi\rfloor \omega_{U}\right)=d\left(\Xi_{G}\right\rfloor \omega_{U}\right) .
$$


We would like to solve the equation

$$
\left\{\begin{array}{l}
d\left(\Xi_{G} \mid \omega_{U}\right)=0 \\
\left.\Xi_{G}\right|_{Y}=\xi_{G}
\end{array}\right.
$$

Note the initial condition and the condition $\Xi_{G} \in G^{\sharp}$ imply

$$
\left.\left.\left(\Xi_{G}\right\rfloor \omega_{U}\right)\left.\right|_{Y}=\xi\right\rfloor \omega .
$$

Noting that $\omega_{U}$ is nondegenerate, we will therefore set

$$
\left.\Theta:=\Xi_{G}\right\rfloor \omega_{U}
$$

and solve the following extension problem of one-forms instead.

$$
\left.d \Theta=0,\left.\quad \Theta\right|_{Y}=\xi\right\rfloor \omega .
$$

This can be solved in a neighborhood of $Y \subset E^{*}$ by the standard homotopy method [We1], when the initial one-form $\xi\rfloor \omega$ on $Y \subset U$ is closed which is precisely the condition for $\xi$ to be locally pre-Hamiltonian. Furthermore the homotopy method also leads to an exact extension when the initial form $\xi\rfloor \omega$ is exact, i.e., when $\xi$ is pre-Hamiltonian. This finishes the proof.

Next, we consider the coordinate expression of (8.3). In the canonical coordinates

$$
\left(y^{1}, \cdots, y^{2 k}, q^{1}, \cdots, q^{n-k}, p_{1}, \cdots, p_{n-k}\right),
$$

we write

$$
\Xi_{G}=\Xi_{G}^{j} e_{j}=\Xi_{G}^{j}\left(\frac{\partial}{\partial y^{j}}+R_{j}^{\alpha} \frac{\partial}{\partial q^{\alpha}}-p_{\beta} \frac{\partial R_{j}^{\beta}}{\partial q^{\nu}} \frac{\partial}{\partial p_{\nu}}\right)
$$

where $\Xi_{G}^{j}$ 's are the coordinate functions of $\Xi_{G}$ with respect to the basis $\left\{e_{j}\right\}_{1 \leq j \leq 2 k}$ of $G^{\sharp}$. Then a straightforward calculation using the formula (6.8) shows that (8.3) becomes

$$
\left\{\begin{array}{l}
d\left(\Xi_{G}^{i}\left(\omega_{i j}-p_{\beta} F_{i j}^{\beta}\right) d y^{j}\right)=0 \\
\Xi_{G}^{i}\left(y^{1}, \cdots, y^{2 k}, q^{1}, \cdots, q^{n-k}, 0, \cdots, 0\right)=\xi_{G}^{i}\left(y^{1}, \cdots, y^{2 k}, q^{1}, \cdots, q^{n-k}\right)
\end{array}\right.
$$

where $\xi_{G}^{i}$ is the coordinates of $\xi_{G}$

$$
\xi_{G}=\xi_{G}^{i}\left(\frac{\partial}{\partial y^{i}}+R_{i}^{\alpha} \frac{\partial}{\partial q^{\alpha}}\right) .
$$

In particular we have the following immediate corollary.

Corollary 8.2. Suppose $F_{\Pi} \equiv 0$. Then $\Xi=\xi_{G}^{\sharp}+X_{f_{\xi}}$ is the locally Hamiltonian (respectively Hamiltonian) vector field on $\left(U, \omega_{U}\right)$ which extends the locally preHamiltonian (respectively pre-Hamiltonian) vector field $\xi$ on $(Y, \omega)$.

Proof. When $F_{\Pi} \equiv 0$, (8.5) becomes

$$
d\left(\Xi_{G}^{i} \omega_{i j} d y^{j}\right)=0
$$

Since $\omega_{i j}$ depends only on $y^{i}$ s and $d\left(\xi_{G}^{i} \omega_{i j} d y^{j}\right)=0$, the $p_{\alpha}$-independent function

$$
\Xi_{G}^{i}=\xi_{G}^{i}
$$

provides a solution for (8.5), which precisely corresponds to the extension

$$
\Xi=\xi_{G}^{\sharp}+X_{f_{\xi}} .
$$

This finishes the proof. 


\section{Strong homotopy Lie Algebroid}

In this section, we unravel the algebraic structure that provides an invariant description of the master equation (7.6) in the formal level.

9.1. Lie algebroid and its cohomology. We start with recalling the definition of Lie algebroid and its associated $E$-de Rham complex and $E$-cohomology. The leafwise de Rham complex $\Omega^{\bullet}(\mathcal{F})$ is a special case of the E-de Rham complex associated to the general Lie algebroid $E$, We quote the following definitions from $[\mathrm{NT}]$.

Definition 9.1. Let $M$ be a smooth manifold. A Lie algebroid on $M$ is a triple $(E, \rho,[]$,$) , where E$ is a vector bundle on $M,[$,$] is a Lie algebra structure on the$ sheaf of sections of $E$, and $\rho$ is a bundle map, called the anchor map,

$$
\rho: E \rightarrow T M
$$

such that the induced map

$$
\Gamma(\rho): \Gamma(M ; E) \rightarrow \Gamma(T M)
$$

is a Lie algebra homomorphism and, for any sections $\sigma$ and $\tau$ of $E$ and a smooth function $f$ on $M$, the identity

$$
[\sigma, f \tau]=\rho(\sigma)[f] \cdot \tau+f \cdot[\sigma, \tau] .
$$

Definition 9.2. Let $(E, \rho,[]$,$) be a Lie algebroid on M$. The $E$-de Rham complex $\left({ }^{E} \Omega^{\bullet}(M),{ }^{E} d\right)$ is defined by

$$
\begin{aligned}
{ }^{E} \Omega\left(\Lambda^{\bullet}\left(E^{*}\right)\right)= & \Gamma\left(\Lambda^{\bullet}\left(E^{*}\right)\right) \\
{ }^{E} d \omega\left(\sigma_{1}, \cdots, \sigma_{k+1}\right)= & \sum_{i}(-1)^{i} \rho\left(\sigma_{i}\right) \omega\left(\sigma_{1}, \cdots, \widehat{\sigma}_{i}, \cdots, \sigma_{k+1}\right) \\
& +\sum_{i<j}(-1)^{i+j-1} \omega\left(\left[\sigma_{i}, \sigma_{j}\right], \sigma_{1}, \cdots, \widehat{\sigma}_{i}, \cdots, \widehat{\sigma}_{j}, \cdots, \sigma_{k+1}\right) .
\end{aligned}
$$

The cohomology of this complex will be denoted by ${ }^{E} H^{*}(M)$ and called the $E$-de Rham cohomology of $M$.

In our case, $M=Y$ and $E:=T Y^{\omega}=T \mathcal{F}$ and the anchor map $\rho: E \rightarrow T Y$ is nothing but the inclusion map $i: T Y^{\omega} \rightarrow T Y$. The integrability of $T Y^{\omega}$ implies that the restriction of the Lie bracket on $\Gamma(T Y)$ to $\Gamma\left(T Y^{\omega}\right)$ defines the Lie bracket $[$,$] on \Gamma(E)$. Therefore the triple

$$
\left(E=T Y^{\omega}, \rho=i,[,]\right)
$$

defines the structure of Lie algebroid and hence the $E$-differential. In our case, the corresponding $E$-differential is nothing but $d_{\mathcal{F}}$ the exterior derivative along the null foliation $\mathcal{F}$ and its cohomology, the cohomology $H^{*}(\mathcal{F})$ of the foliation $\mathcal{F}$.

Now we derive, as a first step towards the study of the master equation, the linearized version of the master equation which characterizes the infinitesimal deformation space of coisotropic submanifolds. For this, we introduce the space

$$
\text { Coiso }_{k}=\text { Coiso }_{k}\left(X, \omega_{X}\right)
$$

the set of coisotropic submanifolds with nullity $n-k$ for $0 \leq k \leq n$ and characterize its infinitesimal deformation space at $Y \subset E^{*}$, the zero section of $E^{*}$. As Zambon [Za] observed (see Example 10.4 later), this space does not form a smooth Frechet 
manifold unlike the Lagrangian case. By the coisotropic neighborhood theorem, the infinitesimal deformation space, denoted as $T_{Y} \mathcal{C o i s o}_{k}\left(X, \omega_{X}\right)=T_{Y} \mathcal{C}_{\text {oiso }}\left(U, \omega_{U}\right)$ with some abuse of notion, depends only on $(Y, \omega)$ where $\omega=i^{*} \omega_{X}$, but not on $\left(X, \omega_{X}\right)$. An element in $T_{Y} \mathcal{C}$ iso $_{k}\left(U, \omega_{U}\right)$ is a section of the bundle $E^{*}=T^{*} \mathcal{F} \rightarrow Y$.

The following characterizes the condition for a section $\xi$ to be an infinitesimal deformation of $Y$.

Theorem 9.1. A section $\xi \in \Omega^{1}(\mathcal{F})$ is an infinitesimal deformation of coisotropic submanifold $Y$ if and only if $\xi$ satisfies

$$
d_{\mathcal{F}}(\xi)=0 \text { on } \Omega^{2}(\mathcal{F}),
$$

where $d_{\mathcal{F}}$ is the exterior derivative along the leaves of the foliation $\mathcal{F}$.

Proof. This is an immediate consequence of higher order nature of the left hand side in (7.6) and the definition of $d_{\mathcal{F}}$.

Now we need to mod out the solution space of (9.1) by certain gauge equivalence classes. Algebraically, the set of equivalence classes is the first cohomology of the null foliation $\mathcal{F}$

$$
H^{1}(\mathcal{F}):=\left.\operatorname{ker} d_{\mathcal{F}}\right|_{\Omega^{1}(\mathcal{F})} /\left.\operatorname{im} d_{\mathcal{F}}\right|_{\Omega^{0}(\mathcal{F})}
$$

of the graded algebra $\left(\Omega^{\bullet}(\mathcal{F}), d_{\mathcal{F}}\right)$, which is the leafwise de Rham complex of the foliation $\mathcal{F}$. Geometrically we are considering the set of equivalence classes of pre-symplectic structures up to the pre-Hamiltonian diffeomorphisms on $(P, \omega)$. Or equivalently the latter is (locally) equivalent to the set of deformations of coisotropic embeddings into $\left(U, \omega_{U}\right)$ (or any into $\left(X, \omega_{X}\right)$ into which $(Y, \omega)$ is coisotropically embedded) of $(Y, \omega)$ up to the ambient Hamiltonian isotopies. See section 8

Since the leafwise de Rham cohomology $H^{\bullet}(\mathcal{F})$ is determined by $(Y, \omega)$, we will denote

$$
H^{\bullet}(Y, \omega):=H^{\bullet}(\mathcal{F})
$$

and call the cohomology of (the null foliation of) $(Y, \omega)$. In fact, it depends only on the foliation $\mathcal{F}$. When $k=0$, this reduces to the standard description of the deformation problem of the Lagrangian submanifolds and $H^{1}(Y, \omega)$ becomes the standard de Rham cohomology $H^{1}(Y ; \mathbb{R})$. When $k=n$, it becomes $C^{\infty}(X, \omega)$ which carries the Poisson bracket associated to the symplectic form $\omega$. The cohomology $H^{1}(Y, \omega)$ is a mixture of these two. Except the case of $k=0$, this cohomology is infinite dimensional in general.

9.2. Review of strong homotopy Lie algebra. In this section we give a brief review of the definition of the strong homotopy Lie algebra or the $L_{\infty}$ algebra. We will follow Fukaya's exposition verbatim on the part of $L_{\infty}$ algebra from [Fu]. In particular, we will follow the sign convention used in $[\mathrm{Fu}]$ which is also the same as that of [FOOO] in the context of the $A_{\infty}$-algebra case. We will extract only the essentials that are needed to give a self-contained definition of our strong homotopy Lie algebroid. For more details of the discussion on the $L_{\infty}$ algebra, we refer to [Fu] or [K1] (with different sign convention) also to [AKKS], [P1] for the exposition in the context of the Batalin-Vilkovisky approach using the notion of super-manifolds.

Let $C$ be a graded $R$-module where $R$ is the coefficient ring. In our case, $R$ will be either $\mathbb{R}$ or $\mathbb{C}$. We denote by $C[1]$ its suspension defined by

$$
C[1]^{k}=C^{k+1} .
$$


We denote by $\operatorname{deg}(x)=|x|$ the degree of $x \in C$ before the shift and $\operatorname{deg}^{\prime}(x)=|x|^{\prime}$ that after the degree shifting, i.e., $|x|^{\prime}=|x|-1$. Define the bar complex $B(C[1])$ by

$$
B_{k}(C[1])=(C[1])^{k \otimes}, \quad B(C[1])=\bigoplus_{k=0}^{\infty} B_{k}(C[1]) .
$$

Here $B_{0}(C[1])=R$ by definition. We provide the degree of elements of $B(C[1])$ by the rule

$$
\left|x_{1} \otimes \cdots \otimes x_{k}\right|^{\prime}:=\sum_{i=1}^{k}\left|x_{i}\right|^{\prime}=\sum_{i=1}^{k}\left|x_{i}\right|-k
$$

where $|\cdot|^{\prime}$ is the shifted degree. There is a natural coproduct

$$
\Delta: B(C[1]) \rightarrow B(C[1]) \otimes B(C[1])
$$

on $B(C[1])$ defined by

$$
\Delta\left(x_{1} \otimes \cdots x_{k}\right)=\sum_{i=0}^{k}\left(x_{1} \otimes \cdots \otimes x_{i}\right) \otimes\left(x_{i+1} \otimes \cdots \otimes x_{k}\right) .
$$

Finally we have the natural projection

$$
\varepsilon: B(C[1]) \rightarrow\left(B(C[1])^{0} \cong R .\right.
$$

Then the triple $(B(C[1]), \Delta, \varepsilon)$ defines the structure of graded coalgebra.

Now we consider the action of the symmetric group $S_{k}$ on $B_{k}(C[1])$ by the permutation of arguments in the tensor powers

$$
\sigma\left(x_{1} \otimes \cdots \otimes x_{k}\right)=(-1)^{|\sigma|} x_{\sigma(1)} \otimes \cdots \otimes x_{\sigma(k)}
$$

where $|\sigma|$ is defined to be

$$
|\sigma|=\sum_{i, j ; i<j, \sigma(i)>\sigma(j)}\left|x_{i}\right|^{\prime}\left|x_{j}\right|^{\prime} .
$$

We define $E_{k}(C[1])$ to be the submodule of $B_{k}(C[1])$ consisting of fixed points of the $S_{k}$-action defined above, and

$$
E(C[1])=\bigoplus_{i=0}^{\infty} E_{k}(C[1]) .
$$

The above coproduct (9.3) naturally induces a coproduct on $E(C[1])$, which we also denote by $\Delta$. And the projection $\varepsilon$ induces the projection on $E(C[1])$ again denoted by $\varepsilon$. Then we have the following lemma

Lemma 9.2. [Lemma 8.3.1, Fu] The triple $(E(C[1]), \Delta, \varepsilon)$ is a graded-cocommutative coalgebra.

Definition 9.3. [Definition 8.3.2, Fu] The structure of $L_{\infty}$ algebra or strong homotopy Lie algebra is a sequence of $R$ module homomorphisms

$$
\mathfrak{m}_{k}: E_{k}(C[1]) \rightarrow C[1], \quad k=1,2, \cdots,
$$

of degree +1 such that the coderivation

$$
\delta=\sum_{k=1}^{\infty} \widehat{\mathfrak{m}}_{k}
$$

satisfies $\delta \delta=0$. Here we denote by $\widehat{\mathfrak{m}}_{k}: E(C[1]) \rightarrow E(C[1])$ the unique extension of $\mathfrak{m}_{k}$ as a coderivation on $E(C[1])$. 
One can write the condition $\delta \delta=0$ more explicitly as

$$
\begin{gathered}
\sum_{k=1}^{n} \sum_{i=1}^{n-k+1}(-1)^{\left|x_{1}\right|^{\prime}+\cdots+\left|x_{i-1}\right|^{\prime}} \mathfrak{m}_{n-k+1}\left(x_{1} \otimes \cdots \otimes x_{i-1} \otimes \mathfrak{m}_{k}\left(x_{i} \otimes \cdots \otimes x_{i+k-1}\right)\right. \\
\left.\otimes x_{i+k} \otimes \cdots \otimes x_{n}\right)=0
\end{gathered}
$$

for any $n=1,2, \cdots$. In particular, we have $\mathfrak{m}_{1} \mathfrak{m}_{1}=0$ and so it defines complex $\left(C, \mathfrak{m}_{1}\right)$. We define the $\mathfrak{m}_{1}$-cohomology by

$$
H\left(C, \mathfrak{m}_{1}\right)=\text { ker } \mathfrak{m}_{1} / \mathrm{im} \mathfrak{m}_{1} .
$$

A weak $L_{\infty}$-algebra is defined in the same way, except that it also includes the $\mathfrak{m}_{0}$-term

$$
\mathfrak{m}_{0}: R \rightarrow E(C[1]) .
$$

The first two terms of the $L_{\infty}$ relation for a weak $L_{\infty}$ algebra are given as

$$
\begin{aligned}
\mathfrak{m}_{1}\left(\mathfrak{m}_{0}(1)\right) & =0 \\
\mathfrak{m}_{1} \mathfrak{m}_{1}(x)+(-1)^{|x|^{\prime}} \mathfrak{m}_{2}\left(x, \mathfrak{m}_{0}(1)\right)+\mathfrak{m}_{2}\left(\mathfrak{m}_{0}(1), x\right) & =0 .
\end{aligned}
$$

In particular, for the case of weak $L_{\infty}$ algebras, $\mathfrak{m}_{1}$ will not satisfy boundary property, i.e., $\mathfrak{m}_{1} \mathfrak{m}_{1} \neq 0$ in general. We will explain in the appendix of the present paper that the vanishing of $\mathfrak{m}_{0}$ is closely related to the coisotropic boundary condition via the Batalin-Vilkovisky formulation of the open string $A$-model. See also Lemma 9.5

9.3. Definition of strong homotopy Lie algebroid. Now we are ready to introduce the main definition of our strong homotopy Lie algebroid

Definition 9.4. Let $E \rightarrow Y$ be a Lie algebroid. An $L_{\infty}$-structure over the Lie algebroid is a structure of strong homotopy Lie algebra $(\mathfrak{r}[1], \mathfrak{m})$ on the associated $E$-de Rham complex $\mathfrak{l}^{\bullet}=\Omega^{\bullet}(E)=\Gamma\left(\Lambda^{\bullet}\left(E^{*}\right)\right)$ such that $\mathfrak{m}_{1}$ is the $E$-differential ${ }^{E} d$ induced by the Lie algebroid structure on $E$ as described in section 9.1 We call the pair $(E \rightarrow Y, \mathfrak{m})$ a strong homotopy Lie algebroid.

With this definition of strong homotopy Lie algebroid, we will show that for given presymplectic manifold $(Y, \omega)$ each splitting $\Pi: T Y=G \oplus T \mathcal{F}$ induces a canonical $L_{\infty}$-structure over the Lie algebroid $T \mathcal{F} \rightarrow Y$.

We recall that $U \subset E^{*}=T^{*} \mathcal{F}$ and we have chosen the (locally defined) transverse symplectic connection

$$
T U=\left(G^{\sharp} \oplus E^{\sharp}\right) \oplus V T U
$$

where $E^{\sharp}$ is given as in (7.1). This induces a locally defined leafwise symplectic connection of $T U \rightarrow Y$ which we denote by $\nabla$.

The crucial structures relevant to the invariant description of this structure will be a linear map

$$
\widetilde{\omega}: \Omega^{1}\left(Y ; \Lambda^{\bullet} E^{*}\right) \rightarrow \Gamma\left(\Lambda^{\bullet+1} E^{*}\right)=\Omega^{\bullet+1}(\mathcal{F}),
$$

a quadratic map

$$
\langle\cdot, \cdot\rangle_{\omega}: \Omega^{1}\left(Y ; \Lambda^{\ell_{1}} E^{*}\right) \otimes \Omega^{1}\left(Y ; \Lambda^{\ell_{2}} E^{*}\right) \rightarrow \Omega^{\ell_{1}+\ell_{2}}(\mathcal{F}),
$$

and the third map that is induced by the transverse $\Pi$-curvature.

Now we describe those maps. The linear map $\widetilde{\omega}$ is defined by

$$
\widetilde{\omega}(A):=\left(\left.A\right|_{E}\right)_{\text {skew }}:
$$


Here note that an element $A \in \Omega^{1}\left(Y ; \Lambda^{k} E^{*}\right)$ a section of $T^{*} Y \otimes \Lambda^{k} E^{*} .\left.A\right|_{E}$ is the element in $E^{*} \otimes \Lambda^{k} E^{*}$ obtained by restricting $A$ to $E$ for the first factor, and then $\left(\left.A\right|_{E}\right)_{\text {skew }}$ is the skew-symmetrization of $\left.A\right|_{E}$.

The quadratic map is defined by

$$
\langle A, B\rangle_{\omega}:=\langle A|\pi| B\rangle-\langle B|\pi| A\rangle
$$

where $\pi$ is the transverse Poisson bi-vector on $N^{*} \mathcal{F}$ associated to the transverse symplectic form $\omega$ on $N \mathcal{F}$.

Finally we define two maps involving the transverse $\Pi$-curvature $F_{\Pi}$ : For any two form $\eta \in \Omega^{2}(Y)$, we denote

$$
\operatorname{ker} \eta:=\{v \in T Y \mid \eta(v, \cdot) \equiv 0\},
$$

and the subset $\Omega_{\omega}^{2}(Y) \subset \Omega^{2}(Y)$ by

$$
\Omega_{\omega}^{2}(Y):=\left\{\eta \in \Omega^{2}(Y) \mid \operatorname{ker} \eta \supset \operatorname{ker} \omega\right\} .
$$

The first map we will use is the "contraction" by $F_{\Pi}$

$$
\left.\widetilde{F}: \Omega^{\ell}(\mathcal{F}) \rightarrow \Omega_{\omega}^{2}(Y) \otimes \Omega^{\ell-1}(\mathcal{F}) ; \quad \widetilde{F}(\xi)=F\right\rfloor \xi,
$$

where the contraction is taken between $E$ and $E^{*}$. The second map is "raising indices" of $F$ by $\omega^{-1}=\left(\omega^{i j}\right)$ on $T Y / E \cong G$. We will denote

$$
F^{\#}:=F \omega^{-1}=F_{i}^{\alpha j} d y^{i} \otimes\left(\frac{\partial}{\partial y^{j}}+R_{j}^{\beta} \frac{\partial}{\partial q^{\beta}}\right) \otimes \frac{\partial}{\partial q^{\alpha}} \in \Gamma\left(G^{*} \otimes G \otimes E\right),
$$

where $F_{i}^{\alpha j}=F_{i k}^{\alpha} \omega^{k j}$. Note that we can identify $\Gamma\left(G^{*} \otimes G \otimes E\right)$ with $\Gamma\left(N^{*} \mathcal{F} \otimes\right.$ $N \mathcal{F} \otimes E$ ) via the isomorphism $\pi_{G}: G \rightarrow N \mathcal{F}$.

For given $\xi \in \Omega^{\ell}(\mathcal{F})$, we denote

$$
\begin{gathered}
d_{\mathcal{F}}(\xi):=\left(\left.\nabla \xi\right|_{E}\right)_{\text {skew }} \\
\left\{\xi_{1}, \xi_{2}\right\}_{\Pi}:=\left\langle\nabla \xi_{1}, \nabla \xi_{2}\right\rangle_{\omega}=\sum_{i<j} \omega^{i j}\left(\nabla_{i} \xi_{1}\right) \wedge\left(\nabla_{j} \xi_{2}\right) .
\end{gathered}
$$

Here the first map is nothing but the leafwise differential of the null foliation which is indeed independent of the choice of splitting $\Pi: T Y=G \oplus T \mathcal{F}$ but depends only on the foliation. The second is a bracket in the transverse direction which does not satisfy the Jacobi identity in general because of the presence of non-zero transverse curvature of the null foliation. Dependence on $\Pi$ for the bracket comes from the covariant derivative $\nabla_{i} s:=\Pi^{v} \circ d s\left(e_{i}\right)$. Because of this, the structure

$$
\left(\mathfrak{l}=\bigoplus_{j=0}^{n-k} \mathfrak{l}^{j} ; d_{\mathcal{F}},\{\cdot, \cdot\}_{\Pi}\right), \quad \mathfrak{l}^{j}=\Omega^{j}(\mathcal{F})
$$

fails to define a differential graded Lie algebra in general. However we have

Theorem 9.3. Let $\Pi: T Y=G \oplus T \mathcal{F}$ be a splitting such that $F_{\Pi} \equiv 0$. Then 9.14) defines a differential graded Lie algebra. More precisely, we have the identity

$$
d_{\mathcal{F}}\left\{\xi_{1}, \xi_{2}\right\}_{\Pi}=\left\{d_{\mathcal{F}}\left(\xi_{1}\right), \xi_{2}\right\}_{\Pi}+(-1)^{\left|\xi_{1}\right|}\left\{\xi_{1}, d_{\mathcal{F}}\left(\xi_{2}\right)\right\}_{\Pi} .
$$


Proof. Let $\Pi: T Y=G \oplus T \mathcal{F}$ be such a splitting. By definition of $F_{\Pi}$, vanishing of $F_{\Pi}$ means that the distribution $G$ is integrable. Together with the foliation $\mathcal{F}$, we can construct a coordinate system

$$
\left(y^{1}, \cdots, y^{2 k}, q^{1}, \cdots, q^{n-k}\right)
$$

such that

$$
G=\operatorname{span}\left\{\frac{\partial}{\partial y^{1}}, \cdots, \frac{\partial}{\partial y^{2 k}}\right\}, \quad T \mathcal{F}=\operatorname{span}\left\{\frac{\partial}{\partial q^{1}}, \cdots, \frac{\partial}{\partial q^{n-k}}\right\} .
$$

In particular, we have $R_{i}^{\alpha} \equiv 0$ and so $\left[\nabla_{i}, \nabla_{j}\right]=\left[\nabla_{k}, \nabla_{\alpha}\right]=\left[\nabla_{\alpha}, \nabla_{\beta}\right]=0$. Once we have these, (9.15) immediately follows by computing

$$
d_{\mathcal{F}}\left\{\xi_{1}, \xi_{2}\right\}_{\Pi}=d q^{\alpha} \wedge \nabla_{\alpha}\left(\frac{1}{2} \omega^{i j} \nabla_{i} \xi_{1} \wedge \nabla_{j} \xi_{2}\right) .
$$

using the fact that $\omega^{i j}$ is independent of $q^{\alpha}$ 's. This finishes the proof.

In the general case, it turns out that for each given splitting $\Pi: T Y=G \oplus T \mathcal{F}$ the pair $\left(d_{\mathcal{F}},\{\cdot, \cdot\}_{\Pi}\right)$ can be extended to an infinite family of graded multilinear maps

$$
\mathfrak{m}_{\ell}=\mathfrak{m}_{\ell}^{\Pi}:\left(\Omega[1]^{\bullet}(\mathcal{F})\right)^{\otimes \ell} \rightarrow \Omega[1]^{\bullet}(\mathcal{F})
$$

so that the structure

$$
\left(\bigoplus_{j=0}^{n-k} \mathfrak{r}[1]^{j} ;\left\{\mathfrak{m}_{\ell}\right\}_{1 \leq \ell<\infty}\right)
$$

defines a strong homotopy Lie algebroid on $E=T \mathcal{F} \rightarrow Y$ in the above sense. Here $\Omega[1]^{\bullet}(\mathcal{F})$ is the shifted complex of $\Omega^{\bullet}(\mathcal{F})$, i.e., $\Omega[1]^{k}(\mathcal{F})=\Omega^{k+1}(\mathcal{F})$ and $\mathfrak{m}_{1}$ is defined by

and $\mathfrak{m}_{2}$ is given by

$$
\mathfrak{m}_{1}(\xi)=(-1)^{|\xi|} d_{\mathcal{F}}(\xi)
$$

$$
\mathfrak{m}_{2}\left(\xi_{1}, \xi_{2}\right)=(-1)^{\left|\xi_{1}\right|\left(\left|\xi_{2}\right|+1\right)}\left\{\xi_{1}, \xi_{2}\right\}_{\Pi} .
$$

On the un-shifted group $\mathfrak{l}, d_{\mathcal{F}}$ defines a differential of degree 1 and $\{\cdot, \cdot\}_{\omega}$ is a graded bracket of degree 0 and $\mathfrak{m}_{\ell}$ is a map of degree $2-\ell$.

We now define $\mathfrak{m}_{\ell}$ for $\ell \geq 3$. Here enters the transverse $\Pi$-curvature $F=F_{\Pi}$ of the splitting $\Pi$ of the null foliation $\mathcal{F}$. We define

$$
\left.\left.\mathfrak{m}_{\ell}\left(\xi_{1}, \cdots, \xi_{\ell}\right):=\sum_{\sigma \in S_{\ell}}(-1)^{|\sigma|}\left\langle\nabla \xi_{\sigma(1)},\left(F^{\#}\right\rfloor \xi_{\sigma(2)}\right) \cdots\left(F^{\#}\right\rfloor \xi_{\sigma(\ell-1)}\right) \nabla \xi_{\sigma(\ell)}\right\rangle_{\omega}
$$

where $|\sigma|$ is given by the rule written in (9.4). We have obtained our definition of strong homotopy Lie algebroid associated to the coisotropic submanifolds first via the language of super-manifolds and the Batalin-Vilkovisky formalism in the context of coisotropic branes formulated in [AKSZ] and [P1]. The current definition below is a literal translation of the one formulated in the language of formal super manifolds into the language of tensor calculus. For the reader's convenience, we include this original derivation in the context of Batalin-Vilkovisky formalism in the appendix. In fact, the current proof itself is not very different from this in which we just add more mathematical explanations to it. The proof is essentially a consequence of the fact that the symplectic form $\omega_{U}$ is closed. A purely classical proof using the tensor calculus should be also possible, but with paying the price of obscuring the origin, $d \omega_{U}=0$, of the $L_{\infty}$-structure. Because of this, we do not 
pursue carrying out the tensor calculations preferring the proof using the supermanifold and the relevant super-calculus.

Theorem 9.4. Let $(Y, \omega)$ be a pre-symplectic manifold and $\Pi: T Y=G \oplus T \mathcal{F}$ be a splitting. Then $\Pi$ canonically induces a structure of strong homotopy Lie algebroid on $T \mathcal{F}$ in that the graded complex

$$
\left(\bigoplus_{\bullet} \Omega[1]^{\bullet}(\mathcal{F}),\left\{\mathfrak{m}_{\ell}\right\}_{1 \leq \ell<\infty}\right)
$$

defines the structure of strong homotopy Lie algebra. We denote by $\mathfrak{r}[1]_{(Y, \omega ; \Pi)}^{\infty}$ the corresponding strong homotopy Lie algebra.

Proof. We will prove that the coderivation $\delta=\sum_{\ell=1}^{\infty} \widehat{\mathfrak{m}}_{\ell}$ satisfies $\delta \delta=0$. By restricting to the sections $\xi_{i}$ 's supported in a coordinate chart, we will work with coordinate calculations. We first recall from (6.8) the expression of the symplectic form $\omega_{U}$ on $U \subset E^{*}$

$$
\begin{aligned}
\omega= & \frac{1}{2}\left(\omega_{i j}-p_{\beta} F_{i j}^{\beta}\right) d y^{i} \wedge d y^{j} \\
& -\left(d p_{\beta}+p_{\beta} \frac{\partial R_{i}^{\beta}}{\partial q^{\gamma}} d y^{i}\right) \wedge\left(d q^{\delta}-R_{j}^{\delta} d y^{j}\right)
\end{aligned}
$$

In the same coordinates, the corresponding Poisson bi-vector field $P$ has the form

$$
P=\frac{1}{2} \widetilde{\omega}^{i j} e_{i} \wedge e_{j}+\frac{\partial}{\partial q^{\alpha}} \wedge \frac{\partial}{\partial p_{\alpha}}
$$

where the vector field $e_{j}$ are the ones defined by (7.1) (see Appendix for the derivation of this formula). The fact that $d \omega_{U}=0$ is equivalent to the vanishing of Schouten bracket

$$
[P, P]=0
$$

The Poisson tensor defines a map

$$
\delta_{P}(u)=[P, u]
$$

for each multi-vector field $u$. Furthermore, since $[P, P]=0$, it satisfies $\delta_{P} \delta_{P}=$ $\frac{1}{2}[[P, P], \cdot]=0$. A good way of describing this map $\delta_{P}$ is to use the super-language (see Appendix or $[\mathrm{Gz}]$ for an elegant description of this translation). We change the parity of $T U$ along the fiber and denote by $T[1] U$ the corresponding super tangent bundle of $U$. One considers a multi-vector field on $U$ as a (fiberwise) polynomial function on $T^{*}[1] U$. For example, the Poisson tensor $P$ defines a quadratic function, which we denote by $H$. This also coincides with the push-forward of the canonical even function $H^{*}: T[1] U \rightarrow \mathbb{R}$ induced by the symplectic form $\omega_{U}$. On the other hand, the exterior differential $d$ defines an odd vector field on $T[1] U$, which we denote by $Q$. This vector field is nothing but the pull-back of the Hamiltonian vector field of $H$ with respect to the canonical (odd) symplectic form $\Omega$ on $T^{*}[1] X$. We warn readers that $\Omega$ should not be confused with the symplectic form $\omega_{U}$ itself on $U$. We denote by $\{\cdot, \cdot\}_{\Omega}$ the (super-)Poisson bracket associated to the odd symplectic form $\Omega$ on $T[1] X$. Then we have the identity

$$
Q=\left\{H^{*}, \cdot\right\}_{\Omega}
$$

as a derivation on the set $\mathcal{O}_{T[1] X}$ of "functions" on $T[1] X$ : Here $\mathcal{O}_{T[1] X}$ is the set of differential forms on $X$ considered as fiberwise polynomial functions on $T[1] X$. 
We refer to Appendix or [Gz] for the precise mathematical meaning for this correspondence.

Next we will be interested in whether one can canonically restrict the vector field $Q$ to $\mathbb{L}=T \mathcal{F}[1]$ or equivalently whether the function $H$ has constant value on $\mathbb{L}$. Here comes the coisotropic condition naturally.

Lemma 9.5. Let $H$ be the even function on $T[1] X$ induced by the symplectic form $\omega_{X}$, and $H^{*}: T^{*}[1] X \rightarrow \mathbb{R}$ be its push-forward by the isomorphism $\widetilde{\omega}_{X}: T[1] X \rightarrow$ $T^{*}[1] X$. When $Y \subset(X, \omega)$ is a coisotropic submanifold we have $\left.H^{*}\right|_{N^{*}[1] Y}=0$. Conversely, any (conic) Lagrangian subspace $\mathbb{L}^{*} \subset T^{*}[1] X$ satisfying $\left.H^{*}\right|_{\mathbb{L}^{*}}=0$ is equivalent to $N^{*}[1] Y$, for some coisotropic submanifold $Y$ of $(X, \omega)$.

Proof. This is essentially a translation of the definitions of coisotropic submanifolds and the even function $H^{*}$. We first note that $T_{x} Y$ being coisotropic in $\left(T_{x} X, \omega_{X}(x)\right)$ is equivalent to $N_{x}^{*} Y$ being isotropic in $\left(T_{x}^{*} X, \pi_{X}(x)\right)$ where $\pi_{X}$ is the Poisson tensor associated to $\omega_{X}$. Then it follows from an easy super algebra that this last statement is equivalent to the vanishing of the associated even function $H^{*}$ on $N^{*}[1] Y$ at each point $x \in Y$. This finishes the first part of the theorem.

For the proof of the converse, we first recall that any conic Lagrangian submanifold in $T^{*} X$ has the form of $N^{*} Y$ for some submanifold $Y \subset X$. Then the above argument shows that vanishing of $H^{*}$ on $\mathbb{L}^{*}=N^{*}[1] Y$ is equivalent to $Y$ being coisotropic. This finishes the proof.

Remark 9.5. Note that this lemma, as it is, applies to the coisotropic submanifolds in Poisson manifolds. See [We2] for the definition of coisotropic submanifolds in Poisson manifolds. In our case of $U \subset E^{*}, \pi_{X}$ is nothing but $P$ above.

Noting that $\mathbb{L}^{*}=N^{*}[1] Y$ is mapped to $\mathbb{L}=T \mathcal{F}[1]$ under the isomorphism $\widetilde{\omega}_{X}$, this lemma enables us to restrict the odd vector field $Q$ to $T \mathcal{F}[1]$. We need to describe the Lagrangian embedding $T \mathcal{F}[1] \subset T[1] X$ more explicitly, and describe the induced directional derivative acting on

$$
\Omega^{\bullet}(\mathcal{F})
$$

regarded as a subset of "functions" $T \mathcal{F}[1]$. (Again we refer to Appendix or [Gz] for the precise explanations of this). A more tensorial way of saying this is as follows: Noting that the Poisson tensor pairs with any section of $N^{*} \mathcal{F}$ to give zero due to the coisotropic condition of $Y \subset U$, the Hamiltonian operator [GD]

$$
\delta_{P}:=[P, \cdot]
$$

restricts to $\Omega^{\bullet}(\mathcal{F})=\Gamma\left(\wedge^{\bullet}\left(T^{*} \mathcal{F}\right)\right)$. We denote by $\delta^{\prime}$ this restriction. More precisely $\delta^{\prime}: \Omega^{\bullet}(\mathcal{F}) \rightarrow \Omega^{\bullet}(\mathcal{F})$ is given by the formula

$$
\delta^{\prime}(\xi)=\left.\{H, \widetilde{\xi}\}_{\Omega}\right|_{\mathbb{L}}
$$

where $\widetilde{\xi}$ is the extension of $\xi$ in a neighborhood of $\mathbb{L} \subset T[1] X$ : the extension that we use is the lifting of $\xi \in \Omega^{\bullet}(\mathcal{F})$ to an element of $\Omega^{\bullet}(U)$ obtained by the (local) Ehresman connection constructed in section 7 The condition $\left.Q\right|_{\mathbb{L}} \equiv 0$ implies that this formula is independent of the choice of (local) Ehresman connection. We will just denote $\delta^{\prime}(\xi)=\{H, \xi\}_{\Omega}$ instead of (9.21) as long as there is no danger of confusion.

Obviously, $\delta^{\prime}$ satisfies $\delta^{\prime} \delta^{\prime}=0$ because of $\delta_{P}$ does. Now it remains to verify that this is translated into the $L_{\infty}$ relation $\delta \delta=0$ in the tensorial language which is 
exactly what we wanted to prove. For this purpose, we need to describe the map $\delta^{\prime}: \Omega^{\bullet}(\mathcal{F}) \rightarrow \Omega^{\bullet}(\mathcal{F})$ more explicitly.

Restricting ourselves to a Darboux neighborhood $\mathbb{L}=T \mathcal{F}[1] \subset T[1] U$, we identify the neighborhood with a neighborhood of the zero section $T^{*}[1] \mathbb{L}$. Using the fact that (9.21) depends only on $\xi$, not on the extension, we will make a convenient choice of coordinates to write $H$ in the Darboux neighborhood and describe how the derivation $Q=\{H, \cdot\}_{\Omega}$ acts on $\Omega^{*}(\mathcal{F})$ in the canonical coordinates of $T^{*}[1] \mathbb{L}$. In this way, we can apply the canonical quantization which provides a canonical correspondence between functions on "the phase space" $T^{*}[1] \mathbb{L}$ and the corresponding operators acting on the functions on the "configuration space" $\mathbb{L}$, when we find out how $\delta^{\prime}$ acts on $\Omega^{\bullet}(\mathcal{F})$.

We denote by $\left(y^{i}, q^{\alpha}, p_{\alpha}, y_{i}^{*}, q_{\alpha}^{*}, p_{*}^{\alpha}\right)$ the canonical coordinates $T^{*} \mathbb{L}$ associated with the coordinates $\left(y^{i}, q^{\alpha}, p_{\alpha}\right)$ of $N^{*} \mathcal{F}$. Note that these coordinates are nothing but the canonical coordinates of $N^{*} Y \subset T^{*} U$ pulled-back to $T \mathcal{F} \subset T U$ and its Darboux neighborhood, with the corresponding parity change: We denote the (super) canonical coordinates of $T^{*}[1] \mathbb{L}$ associated with $\left(y^{i}, q^{\alpha} \mid p_{\alpha}\right)$ by

$$
\left(\begin{array}{lll}
y^{i}, & q^{\alpha} & \mid p_{*}^{\alpha} \\
y_{i}^{*}, & q_{\alpha}^{*} & \mid p_{\alpha}
\end{array}\right)
$$

Here we note that the degree of $y^{i}, q^{\alpha}$ and $p_{\alpha}$ are 0 while their anti-fields, i.e., those with $*$ in them have degree 1 . And we want to emphasize that $\mathbb{L}$ is given by the equation

$$
y_{i}^{*}=p_{\alpha}=p_{*}^{\alpha}=0
$$

and $\left(y^{i}, y_{i}^{*}\right),\left(p_{\alpha}, q_{\alpha}^{*}\right)$ and $\left(p_{*}^{\alpha}, q^{\alpha}\right)$ are conjugate variables.

The Poisson tensor $P$ (9.19) becomes the even function $H$ that has the form

$$
H=\frac{1}{2} \widetilde{\omega}^{i j} y_{i}^{\#} y_{j}^{\#}+p_{*}^{\delta} q_{\delta}^{*}
$$

in the canonical coordinates of $T^{*}[1] \mathbb{L}$. Here we define $y_{i}^{\#}$ to be

$$
y_{i}^{\#}:=y_{i}^{*}+R_{i}^{\delta} p_{*}^{\delta}-p_{\beta} \frac{\partial R_{i}^{\beta}}{\partial q^{\delta}} q_{\delta}^{*} .
$$

On the other hand, we have

$$
\left.\widetilde{\omega}_{\alpha}^{-1}=\omega_{\pi(\alpha)}^{-1} \sum_{\ell=0}^{\infty}\left(F^{\#}\right\rfloor \alpha\right)^{\ell} \quad \text { on } T Y / E
$$

(See (11.2) in section 111 later.) which is written as

$$
\widetilde{\omega}_{\alpha}^{i j}=\omega_{\pi(\alpha)}^{i j_{0}} \sum_{\ell=0}^{\infty}\left(p_{\beta_{1}} F_{j_{0}}^{\beta_{1} j_{1}}\right)\left(p_{\beta_{2}} F_{j_{1}}^{\beta_{2} j_{2}}\right) \cdots\left(p_{\beta_{\ell}} F_{j_{\ell-1}}^{\beta_{\ell j}}\right)
$$

in coordinates where $\alpha=p_{\beta} f_{\beta}^{*}$. We make replacements

$$
y_{i}^{*} \mapsto \frac{\partial}{\partial y^{i}}, p_{*}^{\alpha} \mapsto \frac{\partial}{\partial q_{\alpha}^{*}}, p_{\alpha}^{*} \mapsto \frac{\partial}{\partial q^{\alpha}}
$$

following the canonical quantization process in the cotangent bundle, and noting that the derivative $\frac{\partial}{\partial q_{\alpha}^{*}}$ means the contraction by $\frac{\partial}{\partial q^{\alpha}}$ on $\Omega^{\bullet}(\mathcal{F})$. 
Then by expanding the Poisson tensor $P$ or the even function $H$ above into the power series

$$
H=\sum_{\ell=1} H_{\ell}, \quad H_{\ell} \in \mathfrak{r}^{\ell},
$$

in terms of the degree (i.e., the number of factors of odd variables $\left(y_{i}^{*}, p_{*}^{\alpha}, p_{\alpha}\right)$ or the 'ghost number' in the physics language) our definition of $\mathfrak{m}$ exactly corresponds to the $\ell$-linear operator

$$
\left.\left(\xi_{1}, \xi_{2}, \cdots, \xi_{\ell}\right) \mapsto\left\{\cdots\left\{H_{\ell}, \xi_{1}\right\}_{\Omega}, \cdots\right\}_{\Omega}, \xi_{\ell}\right\}_{\Omega} .
$$

Note that the above power series acting on $\left(\xi_{1}, \cdots, \xi_{\ell}\right)$ always reduces to a finite sum and so is well-defined as an operator. Then by definition, the coderivation

$$
\delta=\sum_{\ell=1}^{\infty} \widehat{\mathfrak{m}}_{\ell}
$$

precisely corresponds to $\delta^{\prime}=\{H, \cdot\}_{\Omega}$. The $L_{\infty}$ relation $\delta \delta=0$ then immediately follows from $\delta^{\prime} \delta^{\prime}=0$. This finishes the proof.

For example, under the above translation, the odd vector field

$$
m_{1}=\left.\mathcal{Q}\right|_{\mathbb{L}}
$$

acts on

$$
\mathfrak{l}=\bigoplus_{\ell=0}^{n-k} \mathfrak{l}^{\ell} \cong \bigoplus_{\ell=0}^{n-k} \Omega^{\ell}(\mathcal{F})
$$

is translated into to the leafwise differential $d_{\mathcal{F}}$.

\section{Gauge Equivalence And FORMality Question}

In this section, based on the Lemma 4.1 we prove that two strong homotopy Lie algebroids we have associated to two different splittings are gauge equivalent or $L_{\infty}$-isomorphic. In fact, there exists a canonical $L_{\infty}$ isomorphism between the two which depends only on $B_{\Pi_{0} \Pi}$ given in Lemma 4.1

We first recall the definition of $L_{\infty}$ homomorphism from [Definition 8.3.6, Fu].

Definition 10.1. Let $(C[1], \mathfrak{m}),\left(C^{\prime}[1], \mathfrak{m}^{\prime}\right)$ be $L_{\infty}$ algebras and $\delta, \delta^{\prime}$ be the associated coderivation. A sequence $\varphi=\left\{\varphi_{k}\right\}_{k=1}^{\infty}$ with $\varphi_{k}: E_{k} C[1] \rightarrow C^{\prime}[1]$ is said to be an $L_{\infty}$ homomorphism if the corresponding coalgebra homomorphism $\widehat{\varphi}: E C[1] \rightarrow E C^{\prime}[1]$ satisfies

$$
\widehat{\varphi} \circ \delta=\delta^{\prime} \circ \widehat{\varphi} \text {. }
$$

We say that $\varphi$ is an $L_{\infty}$ isomorphism, if there exists a sequence of homomorphisms $\psi=\left\{\psi_{k}\right\}_{k=1}^{\infty}, \psi: E_{k} C^{\prime}[1] \rightarrow C^{\prime}[1]$ such that its associated coalgebra homomorphism $\widehat{\psi}: E C^{\prime}[1] \rightarrow E C[1]$ satisfies

$$
\widehat{\psi} \circ \widehat{\varphi}=i d_{E C[1]}, \quad \widehat{\varphi} \circ \widehat{\psi}=i d_{E C^{\prime}[1]} .
$$

In this case, we say that two $L_{\infty}$ algebras, $(C[1], \mathfrak{m})$ and $\left(C^{\prime}[1], \mathfrak{m}^{\prime}\right)$ are $L_{\infty}$ isomorphic.

We refer to [Section 8.3, Fu] for more background materials on the $L_{\infty}$ algebra and its homotopy theory, and also to [K1] in the super language on a formal manifold. 
Theorem 10.1. The two structures of strong homotopy Lie algebroid on $T \mathcal{F} \rightarrow Y$ induced by two choices of splitting $\Pi, \Pi^{\prime}$ are canonically $L_{\infty}$ isomorphic.

Proof. We start with the expression of the symplectic form $\omega_{U}$

$$
\omega_{U}=\pi^{*} \omega-d \theta_{G}
$$

given in (3.3) that is canonically constructed on a neighborhood $U$ of the zero section $E^{*}=T^{*} \mathcal{F}$ when a splitting $\Pi: T Y=G \oplus T \mathcal{F}$ is provided. To highlight dependence on the splitting, we denote by $\theta_{\Pi}$ and $\omega_{\Pi}$ the one form $\theta_{G}$ and the symplectic form $\omega_{U}$. We will also denote by $\delta_{\Pi}$ the $\delta: E C[1] \rightarrow E C[1]$ corresponding to the splitting $\Pi$.

Then for a given splitting $\Pi_{0}$, we have

$$
\omega_{\Pi}-\omega_{\Pi_{0}}=d\left(\theta_{\Pi_{0}}-\theta_{\Pi}\right) .
$$

In the super language, this is translated into

$$
H_{\Pi}-H_{\Pi_{0}}=\left\{H_{\Pi_{0}}, \Gamma\right\}_{\Omega}=-\left\{\Gamma, H_{\Pi_{0}}\right\}_{\Omega}
$$

where $\Gamma$ is the function associated to the one-form $\theta_{\Pi_{0}}-\theta_{\Pi}$ which has $\operatorname{deg}^{\prime}(\Gamma)=$ 0 (or equivalently has $\operatorname{deg}(\Gamma)=1$ ). The last identity comes from the supercommutativity of the bracket and the fact that $\operatorname{deg}\left(H_{\Pi_{0}}\right)=2$ and $\operatorname{deg}(\Gamma)=1$. For the simplicity of notations and also to make a connection with the more common notation for the Gerstenhaber bracket as in Appendix, we will simply write

$$
\{A, B\}_{\Omega}=[A, B]
$$

below.

Since any odd element commutes with itself under the bracket $[\cdot, \cdot]$, we have $[\Gamma, \Gamma]=0$ and so we have

$$
\left[\left[H_{\Pi_{0}}, \Gamma\right], \Gamma\right]=0
$$

by the Jacobi identity. This then in turn implies

$$
H_{\Pi_{0}}+\left[H_{\Pi_{0}}, \Gamma\right]=e^{a d_{(-\Gamma)}}\left(H_{0}\right)
$$

where $e^{a d_{(-\Gamma)}}$ is defined by

$$
\begin{aligned}
e^{a d_{(-\Gamma)}(A)} & :=\sum_{k=0} \frac{1}{k !}\left(a d_{(-\Gamma)}\right)^{k} A \\
& =A+[A, \Gamma]+\frac{1}{2 !}[[A, \Gamma], \Gamma]+\cdots .
\end{aligned}
$$

Combining (10.2) and (10.3), we have obtained

$$
H_{\Pi}=e^{a d_{(-\Gamma)}}\left(H_{\Pi_{0}}\right) .
$$

We recall the identity

$$
\left[e^{a d_{(-\Gamma)}} A, e^{a d_{(-\Gamma)}} B\right]=e^{a d_{(-\Gamma)}}[A, B]
$$

which can be rewritten as

$$
a d_{H_{\Pi}}\left(e^{a d_{(-\Gamma)}} A\right)=e^{a d_{(-\Gamma)}}\left(a d_{H_{\Pi_{0}}} A\right)
$$

when it is applied to $B=H_{\Pi_{0}}$. Now we recall from Corollary 5.1 that we have

$$
\left.\left(\theta_{\Pi_{0}}-\theta_{\Pi}\right)\right|_{\left.T E^{*}\right|_{Y}} \equiv 0 \text {. }
$$

Noting that $\mathbb{L}=\left.T \mathcal{F}[1] \subset T E^{*}[1]\right|_{Y}$, this is translated into

$$
\left.\Gamma\right|_{\mathbb{L}} \equiv 0
$$


and so $a d_{\Gamma}$ naturally restricts to the functions on $\mathbb{L}$. We denote by $\widetilde{\varphi}: E C[1] \rightarrow$ $E C[1]$ the coderivation associated to the restriction of $e^{a d_{(-\Gamma)}}$, then (10.4) is precisely translated into the identity

$$
\widetilde{\varphi} \circ \delta_{\Pi_{0}}=\delta_{\Pi} \circ \widetilde{\varphi} .
$$

This proves that $\widetilde{\varphi}$ is an $L_{\infty}$ homomorphism. On the other hand, if $\Gamma^{\prime}$ is the function associated to the one-form $\theta_{\Pi}-\theta_{\Pi_{0}}$, then we have $\Gamma^{\prime}=-\Gamma$ and

$$
H_{\Pi_{0}}=H_{\Pi}+\left[H_{\Pi}, \Gamma^{\prime}\right]=e^{a d_{-\Gamma^{\prime}}\left(H_{\Pi}\right)} .
$$

Now we derive

$$
\begin{aligned}
e^{a d_{\Gamma^{\prime}}} \circ e^{a d_{\Gamma}}(A) & =e^{a d_{\Gamma}}(A)+\left[e^{a d_{\Gamma}}(A), \Gamma^{\prime}\right] \\
& =(A+[A, \Gamma])+\left[A+[A, \Gamma], \Gamma^{\prime}\right]=A
\end{aligned}
$$

for all $A$. Here for the last identity, we use the fact $\Gamma^{\prime}=-\Gamma$ and the identity $\left[[A, \Gamma], \Gamma^{\prime}\right]=0$. The latter follows by the (super)-Jacobi identity using the fact that both $\Gamma$ and $\Gamma^{\prime}$ are odd, and so $\left[\Gamma, \Gamma^{\prime}\right]=0$. If we denote by $\widetilde{\varphi}^{\prime}$ the $L_{\infty}$ homomorphism associated to $\Gamma^{\prime}$, then (10.5) restricted to $\Omega^{\bullet}(\mathcal{F})$ as in (9.21) is translated into the statement that $\widetilde{\varphi}^{\prime}$ is the inverse of $\widetilde{\varphi}$. This finishes the proof.

This theorem then associates a canonical $\left(L_{\infty^{-}}\right)$isomorphism class of strong homotopy Lie algebras to each pre-symplectic manifold and so to each coisotropic submanifold. It is obvious from the construction that pre-Hamiltonian diffeomorphisms induce canonical isomorphism by pull-backs in our strong homotopy Lie algebroids. Pre-symplectic, in particular locally pre-Hamiltonian diffeomorphisms also induce $L_{\infty}$ morphisms which however may not be isomorphisms in general. For example, they do not induce isomorphisms in $H^{\bullet}(\mathcal{F})$ in general, while global pre-Hamiltonian diffeomorphisms do.

In the point of view of coisotropic embeddings this theorem implies that our strong homotopy Lie algebroids for two Hamiltonian isotopic coisotropic submanifolds are canonically isomorphic and so the isomorphism class of the strong homotopy Lie algebroids is an invariant of coisotropic submanifolds modulo the Hamiltonian isotopy. We refer to the next section for the precise explanation on the latter statement.

This enables us to study the moduli problem of deformations of pre-symplectic structures on $Y$ in the similar way as done in [K1], [FOOO], [Fu]. The followings are several interesting questions to ask in this regard, which are analogs to Kontsevich's formality theorem $[\mathrm{K} 1]$ in our case.

Question 10.2. (1) Is the deformation problem formal in the sense of Kontsevich $[\mathrm{K} 1]$ ?

(2) Does the $L_{\infty}$ structure on $\Omega[1]^{\bullet}(Y, \omega)$ always canonically induce an $L_{\infty}$ structure on its $\mathfrak{m}_{1}$-cohomology $H[1]^{\bullet}(Y, \omega)$

$$
\left(\bigoplus H[1] \bullet(Y, \omega),\left\{\overline{\mathfrak{m}}_{\ell}\right\}_{1 \leq \ell<\infty}\right)
$$

with $\overline{\mathfrak{m}}_{1} \equiv 0$. If not, what would be the condition for this to be the case?

(3) If the answer is affirmative in (2), are the two $L_{\infty}$ structures on $\Omega[1]^{\bullet}(\mathcal{F})$ and its cohomology $H[1]^{\bullet}(\mathcal{F})$ quasi-isomorphic? 
When $H^{\bullet}(\mathcal{F})$ is finite dimensional, the proof of [Theorem 8.3.5, Fu] can be imitated and so the answer is affirmative for the questions (2) and (3) in that case. However in general $H^{\bullet}(\mathcal{F})$ will be infinite dimensional. It would be very interesting to see if the proof of [Theorem 8.3.5, Fu] can be generalized to the case where $H^{\bullet}(\mathcal{F})$ is infinite dimensional as in our case.

\section{Moduli Problem and the Kuranishi maP}

In this section, we write down the defining equation (7.6) for the graph Graph $s \subset$ $T U \subset T E^{*}$ to be coisotropic in a formal neighborhood, i.e., in terms of the power series of the section $s$ with respect to the fiber coordinates in $U$.

In this section, we will study the moduli problem of the Maurer-Cartan equation (11.1) in the level of formal power series. With respect to this strong homotopy Lie algebroid constructed in section 9, the formal power series version of (7.6) becomes nothing but the Maurer-Cartan equation of $\mathfrak{r}_{(Y, \omega)}^{\infty}$. We refer to [Gz], [GM], especially to [section 8.3, Fu] for more functorial formulation of the formal moduli problem.

Theorem 11.1. The equation of the formal power series solutions $\Gamma \in \mathfrak{l}^{1}$ of (7.6) is given by

$$
\sum_{\ell=1}^{\infty} \frac{1}{\ell !} \mathfrak{m}_{\ell}(\Gamma, \cdots, \Gamma)=0 \quad \text { on } \Omega^{2}(\mathcal{F})
$$

where

$$
\Gamma=\sum_{k=1}^{\infty} \varepsilon^{k} \Gamma_{k}
$$

where $\Gamma_{k}$ 's are sections of $T^{*} \mathcal{F}$ and $\varepsilon$ is a formal parameter.

Proof. This immediately follows by substituting

$$
s=\Gamma=\sum_{k=0}^{\infty} \varepsilon^{k} \Gamma_{k}
$$

into (7.6) and expanding the matrix $\left(\widetilde{\omega}^{i j}\right)=\left(\omega_{i j}-s_{\beta} F_{i j}^{\beta}\right)^{-1}$ and comparing the result with the definition of $\mathfrak{m}_{\ell}$ 's. Here we invoke the following matrix identity

$$
(A-B)^{-1}=A^{-1}\left(I d-B A^{-1}\right)^{-1}
$$

for $A$ and $(A-B)$ invertible, and so we have

$$
\left.\left.(\omega-F\rfloor s)^{-1}=\omega^{-1}\left(I d-F^{\#}\right\rfloor s\right)^{-1}=\omega^{-1} \sum_{\ell=0}^{\infty}\left(F^{\#}\right\rfloor s\right)^{\ell}
$$

where we recall $F^{\sharp}:=F \omega^{-1}$ from (9.11). Then the proof immediately follows from comparing (11.1) and the definition of $\mathfrak{m}_{k}$ and $\delta=\sum \widehat{\mathfrak{m}}_{\ell}$ (9.17) above.

Remark 11.1. (11.1) has the following interpretation in terms of the deformation problem of presymplectic structures on $(Y, \omega)$ : Following the notation from $[\mathrm{Fu}]$, [FOOO], we denote

$$
e^{\Gamma}:=\sum_{k=0} \frac{1}{k !} \Gamma \otimes \cdots \otimes \Gamma
$$

and write

$$
\mathfrak{m}\left(e^{\Gamma}\right):=\sum \frac{1}{\ell !} \mathfrak{m}_{\ell}(\Gamma, \cdots, \Gamma) .
$$


We define a new family of maps

$$
\mathfrak{m}_{k}^{\Gamma}\left(\xi_{1}, \cdots, \xi_{k}\right)=\mathfrak{m}\left(e^{\Gamma}, \xi_{1}, \cdots, \xi_{k}\right)=\sum_{\ell=1}^{\infty} \frac{1}{\ell !} \mathfrak{m}_{\ell+k}\left(\Gamma, \cdots, \Gamma, \xi_{1}, \cdots, \xi_{k}\right)
$$

for $k \geq 1$, and

$$
\mathfrak{m}_{0}^{\Gamma}(1):=\mathfrak{m}\left(e^{\Gamma}\right)=\sum_{\ell=1}^{\infty} \frac{1}{\ell !} \mathfrak{m}_{\ell}(\Gamma, \cdots, \Gamma) .
$$

It was shown in $[\mathrm{Fu}]$ (or $[\mathrm{FOOO}]$ for the $A_{\infty}$ case) that the new coderivation

$$
\delta^{\Gamma}=\sum_{\ell=0}^{\infty} \widehat{\mathfrak{m}}_{\ell}^{\Gamma}
$$

satisfies the $L_{\infty}$-relation $\delta^{\Gamma} \delta^{\Gamma}=0$ and so defines a weak $L_{\infty}$-algebra in general. By definition, $\Gamma$ satisfying (11.1) is equivalent to $\mathfrak{m}_{0}^{\Gamma}=0$. Therefore (11.1) is precisely the condition for this gauge changed (weak) $L_{\infty^{-}}$-structure to define a strong $L_{\infty^{-}}$ structure, and is the Maurer-Cartan equation for the deformation problem of the corresponding presymplectic structure $(Y, \omega)$ as well.

Now, we study (11.1) inductively over the degrees of the terms in the formal power series. Let and $\Gamma \in \Omega^{1}(\mathcal{F})$ and

$$
\Gamma=\sum_{k=1}^{\infty} \varepsilon^{k} \Gamma_{k}
$$

We fix a class $\alpha \in H^{1}(Y, \omega)$ and attempt to find $\Gamma$ that satisfies (11.1) in the formal power series and that $d_{\mathcal{F}}\left(\Gamma_{1}\right)=0$ and $\left[\Gamma_{1}\right]=\alpha \in H^{1}(Y, \omega)$.

For the sake of convenience, we will call $k$ the order of the formal power series. Obviously the lowest order term of (11.1) is

$$
\mathfrak{m}_{1}\left(\Gamma_{1}\right)=d_{\mathcal{F}}\left(\Gamma_{1}\right)=0
$$

whose solution we assume is given in the class $\alpha \in H^{1}(Y, \omega)$. Given $\Gamma_{1}$, the equation of the next order is

$$
\mathfrak{m}_{1}\left(\Gamma_{2}\right)+\frac{1}{2} \mathfrak{m}_{2}\left(\Gamma_{1}, \Gamma_{1}\right)=0 .
$$

From the $L_{\infty}$-relation, we know that $\mathfrak{m}_{1}$ is a derivation with respect to $\mathfrak{m}_{2}$. Therefore we have

$$
d_{\mathcal{F}}\left(\mathfrak{m}_{2}\left(\Gamma_{1}, \Gamma_{1}\right)\right)=0 \quad \text { in } \Omega^{\bullet}(\mathcal{F})
$$

since $d_{\mathcal{F}}\left(\Gamma_{1}\right)=0$. If we assume $\left[\mathfrak{m}_{2}\left(\Gamma_{1}, \Gamma_{1}\right)\right]=0$ in $H^{2}(Y, \omega)$, then there exists $\Gamma_{2}$ such that

$$
\mathfrak{m}_{1}\left(\Gamma_{2}\right)+\frac{1}{2} \mathfrak{m}_{2}\left(\Gamma_{1}, \Gamma_{1}\right)=0 .
$$

We set $\Gamma^{2}=\Gamma_{1}+\Gamma_{2}$ which will then solve (11.1) up to the order of 2 . We can repeat this process inductively over the degree $k$ to produce a solution

$$
\Gamma^{k}=\sum_{i=0}^{k} \varepsilon^{i} \Gamma_{i}
$$

up to the order of $k$ and then take the limit

$$
\Gamma=\lim _{k \rightarrow \infty} \Gamma^{k}=\sum_{\ell=1}^{\infty} \varepsilon^{\ell} \Gamma_{\ell}
$$


provided the obstruction class vanishes in each step. We remark that all the obstruction classes lie in the second cohomology $H^{2}(Y, \omega)$. The limit exists as a formal power series (or converges in the non-Archimedean topology induced by the degrees of $\left.\varepsilon^{k}\right)$. This proves the following general theorem.

Theorem 11.2. Let $\mathcal{F}$ be the null foliation of $(Y, \omega)$ and $\mathfrak{l}=\oplus_{\ell=1}^{n-k} \mathfrak{l}$ be the associated complex. Suppose that $H^{2}(Y, \omega)=\{0\}$, i.e, any $\mathcal{F}$-closed two form is $\mathcal{F}$-exact. Then for any given class $\alpha \in H^{1}(Y, \omega)$, 11.1) has a solution $\Gamma=\sum_{k=1}^{\infty} \varepsilon^{k} \Gamma_{k}$ such that $d_{\mathcal{F}}\left(\Gamma_{1}\right)=0$ and $\left[\Gamma_{1}\right]=\alpha \in H^{1}(Y, \omega)$. In other words, the formal moduli problem is unobstructed.

Question 11.2. Is it the case that whenever the formal moduli problem is unobstructed, the corresponding $C^{\infty}$ moduli problem is unobstructed? In other words, does the formal power series obtained in the unobstructed case converge?

We will investigate this question elsewhere.

Definition 11.3. A pre-symplectic manifold $(Y, \omega)$ is called unobstructed (resp. formally unobstructed) if the corresponding moduli problem (resp. formal moduli problem) is unobstructed. Otherwise it is called obstructed (resp. formally obstructed).

Corollary 11.3. Let $(Y, \omega)$ have the nullity 1, i.e., rank $E^{*}=1$. The moduli problem is unobstructed.

In fact, it is easy to see that in this hypersurface case, the genuine $C^{\infty}$ deformation problem is also unobstructed. Therefore the first non-trivial case will be the one $(Y, \omega)$ with nullity 2 .

We first provide a simple criterion for non-solvability of the Maurer-Cartan equation. We denote by

$$
Z^{\ell}(Y, \omega):=Z^{\ell}(\mathcal{F}) \subset \Omega^{\ell}(\mathcal{F})
$$

the set of $d_{\mathcal{F}}$-closed $\ell$-forms (respectively by $B^{\ell}(Y, \omega)$ the set of $d_{\mathcal{F}}$-exact one forms. Since $d_{\mathcal{F}}=\mathfrak{m}_{1}$ is the derivation of $\mathfrak{m}_{2}$, the bilinear map

$$
\mathfrak{l}^{1}=\Omega^{1}(\mathcal{F}) \rightarrow \mathfrak{l}^{2}=\Omega^{2}(\mathcal{F}) ; \Gamma_{1} \rightarrow \mathfrak{m}_{2}\left(\Gamma_{1}, \Gamma_{1}\right)
$$

canonically induces the map

$$
K r: H^{1}(Y, \omega) \rightarrow H^{2}(Y, \omega) ;\left[\Gamma_{1}\right] \rightarrow\left[\mathfrak{m}_{2}\left(\Gamma_{1}, \Gamma_{1}\right)\right],
$$

where [.] is the cohomology class associated to the given $d_{\mathcal{F}}$-closed form. This is a version of the Kuranishi map for this deformation problem which serves as the primary obstruction to the deformation. The pairing

$$
H^{1}(Y, \omega) \otimes H^{1}(Y, \omega) \rightarrow H^{2}(Y, \omega) ; \quad\left([\Gamma],\left[\Gamma^{\prime}\right]\right) \mapsto\left[\mathfrak{m}_{2}\left(\Gamma, \Gamma^{\prime}\right)\right]
$$

is a special case of the so-called Gerstenhaber bracket [Ge].

Theorem 11.4. Let $\alpha \in H^{1}(Y, \omega)$ such that $\operatorname{Kr}(\alpha) \neq 0$ in $H^{2}(Y, \omega)$. Then there is no solution

$$
\Gamma=\sum_{\ell=1}^{\infty} \varepsilon^{\ell} \Gamma_{\ell}
$$

for [11.1) with $\left[\Gamma_{1}\right]=\alpha$. In particular, there is no smooth isotopy $Y_{t} \subset(X, \omega)$ of coisotropic embeddings with

$$
Y_{0}=Y,\left.\quad \frac{d}{d t}\right|_{t=0} Y_{t}=\Gamma_{1} .
$$


One particular case is worth of mentioning

Corollary 11.5. Suppose that all $\mathfrak{m}_{k}=0$ for $k \geq 3$. Then $\alpha \in H^{1}(Y, \omega)$ is formally unobstructed if and only if $\operatorname{Kr}(\alpha)=0$.

Example 11.4. We will analyze the example studied by M. Zambon [Za] in the light of Theorem 11.4 This example illustrates that the space of $C^{1}$-close coisotropic submanifolds modulo the Hamiltonian isotopy is not smooth at the Hamiltonian isotopy class of the torus. Let $(Y, \omega)$ be the standard 4-torus $T^{4}=$ $\mathbb{R}^{4} / \mathbb{Z}^{4}$ with coordinates $\left(y^{1}, y^{2}, q^{1}, q^{2}\right)$ with the closed two form

$$
\omega_{Y}=d y^{1} \wedge d y^{2} \text {. }
$$

Note that the null foliation is provided by the 2-tori

$$
\left\{y^{1}=\text { const, } y^{2}=\text { const }\right\},
$$

and it also carries the transverse foliation given by

$$
\left\{q^{1}=\text { const, } q^{2}=\text { const }\right\} .
$$

The canonical symplectic thickening is given by

$$
\begin{aligned}
E^{*} & =T^{4} \times \mathbb{R}^{2}=T^{2} \times T^{*}\left(T^{2}\right), \\
\omega & =d y^{1} \wedge d y^{2}+\left(d q^{1} \wedge d p^{1}+d q^{2} \wedge d p^{2}\right),
\end{aligned}
$$

where $p^{1}, p^{2}$ are the canonical conjugate coordinates of $q^{1}, q^{2}$. It follows that the transverse curvature $F \equiv 0$ and so all $\mathfrak{m}_{\ell}=0$ for $\ell \geq 3$ and the Maurer-Cartan equation (11.1) becomes the quadratic equation

$$
d_{\mathcal{F}}(\Gamma)+\frac{1}{2}\{\Gamma, \Gamma\}=0
$$

where $\{\cdot, \cdot\}=\mathfrak{m}_{2}$ given by the formula in (11.6) below. In particular we have

$$
\int_{T^{2}}\{\Gamma, \Gamma\}=0
$$

for any solution $\Gamma$ of (11.4). It is easy to compute

$$
\begin{aligned}
& H^{0}(Y, \omega) \cong C^{\infty}\left(T^{2}\right), \\
& H^{1}(Y, \omega) \cong C^{\infty}\left(T^{2}\right)\left\{\theta^{1}, \theta^{2}\right\} \\
& H^{2}(Y, \omega) \cong C^{\infty}\left(T^{2}\right)\left\{\theta^{1} \wedge \theta^{2}\right\}
\end{aligned}
$$

where $\theta^{i}=\left[d q^{i}\right] \in H^{1}(Y, \omega)$. We consider a one form $\Gamma=a_{1}(y, q) d q^{1}+a_{2}(y, q) d q^{2}$ that is $d_{\mathcal{F}}$-closed, i.e., satisfies

$$
\frac{\partial a_{2}}{\partial q^{1}}-\frac{\partial a_{1}}{\partial q^{2}}=0
$$

The map $K r: H^{1}(Y, \omega) \rightarrow H^{2}(Y, \omega)$ is induced by the bilinear map

$$
\Gamma \rightarrow \mathfrak{m}_{2}(\Gamma, \Gamma),
$$

which can be written in coordinates as

$$
a_{1}(y, q) d q^{1}+a_{2}(y, q) d q^{2} \rightarrow\left\{a_{1}, a_{2}\right\}_{y} d q^{1} \wedge d q^{2},
$$

where the bracket is defined by

$$
\left\{a_{1}, a_{2}\right\}_{y}=\left(\left(\frac{\partial a_{1}}{\partial y^{2}} \frac{\partial a_{2}}{\partial y^{1}}\right)-\left(\frac{\partial a_{2}}{\partial y^{2}} \frac{\partial a_{1}}{\partial y^{1}}\right)\right) .
$$


Therefore any infinitesimal deformation $\Gamma$ with the non-vanishing integration over the fiber $\Sigma_{\left(y^{1}, y^{2}\right)}=T^{2}$

$$
\int_{\Sigma_{\left(y^{1}, y^{2}\right)}}\left\{a_{1}, a_{2}\right\}_{y} d q^{1} \wedge d q^{2} \neq 0
$$

will be obstructed. For example, one can take

$$
\Gamma=\sin \left(2 \pi y^{1}\right) d q^{1}+\sin \left(2 \pi y^{2}\right) d q^{2}
$$

which is the example Zambon looked at. In this case, we have

$$
\int\left\{a_{1}, a_{2}\right\}_{y} d q^{1} \wedge d q^{2}=-4 \pi^{2} \cos 2 \pi y^{2} \cos 2 \pi y^{1} \not \equiv 0 .
$$

In the next section, we study a more non-trivial example where the structure of null foliations becomes more complicated.

\section{An eXAmple}

In this section, we analyze one parameter family of examples $\left(Y_{\alpha}, \omega_{\alpha}\right)$ from the mechanics of harmonic oscillator. From the analysis of this example, it is manifest that the deformation problem of coisotropic submanifolds is closely tied to the geometry and dynamics of the null foliation. A systematic study of geometry of coisotropic submanifolds in terms of geometry of the foliation theory will be carried out elsewhere.

Consider the harmonic oscillator

$$
H=\frac{1}{2}\left(|Q|^{2}+|P|^{2}\right)
$$

on the phase space $T^{*} \mathbb{R}^{3} \cong \mathbb{R}^{6}$ where $Q=\left(Q^{1}, Q^{2}, Q^{3}\right)$ and $P=\left(P^{1}, P^{2}, P^{3}\right)$ are the position and momentum coordinates. We can write

$$
H=H_{1}+H_{2}+H_{3},
$$

where $H_{i}$ are the one dimensional harmonic oscillator Hamiltonians

$$
H_{i}=\frac{1}{2}\left(\left(Q^{i}\right)^{2}+\left(P^{i}\right)^{2}\right), \quad i=1,2,3 .
$$

It follows that $\left\{H, H_{i}\right\}=\left\{H_{i}, H_{j}\right\}=0$ where $\{\cdot, \cdot\}$ is the canonical Poisson bracket. We fix two constants $\alpha, \beta>0$ such that

$$
\alpha>1, \quad 0<\beta<\frac{1}{2},
$$

and consider the submanifold $Y_{\alpha, \beta} \subset S^{5} \subset \mathbb{R}^{6}$ defined by

$$
Y_{\alpha, \beta}=\left\{(q, p) \in \mathbb{R}^{6} \mid H(q, p)=\frac{1}{2},\left(H_{1}+\alpha H_{2}\right)(q, p)=\beta\right\} .
$$

It is easy to see that these provide two parameter family of smooth coisotropic submanifolds of $\mathbb{R}^{6}$ whose images are all contained in the unit sphere $H^{-1}(1 / 2)$. To simplify the discussion, we fix $\beta=\frac{1}{4}$ and denote

$$
Y_{\alpha}:=Y_{\alpha, 1 / 4}, \quad H_{\alpha}:=H_{1}+\alpha H_{2} .
$$

It is straightforward to check that the Hamiltonian vector fields $X_{H}$ and $X_{H_{\alpha}}$ are linearly independent everywhere on $Y_{\alpha}$ and so the characteristic distribution is given by

$$
E=\operatorname{span}_{\mathbb{R}}\left\{X_{H}, X_{H_{\alpha}}\right\}
$$


which is a trivial bundle. In particular all leaves are orientable. We recall

$$
Y_{\alpha}=\left\{\left.(Q, P) \in \mathbb{R}^{6}|| Q\right|^{2}+|P|^{2}=1, H_{1}+\alpha H_{2}=\frac{1}{4}\right\} .
$$

On $Y_{\alpha}$, we derive

$$
\begin{aligned}
& H_{1}=\frac{1}{\alpha-1}\left(\frac{2 \alpha-1}{4}-\alpha H_{3}\right), \\
& H_{2}=\frac{1}{\alpha-1}\left(H_{3}-\frac{1}{4}\right) .
\end{aligned}
$$

Since $H_{1}, H_{2} \geq 0$, we have obtained the bound

$$
\frac{1}{4} \leq H_{3} \leq \frac{2 \alpha-1}{4 \alpha}
$$

We will denote by $\ell_{i}$ the value of the corresponding $H_{i}$. For $\frac{1}{4}<\ell_{3}<\frac{2 \alpha-1}{4 \alpha}, H_{3}$ are regular on $Y_{\alpha}$ and $H_{3}^{-1}\left(\ell_{3}\right)$ is a 3 -torus

$$
H_{3}^{-1}\left(\ell_{3}\right)=S^{1}\left(\sqrt{2 \ell_{1}}\right) \times S^{1}\left(\sqrt{2 \ell_{2}}\right) \times S^{1}\left(\sqrt{2 \ell_{3}}\right),
$$

where $\ell_{i}, i=1,2$ are given by the formula (12.1) for a given $\ell_{3}$. It follows that leaves of the null foliation on the open subset $H_{3}^{-1}\left(\frac{1}{4}, \frac{2 \alpha-1}{4 \alpha}\right) \subset Y_{\alpha}$ are generated by

$$
\{(1,1,1),(1, \alpha, 0)\}
$$

in the rectangularpid $[0,2 \pi] \times[0,2 \pi] \times[0,2 \pi]$. Note that this family of lines is uniquely determined by $\alpha$ and $\ell_{3}$.

When $\alpha$ is rational, then all the leaves are compact. When $\alpha$ is irrational, all the leaves are non-compact. They are all immersions of $\mathbb{R} \times S^{1}$ are dense in the three torus $H_{3}^{-1}\left(\ell_{3}\right), \ell_{3} \neq \frac{1}{4}, \frac{2 \alpha-1}{4 \alpha}$ which are invariant under the Hopf action of $S^{1}$ on $Y_{\alpha} \subset S^{5} \subset \mathbb{C}^{3}$.

When $\ell_{3}=\frac{1}{4}$, we have $\ell_{2}=0$ and $\ell_{1}=\frac{1}{4}$. Hence $\left\{(Q, P) \in Y_{\alpha} \mid \ell_{3}=\frac{1}{4}\right\}$ consists of the unique leaf which is nothing but

$$
S^{1}\left(\frac{1}{\sqrt{2}}\right) \times\{(0,0)\} \times S^{1}\left(\frac{1}{\sqrt{2}}\right) .
$$

Similarly when $\ell_{3}=\frac{2 \alpha-1}{4 \alpha}$, we have $\ell_{1}=0$ and $\ell_{2}=\frac{1}{4 \alpha}$ and hence $\left\{(Q, P) \in Y_{\alpha} \mid\right.$ $\left.\ell_{3}=\frac{2 \alpha-1}{4 \alpha}\right\}$ is the torus

$$
\{(0,0)\} \times S^{1}\left(\frac{1}{\sqrt{4 \alpha}}\right) \times S^{1}\left(\sqrt{\frac{2 \alpha-1}{4 \alpha}}\right) .
$$

Now we compute the transverse curvature of the leaves. For this we consider the canonical splitting provided by the complex structure on $\mathbb{R}^{6} \cong \mathbb{C}^{3}$. In other words, we choose the splitting $T Y=G \oplus E$ with $G$ given by

$$
G=(E \oplus J E)^{\perp},
$$

where $\perp$ is the Euclidean orthogonal complement and $J$ denote the standard complex structure on $\mathbb{C}^{3}$. We choose coordinates $\left(y^{1}, y^{2}, q^{1}, q^{2}\right)$ of $Y_{\alpha}$ by the functions

$$
\begin{aligned}
y^{1} & =\alpha \theta_{1}-\theta_{2}-\frac{1+\alpha^{2}}{\alpha} \theta_{3}, \\
y^{2} & =H_{3}, \\
q^{1} & =\theta_{3}, \\
q^{2} & =\theta_{1}+\alpha \theta_{2}+\left(1+\alpha^{2}\right) \theta_{3} .
\end{aligned}
$$


Here $\left(r_{1}, r_{2}, r_{3}, \theta_{1}, \theta_{2}, \theta_{3}\right)$ is the polar coordinates of $\mathbb{R}^{6}$ and we have chosen $y^{1}, y^{2}$ so that the leaves of the null foliation are given by

$$
\left\{y^{1}=\text { const, } y^{2}=\text { const }\right\} \text {. }
$$

A straightforward calculation leads to

$$
H_{1}=\frac{1}{\alpha-1}\left(\frac{2 \alpha-1}{4}-\alpha y^{2}\right), \quad H_{2}=\frac{1}{\alpha-1}\left(y^{2}-\frac{1}{4}\right), \quad H_{3}=y^{2} .
$$

We derive

$$
\begin{aligned}
& \theta_{1}=q^{1}+\frac{q^{2}+\alpha y^{1}}{1+\alpha^{2}}, \\
& \theta_{2}=q^{1}+\frac{\alpha q^{2}-y^{1}}{1+\alpha^{2}}, \\
& \theta_{3}=q^{1} .
\end{aligned}
$$

Therefore $Y_{\alpha}$ is parameterized by

$$
\left(r_{1} e^{i\left(q^{1}+\frac{q^{2}+\alpha y^{1}}{1+\alpha^{2}}\right)}, r_{2} e^{i\left(q^{1}+\frac{\alpha q^{2}-y^{1}}{1+\alpha^{2}}\right)}, r_{3} e^{i q^{1}}\right)
$$

in this coordinates, where we note $r_{i}=\sqrt{2 L_{i}}$. From this, we derive

$$
\begin{aligned}
\frac{\partial}{\partial y^{1}} & =\frac{\alpha}{1+\alpha^{2}} \frac{\partial}{\partial \theta_{1}}-\frac{1}{1+\alpha^{2}} \frac{\partial}{\partial \theta_{2}}=\frac{1}{1+\alpha^{2}} X_{\alpha H_{1}-H_{2}}, \\
\frac{\partial}{\partial y^{2}} & =-\frac{\alpha}{2(\alpha-1)} \frac{1}{r_{1}} \frac{\partial}{\partial r_{1}}+\frac{1}{2(\alpha-1)} \frac{1}{r_{2}} \frac{\partial}{\partial r_{2}}+\frac{1}{2 r_{3}} \frac{\partial}{\partial r_{3}}, \\
\frac{\partial}{\partial q^{1}} & =\frac{\partial}{\partial \theta_{1}}+\frac{\partial}{\partial \theta_{2}}+\frac{\partial}{\partial \theta_{3}}=X_{H}, \\
\frac{\partial}{\partial q^{2}} & =\frac{1}{1+\alpha^{2}} \frac{\partial}{\partial \theta_{1}}+\frac{\alpha}{1+\alpha^{2}} \frac{\partial}{\partial \theta_{2}}=\frac{1}{1+\alpha^{2}} X_{H_{\alpha}} .
\end{aligned}
$$

We consider the orthogonal splitting $T Y=G \oplus E$ with respect the induced metric on $Y_{\alpha}$ from the Euclidean metric on $\mathbb{R}^{6}$. A calculation shows

$$
\begin{aligned}
G & =\operatorname{span}_{\mathbb{R}}\left\{-\frac{\alpha}{r_{1}^{2}} \frac{\partial}{\partial \theta_{1}}+\frac{1}{r_{2}^{2}} \frac{\partial}{\partial \theta_{2}}, \frac{\partial}{\partial y^{2}}\right\} \\
& =\operatorname{span}_{\mathbb{R}}\left\{-\frac{\alpha}{H_{1}} \frac{\partial}{\partial \theta_{1}}+\frac{1}{H_{2}} \frac{\partial}{\partial \theta_{2}}, \frac{\partial}{\partial y^{2}}\right\} .
\end{aligned}
$$

A straightforward but lengthy calculation gives

$$
G=\operatorname{span}_{\mathbb{R}}\left\{e_{1}, e_{2}\right\}
$$

where

$$
\begin{aligned}
& e_{1}=\frac{\partial}{\partial y^{1}}-\frac{\alpha\left(H_{1}-H_{2}\right)}{\alpha^{2} H_{2}+H_{1}} \frac{\partial}{\partial q^{2}}, \\
& e_{2}=\frac{\partial}{\partial y^{2}} .
\end{aligned}
$$

Hence we have the matrix $\left(R_{i}^{\alpha}\right)$ given by

$$
R_{2}^{1}=0, \quad R_{2}^{2}=-\frac{\alpha\left(H_{1}-H_{2}\right)}{\alpha^{2} H_{2}+H_{1}}, \quad R_{1}^{1}=R_{1}^{2}=0 .
$$


Noting that $H_{i}$ 's are functions of $y^{2}$ alone in (12.1), it follows from 4.8 that $F_{i j}^{\alpha} \equiv 0$, which can be also seen from the observation that the chosen splitting $G$ is also integrable. This proves

$$
\mathfrak{m}_{k}=0 \text { for all } k \geq 3,
$$

and the corresponding homotopy algebra reduces a differential graded Lie algebra. A straightforward calculation also shows

$$
\left.\omega\right|_{Y_{\alpha}}=\frac{1}{2(\alpha-1)} d y^{1} \wedge d y^{2}
$$

on $Y_{\alpha} \backslash H_{3}^{-1}\left(\frac{1}{4}\right) \cup H_{3}^{-1}\left(\frac{2 \alpha-1}{4 \alpha}\right)$.

Finally we study the Kuranishi map

$$
K r: H^{1}\left(Y_{\alpha}, \omega_{\alpha}\right) \rightarrow H^{2}\left(Y_{\alpha}, \omega_{\alpha}\right) ; \quad[\Gamma] \rightarrow\left[\mathfrak{m}_{2}(\Gamma, \Gamma)\right],
$$

where $\Gamma$ is $d_{\mathcal{F}}$-closed one form. We first need to characterize the set of $d_{\mathcal{F}}$-closed one forms. Recalling that $E=\operatorname{span}_{\mathbb{R}}\left\{X_{H}, X_{H_{\alpha}}\right\}$, we represent $E^{*}=\left\{f_{1}^{*}, f_{2}^{*}\right\}$ where $\left\{f_{1}^{*}, f_{2}^{*}\right\}$ is the dual frame of $\left\{X_{H}, X_{H_{\alpha}}\right\}$. Let

$$
\Gamma=A f_{1}^{*}+B f_{2}^{*}
$$

be a one form in $\Omega^{1}(\mathcal{F})$ where $A, B$ are globally defined function on $Y_{\alpha}$. From the definition of $d_{\mathcal{F}}$, we have

$$
d_{\mathcal{F}}(\Gamma)=\left(X_{H}(B)-X_{H_{\alpha}}(A)\right) f_{1}^{*} \wedge f_{2}^{*} .
$$

Therefore $\Gamma$ is $d_{\mathcal{F}}$-closed if and only if

$$
X_{H}(B)=X_{H_{\alpha}}(A) .
$$

On the other hand, for any smooth function $C \in C^{\infty}\left(Y_{\alpha}\right)$, we have

$$
d_{\mathcal{F}}(C)=X_{H}[C] f_{1}^{*}+X_{H_{\alpha}}[C] f_{2}^{*} .
$$

We will compute $H^{1}(Y, \omega)$ and $H^{2}(Y, \omega)$ and study the Kuranishi map

$$
K r: H^{1}(Y, \omega) \rightarrow H^{2}(Y, \omega) .
$$

12.1. Symplectic reduction and integration over fibers. We first apply the symplectic reduction with respect to the Hopf action of $S^{1}$ which induces the following commutative diagram

$$
\begin{aligned}
& Y_{\alpha} \quad \hookrightarrow \quad S^{5} \subset \mathbb{C}^{3} \backslash\{0\} \\
& \downarrow \pi_{1} \quad \downarrow \pi_{1} \\
& \tilde{Y}_{\alpha}=Y_{\alpha} / S^{1} \hookrightarrow \quad \mathbb{C} P^{2}
\end{aligned}
$$

We will denote by $\widetilde{\omega}$ the reduced symplectic form on $H^{-1}\left(\frac{1}{2}\right) / S^{1}=S^{5} / S^{1} \cong \mathbb{C} P^{2}$ which is nothing but the standard Fubini-Study form, and by $\widetilde{\omega}_{\alpha}$ the induced presymplectic form on $\tilde{Y}_{\alpha} \subset \mathbb{C} P^{2}$. Since $H_{\alpha}$ is invariant under the Hopf action, it projects down to a function $\widetilde{H}_{\alpha}$ on $\widetilde{Y}_{\alpha}$.

$\widetilde{Y}_{\alpha} \subset \mathbb{C} P^{2}$ is a hypersurface for which the induced null foliation $\widetilde{\mathcal{F}}$ on $\widetilde{Y}_{\alpha}$ are given by $\Sigma / S^{1}$ for each $\Sigma$ is a leaf of $\mathcal{F}$. In fact, we have the obvious one-one correspondence

$$
Y_{\alpha} / \sim \cong \tilde{Y}_{\alpha} / \sim
$$

Furthermore $d_{\widetilde{\mathcal{F}}}$ is given by

$$
d_{\widetilde{\mathcal{F}}}(f)=X_{\widetilde{H}_{\alpha}}[f] \widetilde{f}_{2}^{*}
$$


for $f \in C^{\infty}\left(\widetilde{Y}_{\alpha}\right)$ where $\tilde{f}_{2}^{*}$ is the push-forward of $f_{2}^{*}$ to $\tilde{Y}_{\alpha}$. We denote by

$$
\left(\pi_{1}\right)_{*}: \Omega^{1}\left(Y_{\alpha}, \omega_{\alpha}\right) \rightarrow C^{\infty}\left(\widetilde{Y}_{\alpha}\right)=\Omega^{0}\left(\widetilde{Y}_{\alpha}, \widetilde{\omega}_{\alpha}\right)
$$

the integration over fibers defined by

$$
\left(\pi_{1}\right)_{*}\left(A f_{1}^{*}+B f_{2}^{*}\right)(y)=\int_{\pi_{1}^{-1}(y)} A f_{1}^{*} .
$$

It is easy to check the identity

$$
d_{\widetilde{\mathcal{F}}} \circ\left(\pi_{1}\right)_{*}=\left(\pi_{1}\right)_{*} \circ d_{\mathcal{F}}
$$

and so $\left(\pi_{1}\right)_{*}$ induces a natural homomorphism

$$
\left(\pi_{1}\right)_{*}: H^{1}\left(Y_{\alpha}, \omega_{\alpha}\right) \rightarrow C^{\infty}\left(\widetilde{Y}_{\alpha}\right)
$$

Similarly we define

$$
\left(\pi_{1}\right)_{*}: \Omega^{2}\left(Y_{\alpha}, \omega_{\alpha}\right) \rightarrow \Omega^{1}\left(\widetilde{Y}_{\alpha}, \widetilde{\omega}_{\alpha}\right)
$$

by

$$
\left(\pi_{1}\right)_{*}\left(D \cdot f_{1}^{*} \wedge f_{2}^{*}\right)=\left(\int_{\pi^{-1}(y)} D f_{1}^{*}\right) \tilde{f}_{2}^{*}
$$

which again intertwines $d_{\mathcal{F}}$ and $d_{\widetilde{\mathcal{F}}}$ and so induces the homomorphism

$$
\left(\pi_{1}\right)_{*}: H^{2}\left(Y_{\alpha}, \omega_{\alpha}\right) \rightarrow H^{1}\left(\widetilde{Y}_{\alpha}, \widetilde{\omega}_{\alpha}\right) .
$$

Since the spaces of leaves $Y_{\alpha} / \sim$ and $\widetilde{Y}_{\alpha} / \sim$ are isomorphic, it is easy to check that

$$
\left(\pi_{1}\right)_{*}: H^{2}\left(Y_{\alpha}, \omega_{\alpha}\right) \rightarrow H^{1}\left(\widetilde{Y}_{\alpha}, \widetilde{\omega}_{\alpha}\right)
$$

is an isomorphism. We next quote the following general theorem by Haefliger [Ha]. Here $\Omega_{c}^{\bullet}(\operatorname{Tr} \mathcal{F})$ is the topological differential graded vector space of forms on $T / H$, where $H$ is the holonomy pseudogroup induced on a complete transversal submanifold $T$. Since we will not use the theorem except the presence of the isomorphism (12.12), we refer readers to [Ha] for more detailed explanation.

Theorem 12.1. [Theorem 3.1, Ha] Let $\mathcal{F}$ be a foliation on $X$ with leaves of dimension $p$, and assume that the tangent bundle to the leaves is oriented. Then there is a continuous open surjective linear map

$$
\int_{\mathcal{F}}: \Omega_{c}^{p+k}(X) \rightarrow \Omega_{c}^{k}(\operatorname{Tr} \mathcal{F})
$$

which commutes with $d_{\mathcal{F}}$. And it induces an isomorphism

$$
\int_{\mathcal{F}}: H^{p}(\mathcal{F}) \rightarrow \Omega^{0}(\operatorname{Tr} \mathcal{F})
$$

Since we have the isomorphism

$$
\Omega^{0}(\operatorname{Tr} \mathcal{F}) \cong \Omega^{0}(\operatorname{Tr} \widetilde{\mathcal{F}})
$$

which obviously follows from the fact that the spaces of leaves $Y_{\alpha} / \sim$ and $\widetilde{Y}_{\alpha} / \sim$ are isomorphic,

$$
\pi_{*}: H^{2}\left(Y_{\alpha}, \omega_{\alpha}\right) \rightarrow H^{1}\left(\widetilde{Y}_{\alpha}, \widetilde{\omega}_{\alpha}\right)
$$

is an isomorphism. It is also known [Ha] that $\Omega^{0}(\operatorname{Tr} \mathcal{F})$ is naturally isomorphic to $C^{\infty}(Y / \sim)$ when the leaf space $Y / \sim$ is an orbifold. One can also prove these statements directly without referring to the above result from [Ha]. We did this to 
illustrate a connection of the current deformation problem to the foliation theory. A more systematic study in this regard will be carried out elsewhere in the future.

From now on we treat two cases, $\alpha$ rational and irrational, separately. We will postpone the study of irrational case to a future work.

12.2. The rational case. We first note that the foliation $\mathcal{F}$ is generated by the closed one forms

$$
\begin{aligned}
d y^{1} & =\alpha d \theta_{1}-d \theta_{2}-\frac{1+\alpha_{2}}{\alpha} d \theta_{3} \\
d y^{2} & =d H_{3} .
\end{aligned}
$$

Both are globally well-defined and the period group is given by

$$
P:=\operatorname{span}_{\mathbb{Z}}\left\{d y^{1}\left(H_{1}\left(Y_{\alpha}, \mathbb{Z}\right)\right), d y^{2}\left(H_{1}\left(Y_{\alpha}, \mathbb{Z}\right)\right)\right\}=\operatorname{span}_{\mathbb{Z}}\left\{\alpha,-\frac{1+\alpha^{2}}{\alpha}\right\} .
$$

Suppose that $\alpha$ is rational and $\alpha=\frac{p}{q}$ with relatively prime integers $p, q$. The period group $P$ of $\mathcal{F}$ is generated by

$$
\left\{\frac{p}{q},-\left(\frac{p}{q}+\frac{q}{p}\right)\right\}
$$

and so

If we denote

$$
P=\operatorname{span}_{\mathbb{Z}}\left\{\frac{\operatorname{gcd}\left\{p^{2},-\left(p^{2}+q^{2}\right)\right\}}{q p}\right\}
$$

$$
\frac{L}{M}=\frac{\operatorname{gcd}\left\{p^{2},-p q,-q^{2}\right\}}{q p}
$$

with $L, M$ relatively prime to each other, then the local holonomy group is trivial except the two leaves $H_{3}^{-1}\left(\frac{1}{4}\right)$ and $H_{3}^{-1}\left(\frac{2 \alpha-1}{4 \alpha}\right)$ at which the holonomy groups are finite cyclic groups.

It turns out that the leaf space $Y_{\alpha} / \sim$ is a compact Hausdorff symplectic orbifold induced by the canonical transverse symplectic form. There are two orbifold points mentioned above. We denote them by $z^{-}, z^{+} \in Y_{\alpha} / \sim$ respectively. See [Sa] for the definition of orbifolds (or V-manifolds) and [We3] for the definition of symplectic orbifolds (or symplectic V-manifolds).

Now we describe the leaf space more precisely. It remains to study local structure near the two orbifold points $z_{ \pm}$. Around the leaf $H_{3}^{-1}\left(\frac{1}{4}\right)$, we choose coordinates

$$
\left(H_{2}, \theta_{2}, q^{1}, q^{2}\right)
$$

where $q^{1}, q^{2}$ are the ones as before. It follows from (12.1)

$$
\begin{aligned}
& y^{1}=\alpha q^{2}+\left(1+\alpha^{2}\right) q^{1}-\left(1+\alpha^{2}\right) \theta_{2} \\
& y^{2}=(\alpha-1) H_{2}+\frac{3}{4}
\end{aligned}
$$

and so

$$
\frac{\partial}{\partial H_{2}}=(\alpha-1) \frac{\partial}{\partial y^{2}}, \frac{\partial}{\partial \theta_{2}}=-\left(1+\alpha^{2}\right) \frac{\partial}{\partial y^{1}} .
$$

Substituting this into (12.8), we derive

$$
\left.\omega\right|_{Y_{\alpha}}=\left(1+\alpha^{2}\right) d H_{2} \wedge d \theta_{2}=\left(1+\alpha^{2}\right) r_{2} d r_{2} \wedge d \theta_{2} .
$$


Recall that $\left(y^{1}, y^{2}\right)$ parameterizes the leaf space around $z_{1}$ and two leaves parameterized by $\left(y^{1}, y^{2}\right)$ and $\left(Y^{1}, Y^{2}\right)$ coincide if and only if

$$
y^{2}=Y^{2}, \quad \text { and } y^{1} \equiv Y^{1} \quad \bmod 2 \pi .
$$

In the coordinates $\left(H_{2}, \theta_{2}\right)$, the latter condition is equivalent to

$$
-\left(1+\alpha^{2}\right) \theta_{2} \equiv-\left(1+\alpha^{2}\right) \Theta_{2} \quad \bmod 2 \pi \Longleftrightarrow \theta_{2} \equiv \Theta_{2} \quad \bmod \frac{2 \pi}{1+\alpha^{2}} .
$$

Therefore the local chart of $Y_{\alpha} / \sim$ is given by the non-flat cone

$$
\mathbb{R}^{2} / \mathbb{Z}_{M_{-}}
$$

if we denote

$$
\frac{1}{1+\alpha^{2}}=\frac{L_{-}}{M_{-}}
$$

with $L_{-}, M_{-}$relatively prime. Note that the symplectic form is certainly invariant under the linear action by $\mathbb{Z}_{M_{-}}$. Similar computation can be carried out at the leaf $z^{+}=H_{3}^{-1}\left(\frac{2 \alpha-1}{4 \alpha}\right)$ using the coordinates

$$
\left(H_{1}, \theta_{1}, q^{1}, q^{2}\right)
$$

and have the symplectic form

$$
\omega=\frac{1+\alpha^{2}}{2 \alpha^{2}} d H_{1} \wedge d \theta_{1}=\frac{1+\alpha^{2}}{\alpha^{2}} r_{1} d r_{1} \wedge d \theta_{1} .
$$

This time the holonomy group is $Z_{M_{+}}$where we write

$$
\frac{\alpha}{1+\alpha^{2}}=\frac{L_{+}}{M_{+}}
$$

for relatively prime integers $L_{+}, M_{+}$. It is easy to see that the $Y_{\alpha} / \sim$ is topologically sphere. We denote the corresponding symplectic orbifold by

$$
\left(S_{\alpha}^{2}, \omega_{\alpha}\right)
$$

In fact in the rational case, the flows of $H$ and $H_{\alpha}$ generate two circle actions which commute each other and so defines a torus action. The leaves of the null foliation are then just the orbits of this torus action and defines a fibration over $S_{\alpha}^{2}$. See (12.5). We denote by $C^{\infty}\left(S_{\alpha}^{2}\right)$ the set of smooth functions on the orbifold $S_{\alpha}^{2}$. Then we have

Proposition 12.2. [2nd Cohomology] When $\alpha>1$ is rational, we have

$$
H^{2}\left(Y_{\alpha}, \omega_{\alpha}\right) \cong C^{\infty}\left(S_{\alpha}^{2}\right)\left\{a^{1} \wedge a^{2}\right\} .
$$

Proof. This follows from (12.12)-12.13) and from the remark right after Theorem 12.1

We next analyze $H^{1}\left(Y_{\alpha}, \omega_{\alpha}\right)$. We consider an open covering of $S_{\alpha}^{2}=D^{+} \cup D^{-}$ where $D^{ \pm}$is a neighborhood of the two orbifold points $p^{ \pm}$such that $D_{\alpha}^{+} \cap D_{\alpha}^{-}$is diffeomorphic to $S^{1} \times(-\varepsilon, \varepsilon)$. We denote

$$
Y^{ \pm}=\pi^{-1}\left(D_{\alpha}^{ \pm}\right)
$$


which provides a covering $Y_{\alpha}=Y^{+} \cup Y^{-}$. We emphasize that both $D_{\alpha}^{ \pm}$are orbifolds diffeomorphic to $D^{2} / \mathbb{Z}_{M^{ \pm}}$respectively. We then consider the Mayer-Vietoris sequence

$$
\begin{aligned}
& \rightarrow H^{0}\left(Y^{+} \cap Y^{-}, \omega_{\alpha}\right) \stackrel{\delta^{0}}{\rightarrow} H^{1}\left(Y_{\alpha}, \omega_{\alpha}\right) \stackrel{\alpha^{1}}{\rightarrow} \\
& \stackrel{\alpha^{1}}{\rightarrow} H^{1}\left(Y^{+}, \omega_{\alpha}\right) \oplus H^{1}\left(Y^{-}, \omega_{\alpha}\right) \stackrel{\beta^{1}}{\rightarrow} H^{1}\left(Y^{+} \cap Y^{-}, \omega_{\alpha}\right) \stackrel{\delta^{1}}{\rightarrow} H^{2}\left(Y_{\alpha}, \omega_{\alpha}\right) \rightarrow
\end{aligned}
$$

From this exact sequence, we have derived

$$
H^{1}\left(Y_{\alpha}, \omega_{\alpha}\right) \cong \operatorname{im} \delta^{0} \oplus \operatorname{ker} \beta^{1}
$$

We now describe the two summands more explicitly. We note that the forms

$$
f_{1}^{*}, f_{2}^{*}
$$

are leafwise closed, and invariant under the torus action and also under the holonomy. We denote the corresponding cohomology class thereof by $a_{j}=\left[f_{j}^{*}\right] \in$ $H^{1}\left(Y_{\alpha}, \omega_{\alpha}\right)$. Then the above discussion gives rise to

$$
\begin{aligned}
H^{1}\left(Y^{+} \cap Y^{-}, \omega_{\alpha}\right) & \cong C^{\infty}\left(S^{1}\right)\left\{a^{1}, a^{2}\right\} \\
H^{1}\left(Y^{+}, \omega_{\alpha}\right) & \cong C^{\infty}\left(D_{\alpha}^{+}\right)\left\{a^{1}, a^{2}\right\} \\
H^{1}\left(Y^{-}, \omega_{\alpha}\right) & \cong C^{\infty}\left(D_{\alpha}^{-}\right)\left\{a^{1}, a^{2}\right\}
\end{aligned}
$$

Furthermore again from the above exact sequence, we have

$$
\operatorname{ker} \beta^{1} \cong H^{1}\left(Y^{+}, \omega_{\alpha}\right) \times_{H^{1}\left(Y^{+} \cap Y^{-}, \omega_{\alpha}\right)} H^{1}\left(Y^{-}, \omega_{\alpha}\right) \cong C^{\infty}\left(S_{\alpha}^{2}\right)\left\{a^{1}, a^{2}\right\}
$$

where the middle term is the obvious fiber product. This finishes the description of the first cohomology. We summarize our discussion into

Proposition 12.3. [1st Cohomology] Let $\alpha>1$ be rational. Then we have the isomorphism

$$
\begin{aligned}
H^{1}\left(Y_{\alpha}, \omega_{\alpha}\right) & \cong \operatorname{ker} \beta^{1} \oplus \operatorname{im} \delta^{0} \\
& \cong C^{\infty}\left(S_{\alpha}^{2}\right)\left\{a^{1}, a^{2}\right\} \oplus \operatorname{im} \delta^{0} .
\end{aligned}
$$

Next we study the Gerstenhaber bracket $[\cdot, \cdot]$ and the Kuranishi map

Proposition 12.4. [Gerstenhaber bracket and Kuranishi map] Under the isomorphisms in Theorem 12.2 and Theorem 12.3, the Gerstenhaber bracket is given by

$$
[\gamma, \eta]=0
$$

for all $\gamma \in H^{1}\left(Y_{\alpha}, \omega_{\alpha}\right)$ and $\eta \in \operatorname{im} \delta^{0}$ and

$$
\left[\gamma_{1}, \gamma_{2}\right]=\left(\left\{g_{1}, h_{2}\right\}_{\alpha} \circ \pi\right) a^{1} \wedge a^{2}
$$

where $\Gamma_{j}=g_{j} a^{1}+h_{j} a^{2}, \quad j=1,2$ and $g_{j}, h_{j} \in C^{\infty}\left(S_{\alpha}^{2}\right)$, and $\{\cdot, \cdot\}_{\alpha}$ is the Poisson bracket on $S_{\alpha}^{2}$. In particular, the Kuranishi map $K r$ is given by the formula

$$
\operatorname{Kr}(\Gamma)=\left(\{g, h\}_{\alpha} \circ \pi\right) a^{1} \wedge a^{2}
$$

for $\Gamma=g a^{1}+h a^{2}$. 
Proof. We first note that $\operatorname{ker} \alpha^{1}=\operatorname{im} \delta^{0}$ : any element $a$ lying in $\operatorname{im} \delta^{0} \subset H^{1}\left(Y, \omega_{\alpha}\right)$ is represented by a leafwise one form $\alpha$ determined by

$$
\alpha= \begin{cases}d g_{+} & \text {on } Y^{+} \\ d g_{-} & \text {on } Y^{-}\end{cases}
$$

such that $i_{+}^{*} g_{+}-i_{-}^{*} g_{-}=f$ for some leafwise constant function $f$ or equivalently for $f$ satisfying $d_{\mathcal{F}}(f)=0$ on $Y^{+} \cap Y^{-}$. Using this representation of an element from im $\delta^{0}$ and Theorem 9.3, which states that $d_{\mathcal{F}}$ is a derivation of $\{\cdot, \cdot\}_{\Pi}$ because $F_{\Pi} \equiv 0$, it is easy to verify (12.18).

For the proof of (12.19), we use the isomorphism (12.17) and the definition of the bracket $\{\cdot, \cdot\}_{\Pi}$, the details of which we omit. This finishes the proof.

Using this proposition, if we choose any smooth function $a, b \in C^{\infty}\left(S_{\alpha}^{2}\right)$ with $\{a, b\}_{S_{\alpha}^{2}} \neq 0$ and set $\Gamma=(a \circ \pi) f_{1}^{*}+(b \circ \pi) f_{2}^{*}$, then we derive

$$
\int_{\mathcal{F}}\{\Gamma, \Gamma\}_{\Pi}=\{a, b\}_{S_{\alpha}^{2}} \neq 0 .
$$

Here $\pi: Y_{\alpha} \rightarrow Y_{\alpha} / \sim$ is the obvious projection. Therefore the cohomology class $\alpha \in H^{1}(Y, \omega)$ is obstructed. We summarize the above discussion into the following theorem.

Theorem 12.5. Let $\alpha>1$ be rational. Then the coisotropic submanifold $Y_{\alpha} \subset \mathbb{R}^{6}$ or equivalently the presymplectic manifold $\left(Y_{\alpha}, \omega_{\alpha}\right)$ is obstructed.

Remark 12.1. In the irrational case of $\alpha$, the flow of $X_{H_{\alpha}}$ does not generate a circle action, and there is no simple analog of the second projection

$$
\pi_{2}: Y_{\alpha} \rightarrow Y_{\alpha} / S_{H_{\alpha}}^{1} \text {. }
$$

Analysis of the first cohomology $H^{1}\left(Y_{\alpha}, \omega_{\alpha}\right)$ for the irrational case requires a fair amount of general foliation theory, whose study we will postpone to a separate paper elsewhere.

\section{Appendix: Description in the super or GRADED LANGUAGE}

In this appendix, we will provide a more physical description of our deformation problem in the context of Batalin-Vilkovisky formalism of supermanifolds. Because of this, we will not attempt to make our discussion completely rigorous in the mathematical sense in this appendix. However most of the discussions except few explicitly stated conjectures can be made mathematically rigorous, which we postpone to a future work. We already gave one such example in the proof of Theorem 9.4 in section 8 .

We will also describe the formal deformation space of the strong homotopy Lie algebra $\mathfrak{l}_{(Y, \omega ; \Pi)}^{\infty}$. We refer readers to $[\mathrm{OP}]$ for more explanation of the BV formalism and for an off-shell description of the $A$-model of topological sigma models. We refer to [AKSZ] for a similar approach to the closed $A$-model (the Gromov-Witten theory) on Kähler manifold.

We shall interpret general open string $A$-model as a machine to quantize the algebra of functions on $\mathbb{L}=\Pi E$ as an $A_{\infty}$-algebra or, equivalently, as an 1-algebra in the sense of Kontsevich [K2]. First we review the basic set up for the quantization of the 1-algebra presented as in [OP], following [P1], [P2]. Then we shall show how a coisotropic submanifold naturally arises as the general boundary condition 
for the open string $A$-model and that deformations of the coisotropic submanifold correspond to boundary deformations of the open string $A$-model.

Let $\mathbb{L}$ be a smooth $\mathbb{Z}$-graded space over $\mathbb{C}$ and let $\mathfrak{l}$ be the super-commutative ring of functions on $\mathbb{L}$. As an abelian group we have a direct sum decomposition $\mathfrak{l}=\oplus_{n} \mathfrak{l}_{n}$, where $\mathfrak{l}_{n}$ is the maximal subspace of $\mathfrak{l}$ consisting of degree $n$ functions. In this appendix, we will use the complex without shift of grading. Then we have supercommutative (and associative) product with degree 0 such that, for homogenous element $\gamma_{1}, \gamma_{2} \in \mathfrak{l}$

$$
\gamma_{1} \gamma_{2}-(-1)^{\left|\gamma_{1}\right|\left|\gamma_{2}\right|} \gamma_{2} \gamma_{1}=0
$$

where $|\gamma|$ denotes the parity of $\gamma$ defined by degree $(\gamma) \bmod 2$. Let $T^{*}[1] \mathbb{L}$ be the total space of the twisted by degree 1 cotangent bundle to $\mathbb{L}$ and let $\mathfrak{t}$ be the super-commutative (and associative) ring of functions on it. We have a direct sum decompositions $\mathfrak{t}=\oplus_{\ell} \mathfrak{t}_{\ell}$ of $\mathcal{C}$ as an abelian group and super-commutative (and associative) product with degree 0 . Note that there is an odd symplectic structure $\Omega$ on $T^{*}[1] \mathbb{L}$ induced from the canonical symplectic structure of the total space of tangent bundle $T^{*} \mathbb{L}$ to $\mathbb{L}$

Definition 13.1. The pair $(\mathfrak{t},[\bullet, \bullet])$, where $[\bullet, \bullet]: \mathfrak{t}_{\ell_{1}} \otimes \mathfrak{t}_{\ell_{2}} \rightarrow \mathfrak{t}_{\ell_{1}+\ell_{2}-1}$ is the graded Poisson bracket of degree -1 induced from the canonical symplectic form $\Omega$ of degree 1 on $T^{*}[1] \mathbb{L}$, is called the structure of symplectic 2-algebra on $T^{*}[1] \mathbb{L}$ or on t. For any homogeneous elements $A, B, C \in \mathfrak{t}$

(1) super-commutativity

$$
[A, B]=-(-1)^{(|A|+1)(|B|+1)}[B, A]
$$

(2) super-Jacobi

$$
[A,[B, C]]=[[A, B], C]+(-1)^{(|A|+1)(|B|+1)}[B,[A, C]]
$$

(3) super-Leibnitz

$$
[A, B \cdot C]=[A, B] C+(-1)^{(|A|+1)|B|} B[A, C]
$$

(4) linearity

$$
[A, B+C]=[A, B]+[A, C]
$$

Corollary 13.1. The symplectic 2-algebra $(\mathfrak{t},[\bullet, \bullet])$ is a Gerstenhaber algebra

Corollary 13.2. The bracket $[\bullet, \bullet]$, after forgetting the product, induces a structure of Lie algebra on $\mathfrak{t}_{1}$;

$$
[\bullet, \bullet]: \mathfrak{t}_{1} \otimes \mathfrak{t}_{1} \longrightarrow \mathfrak{t}_{1} .
$$

From the corollary above we have degree preserving adjoint action $\operatorname{ad}_{(-B)}(A)=$ $[A, B]$ by an element $B \in \mathfrak{t}_{1}$ on any homogeneous element $A \in \mathfrak{t}$ and the associated transformation

$$
e^{a d_{(-B)}}(A):=A+[A, B]+\frac{1}{2 !}[[A, B], B]+\ldots,
$$

The above does not make sense unless the Lie algebra $\mathfrak{t}^{1}$ is nilpotent, otherwise we may tensor it with suitable Artinian ring [Ge]. Then

$$
\left[e^{a d_{(-B)}}\left(A_{1}\right), e^{a d_{(-B)}}\left(A_{2}\right)\right]=e^{a d_{(-B)}}\left(\left[A_{1}, A_{2}\right]\right) .
$$

We remark that the adjoint action $e^{a d_{B}}$, for $B \in \mathfrak{t}_{1}$ is equivalent to a degree preserving canonical transformation connected to the identity. 
Note that any odd element in $\mathfrak{t}$ automatically commutes with itself on the bracket, while the condition that an even element commutes with itself on the bracket is non-trivial.

We say there is a structure of weak homotopy Lie 1-algebroid on $\mathfrak{l}$ or on $\mathbb{L}$ if there exist a non-vanishing element $H \in \mathfrak{t}_{2}$ satisfying $[H, H]=0$. Two structures $H$ and $H^{\prime}$ of weak homotopy Lie 1-algebroid on $\mathbb{L}$ are defined to be equivalent if there exists some $B \in \mathfrak{t}^{1}$ such that $H^{\prime}=e^{a d_{B}}(H)$.

Consider a natural $\mathbb{C}^{*}$ action of weight 1 on the fiber, over the zero section $\mathbb{L}$, of $T^{*}[1] \mathbb{L}$ such that the degree 1 symplectic form $\Omega$ on $T^{*}[1] \mathbb{L}$ has weight 1 . For given $H$ above, we may expand it as

$$
H=\sum_{\ell=0}^{\infty} H_{\ell}
$$

in the neighborhood of $\mathbb{L}$ according to the integral weight $n$ of the $\mathbb{C}^{*}$ action. We may identify $H_{0}$ above as the restriction $\left.H\right|_{\mathbb{L}}$ of $H$ to $\mathbb{L}$.

A structure of strong homotopy Lie 1-algebroid on $\mathbb{L}$ or on $\mathfrak{l}$ is defined by an element $H \in \mathfrak{t}_{2}$ satisfying

$$
[H, H]=0,\left.\quad H\right|_{\mathbb{L}}=0 .
$$

Consider a structure $H$ of strong homotopy Lie 1-algebroid on $\mathbb{L}$ which has a decomposition

$$
H=\sum_{\ell=1}^{\infty} H_{\ell}
$$

according to the weights of the representation of $\mathbb{C}^{*}$ mentioned above. The equation $[H, H]=0$ has the corresponding decompositions

$$
\begin{aligned}
{\left[H_{1}, H_{1}\right] } & =0, \\
{\left[H_{1}, H_{2}\right] } & =0, \\
\frac{1}{2}\left[H_{2}, H_{2}\right]+\left[H_{1}, H_{3}\right] & =0, \\
\vdots &
\end{aligned}
$$

etc. For a given $H_{n}$ in the sequence $\left(H_{1}, H_{2}, \ldots\right)$ of above we can associate $n$-multilinear map $\mathfrak{m}_{n}$ of degree $2-n$;

$$
\mathfrak{m}_{n}: \mathfrak{l} \otimes n \longrightarrow \mathfrak{l}
$$

by, for any set $\gamma_{1}, \ldots, \gamma_{n}$ of homogenous elements of $\mathfrak{l}$

$$
\mathfrak{m}_{n}\left(\gamma_{1}, \ldots, \gamma_{n}\right):=\left[\left[\cdots\left[H_{n}, \gamma_{1}\right], \cdots\right], \gamma_{n}\right]
$$

Then the relation (13.1) together with the super-Jacobi identity of the bracket $[\bullet, \bullet]$ implies that $\left(\mathfrak{m}_{1}, \mathfrak{m}_{2}, \mathfrak{m}_{3}, \ldots\right)$ satisfies the relation equivalent to that of strong homotopy Lie algebra $\left(L_{\infty}\right.$-algebra in short).

Remark 13.2. Our definition includes the standard notion of the Lie algebroid as a special case, as shown below. Consider a smooth manifold $M$ and a vector bundle $E$ over $M$. Let $\mathbb{L}=\Pi E$ be the total space of $E$ after applying twisting functor $\Pi$ by degree 1 to the fiber. Let $\left\{x^{I}\right\}$ be local coordinates on $X,\left\{e_{a}\right\}$ be a local frame on $E$ and $\left\{e^{\alpha}\right\}$ be the dual frame. Then we may identify $\left\{x^{I}, c^{\alpha}:=\Pi e^{\alpha}\right\}$ as a coordinate 
system on $\Pi E$. Consider $T^{*}[1] \mathbb{L}$ with Darboux coordinates $\left\{x^{I}, c^{\alpha} \mid \chi_{I}, e_{\alpha}\right\}$ with degree $\{0,1 \mid 1,0\}$ and the canonical degree 1-symplectic structure

$$
\Omega=d \chi_{I} d x^{I}+d e_{\alpha} d c^{\alpha} .
$$

Now consider $H \in \mathfrak{t}_{2}$ given by

$$
H=c^{\alpha} \Gamma(x)_{\alpha}{ }^{I} \chi_{I}+\frac{1}{2} C(x)_{\alpha \beta}{ }^{\gamma} c^{\alpha} c^{\beta} e_{\gamma}
$$

Then the condition $[H, H]=0$ means that $\left(\Gamma_{\alpha}{ }^{I}, C_{\alpha \beta}{ }^{\gamma}\right)$ are the anchor and structure function of Lie algebroid. Note that $\left.H\right|_{\mathbb{L}}=0$. Let $\sigma=s^{\alpha} e_{\alpha}$ and $\tau=\tau^{\alpha} e_{\alpha}$ by any sections of $E$ and let $g$ be a smooth function on $M$ then

$$
[[H, \sigma], g \tau]=\sigma^{\alpha} \Gamma_{\alpha}{ }^{I} \frac{\partial g}{\partial x^{I}}+g \cdot C_{\alpha \beta}^{\gamma} \sigma^{\alpha} \tau^{\beta} e_{\gamma} .
$$

The graded supercommutative algebra $\mathfrak{l}$ functions on $\mathbb{L}$ is isomorphic the exterior algebra of $\mathrm{E}$ differential forms. The corresponding $\mathrm{E}$ differential operator is given by $\mathcal{Q}=[H, \ldots]$ restricted to $\mathbb{L}$;

$$
\left.\mathfrak{m}_{1} \equiv \mathcal{Q}_{1}\right|_{\mathbb{L}}=c^{\alpha} \Gamma_{\alpha}{ }^{I} \frac{\partial}{\partial x^{I}}+\frac{1}{2} C_{\alpha \beta}{ }^{\gamma} c^{\alpha} c^{\beta} \frac{\partial}{\partial c^{\gamma}} .
$$

Note that $H=H_{1}$ and, thus, $\mathfrak{m}_{2}=\mathfrak{m}_{3}=\ldots=0$.

Two structures $H$ and $H^{\prime}$ of strong homotopy Lie 1-algebroid on $\mathbb{L}$ are equivalent if they are related by the adjoint action of $\beta \in \mathfrak{t}_{1}$ satisfying $\left.\beta\right|_{\mathbb{L}}=0$. Note that such an adjoint action preserves the conditions $\left.H\right|_{\mathbb{L}}=\left.H^{\prime}\right|_{\mathbb{L}}=0$, and equivalent to a change of Lagrangian complimentary in $T^{*}[1] \mathbb{L}$.

Let $\Gamma \in \mathfrak{l}_{1} \subset \mathfrak{t}_{1}$ and let $H^{\Gamma} \equiv e^{a d_{\Gamma}}(H)$ denotes the resulting canonical transformations of $H$. Then it is obvious that $\left[H^{\Gamma}, H^{\Gamma}\right]=0$, while $\left.H^{\Gamma}\right|_{\mathbb{L}} \neq 0$ in general. For each $\Gamma$ leading to $\left.H^{\Gamma}\right|_{\mathbb{L}}=0$ we have another structure of strong homotopy Lie 1-algebroid on $\mathbb{L}$. The condition $\left.H^{\Gamma}\right|_{\mathbb{L}}=0$ is equivalent to Maurer-Cartan equation

$$
\sum_{\ell=1}^{\infty} \frac{1}{\ell !} \mathfrak{m}_{\ell}(\Gamma, \ldots, \Gamma)=0
$$

Now we explain how the above general story is relevant to our subject.

We consider a symplectic manifold $\left(X, \omega_{X}\right)$ and the graded space $T[1] X$, which is the total space of twisted by the degree 1 tangent bundle to $X$ where $U$ is the degree or the ghost number. Let $\mathfrak{t}=\oplus_{k=0}^{2 n} \mathfrak{t}_{k}$ be the $\mathbb{Z}$-graded supercommutative algebra of smooth functions on $T[1] X$. The algebra $t$ is isomorphic to the exterior algebra of differential forms on $X$, such that $\Omega^{k}(X) \simeq \mathfrak{t}_{k}$ and the wedge product is replaced with the supercommutative product. The exterior derivative induces an odd degree 1 vector field $\mathcal{Q}$ on $T[1] X$ such that $\mathcal{Q}: \mathfrak{t}_{k} \rightarrow \mathfrak{t}_{k+1}$ and $\mathcal{Q}^{2}=0$. Thus we have a structure of differential graded algebra $(\mathfrak{t}, \mathcal{Q}, \cdot)$. The cohomology of $(\mathfrak{t}, \mathcal{Q}, \cdot)$ is isomorphic to de Rham cohomology of $X$. The symplectic structure $\omega_{X}$ on $X$ induces a degree 1 (odd) symplectic form $\Omega$ on $T[1] X$ via the standard isomorphism $T X \rightarrow T^{*} X$ together with the twisting. Thus we have a structure of symplectic 2-algebra on $T[1] X$ by the pair $(\mathfrak{t},[\bullet, \bullet])$, we the degree -1 (odd) Poisson bracket is defined by $\Omega$. The above bracket is equivalent to Koszul bracket, which is the covariant version Schouten-Nijenhuis bracket [Ko]. 
There exists an element $H \in \mathfrak{t}_{2}$, which is isomorphic to the symplectic form $\omega_{X}$. Using the closedness of $\omega$, it is not difficult to check the following identities

$$
[H, H]=0, \quad \mathcal{Q}=[H,]
$$

Thus $\mathcal{Q}$, isomorphic to the exterior derivative, is realized as the Hamiltonian vector field of the function $H$.

Remark 13.3. It is instructive to give a coordinate representation of above. We introduce a local coordinates $\left\{x^{I}\right\}, I=1, \ldots, 2 n$, on $X$. We denote the corresponding fiber coordinates on $T[1] X$ by $\left\{\psi^{I}\right\}$ carrying the degree 1 . Then $\mathcal{Q}=\psi^{I} \frac{\partial}{\partial x^{I}}$. In the sense of ordinary geometry, $\psi^{I}$ is nothing but $d x^{I}$ considered as a fiberwise linear function on $T[1] X$. Now the symplectic structure $\omega=\frac{1}{2} \omega_{I J} d x^{I} \wedge d x^{J}$ on $X$ induces a non-degenerate function

$$
H=\frac{1}{2} \omega_{I J} \psi^{I} \psi^{J} \in \mathfrak{t}_{2}
$$

of degree 2 on $T[1] X$. Thus the condition $d \omega_{X}=0$ is equivalent to $\mathcal{Q} H=0$. The symplectic structure $\omega_{X}$ on $X$ induces a degree $U=1$ symplectic form

$$
\Omega=d\left(\omega_{I J} \psi^{I}\right) d x^{J}
$$

on $T[1] X$ and such that the corresponding graded Poisson bi-vector on $T[1] X$ is given by

$$
\Omega^{*}=\left[\left(\omega^{-1}\right)^{I J} \frac{\partial}{\partial \psi^{I}} \wedge \frac{\partial}{\partial x^{J}}+\frac{1}{2}\left(\frac{\partial\left(\omega^{-1}\right)^{I J}}{\partial x^{K}}\right) \psi^{K} \frac{\partial}{\partial \psi^{I}} \wedge \frac{\partial}{\partial \psi^{J}}\right.
$$

which define the graded Poisson bracket $[\bullet, \bullet]$. One may check $[H, H]=0$ and $\mathcal{Q}=[H, \bullet]$ by an explicit computation.

Let $\mathbb{L}$ be a Lagrangian subspace of $(T[1] X, \Omega)$. Then the following is easy to see from the form of the Hamiltonian $H$ in coordinates.

Lemma 13.3. Any (conic) Lagrangian subspace $\mathbb{L}$ satisfying $\left.H\right|_{\mathbb{L}}=0$ is equivalent to $\Pi E=\Pi(T Y)^{\omega}$, where $Y$ is a coisotropic submanifold of $X$ and $\Pi E$ is the total space of $E$ after twisting the fiber by 1.

Consequently each coisotropic submanifold $Y \subset X$ inherits a structure of strong homotopy Lie 1-algebroid induced from the symplectic structure of $X$, and the (formal) deformation problem of coisotropic submanifold is equivalent to that of strong homotopy Lie 1-algebroid. We remark that $\mathfrak{l}=\oplus \mathfrak{l}_{\bullet}$ is isomorphic to $\oplus \Gamma\left(\wedge^{\bullet} E^{*}\right)$ and in particular $\Gamma \in \mathfrak{l}_{1}$ is isomorphic to $\Gamma\left(E^{*}\right)$. The condition $\left.H^{\Gamma}\right|_{\mathbb{L}}=0$ can be identified with $\left.H\right|_{\mathbb{L}^{\Gamma}}=0$ where $\mathbb{L}^{\Gamma}$ is the graph of Lagrangian subspace generated by $\Gamma$. Thus the condition $\left.H^{\Gamma}\right|_{\mathbb{L}}=0$ means $\mathbb{L}^{\Gamma}$ is given by $\Pi E^{\prime} \equiv \Pi\left(T Y^{\prime}\right)^{\omega}$, where $Y^{\prime}$ is another coisotropic submanifold.

It is also natural to consider extended deformations of coisotropic submanifold. Let us consider a graded Artin ring with maximal ideal $\mathfrak{a}$;

$$
\mathfrak{a}=\bigoplus_{-(n-k) \leq j \leq 1} \mathfrak{a}_{j}
$$

where $(n-k)$ is the rank of $E=(T Y)^{\omega}$. Let $\Upsilon \in(\mathfrak{l} \otimes \mathfrak{a})_{1}$. Then the condition

$$
\left.H^{\Upsilon}\right|_{\mathbb{L}} \equiv a d_{\Upsilon}(H) \equiv \sum_{n=1}^{\infty} \frac{1}{\ell !} m_{\ell}(\Upsilon, \ldots, \Upsilon)=0
$$


may be regarded as the condition for the extended deformations of coisotropic submanifold $Y$.

We note that the deformation problem of coisotropic submanifold is obstructed in general. On the other hand our setting allows us to consider an extended deformation problem allowing both $\Gamma$ and $H$ to vary. It may turn out that this extended deformation problem could be unobstructed. This is a subject of future study.

Now we like to motivate the problem of quantization of coisotropic submanifold. We begin with recalling a lemma of Kontsevich [K2] stating

The cohomology of the Hochschild complex of the algebra of functions $A_{1}(\mathbb{L})$ on $\mathbb{L}$, regarded as an 1-algebra, is isomorphic to the space $\mathfrak{t}$ of functions on $T^{*}[1] \mathbb{L}$.

In the spirit of the above lemma, we may regard a structure $H$ of strong homotopy Lie 1-algebroid is an element of the 1st cohomology of Hochschild complex of $A_{1}(\mathbb{L})$, which is the 1st order deformations of $A_{1}(\mathbb{L})$ as a strong homotopy associative $\left(A_{\infty}\right.$ in short) algebra (or simply as an 1-algebra). In the spirit of Kontsevich's formality theorem [K1], we may define the quantization of the 1-algebra $A_{1}(\mathbb{L})$ as a quasiisomorphism between the Hochschild complex of $A_{1}(\mathbb{L})$ and its cohomology.

Open string or quantum field theory in a two-dimensional manifold with boundary may be regarded as a universal machine to quantize the 1-algebra. The following can be shown

For each structure $H$ of the strong homotopy Lie 1-algebroid on any $\mathbb{L}$ there exists a pre-quantum field theory in two-dimension with boundary, whose boundary condition is defined in terms of $\mathbb{L}$, depending on the topology of two-manifolds, so that it satisfies the classical BV master equation.

In particular the set of solutions of (13.2), modulo equivalence, is isomorphic to the moduli space of boundary interactions.

The second named author [P2] called such a QFT as an open 1-braneoid. Assume that the resulting QFT actually satisfies the quantum BV master equation. Then a conjecture is that the path integral generates a quasi-isomorphism between the Hochschild complex of $A_{1}(\mathbb{L})$ and its cohomology.

Now we may interpret the open string $A$-model with the coisotropic boundary condition as a machine to quantize the algebra of functions on $\mathbb{L}=\Pi E$ as an $A_{\infty}$ algebra. We may simply call the problem as quantization of a coisotropic submanifold $Y$ on a symplectic manifold $X$. In [OP] we shall see that the genus zero open string $A$-model governs the maps $\Phi: T[1] D \rightarrow T[1] X$ satisfying $\Phi(T[1](\partial D)) \subset$ $\mathbb{L}=\Pi E$. Then the path integral is formally defined as an integral over an (infinite dimensional) Lagrangian subspace of the space all maps $\Phi$ determined by conformal structure on $D$ and an almost complex structure on $X$.

Example 13.4. Let $Y=X$, then $\mathbb{L}=X$ and $m_{\ell} \neq 0$ only for $\ell=2$ such that $m_{2}$ is the usual Poisson bracket on $X$, and consider the zero-instanton sector governed by constant maps of the $A$-model. Then the quantization problem is nothing but that of $X$ as a Poisson manifold. This is the original context of Kontsevich's formality theorem [K1], as interpreted in the path integral approach of [CF1]. What is the result after including instanton corrections even in this case? 
We conjecture, which can be justified at the physical level of rigor, that the Hochschild complex of $A_{1}(\mathbb{L}=\Pi E)$ is quasi-isomorphic to its cohomology. The above conjecture is based on the following fact.

There exist a degree -1 odd differential operator $\Delta ; \Delta: \mathfrak{t}_{\ell} \rightarrow \mathfrak{t}_{\ell-1}$ satisfying $\Delta^{2}=0$ and generates the bracket $[\bullet, \bullet]$ such that

$$
\Delta H=\Delta H^{\Upsilon}=0 .
$$

The above properties can be used to formally show that the open string $A$-model with coisotropic boundary condition actually satisfies the quantum BV master equation, which implies that the path integral would give the formality map. The rigorous proof of this assertion will be investigated in the future.

Now we go back to our study of coisotropic submanifolds using this BV formalism and give a proof of Theorem 9.4 in this context. We introduce a local coordinates system $\left(y^{i}, q^{\alpha} \mid p_{\alpha}\right)$ of $X$ in a neighborhood of $Y$ as in section 4. It was shown in section 4 that the symplectic form $\omega$ in $X$ can be written as

$$
\begin{aligned}
\omega= & \frac{1}{2}\left(\omega_{i j}-p_{\beta} F_{i j}^{\beta}\right) d y^{i} \wedge d y^{j} \\
& -\left(d p_{\beta}+p_{\beta} \frac{\partial R_{i}^{\beta}}{\partial q^{\gamma}} d y^{i}\right) \wedge\left(d q^{\delta}-R_{j}^{\delta} d y^{j}\right)
\end{aligned}
$$

It is straightforward to derive

$$
\begin{aligned}
\widetilde{\omega}\left(\frac{\partial}{\partial q^{\delta}}\right)= & d p_{\delta}+p_{\beta} \frac{\partial R_{i}^{\beta}}{\partial q^{\delta}} d y^{i} \\
\widetilde{\omega}\left(\frac{\partial}{\partial p_{\delta}}\right)= & -\left(d q^{\delta}-R_{j}^{\delta} d y^{j}\right) \\
\widetilde{\omega}\left(\frac{\partial}{\partial y^{i}}\right)= & \left(\omega_{i j}-p_{\beta} F_{i j}^{\beta}\right) d y^{j}-p_{\beta} \frac{\partial R_{i}^{\beta}}{\partial q^{\delta}}\left(d q^{\delta}-R_{j}^{\delta} d y^{j}\right) \\
& -R_{i}^{\delta}\left(d p_{\delta}+p_{\beta} \frac{\partial R_{i}^{\beta}}{\partial q^{\delta}} d y^{i}\right)
\end{aligned}
$$

The last identity can be rewritten as

$$
\widetilde{\omega}\left(e_{i}\right)=\widetilde{\omega}_{i j} d y^{j}
$$

where we recall to have defined

$$
e_{i}=\frac{\partial}{\partial y^{i}}+R_{i}^{\delta} \frac{\partial}{\partial q^{\delta}}-p_{\beta} \frac{\partial R_{i}^{\beta}}{\partial q^{\delta}} \frac{\partial}{\partial p_{\delta}} .
$$

Combining these we have derived the following formula for the inverse $(\widetilde{\omega})^{-1}$ : $T^{*} X \rightarrow T X:$

$$
\left\{\begin{array}{l}
\widetilde{\omega}^{-1}\left(d y^{i}\right)=\widetilde{\omega}^{i j} e_{j} \\
\widetilde{\omega}^{-1}\left(d p_{\delta}\right)=\frac{\partial}{\partial q^{\delta}}-p_{\beta} \frac{\partial R_{i}^{\beta}}{\partial q^{\delta}} \widetilde{\omega}^{i j} e_{j} \\
\widetilde{\omega}^{-1}\left(d q^{\delta}\right)=-\frac{\partial}{\partial p_{\delta}}-R_{j}^{\delta} \widetilde{\omega}^{i j} e_{j}
\end{array}\right.
$$

In the canonical coordinates of $T[1] X$ associated $\left(y^{i}, q^{\alpha} \mid p_{\alpha}\right)$, the even Hamiltonian $H$ becomes

$$
H=\frac{1}{2}\left(\omega_{i j}-p_{\beta} F_{i j}^{\beta}\right) \psi^{i} \psi^{j}+\left(\eta^{\delta}-R_{j}^{\delta} \psi^{j}\right)\left(\chi^{\delta}+p_{\beta} \frac{\partial R_{i}^{\beta}}{\partial q^{\delta}} \psi^{i}\right)
$$


It would be more convenient to write down the Hamiltonian on $T^{*} \mathbb{L} \cong T[1] X$ near $\mathbb{L}=(T Y)^{\omega}[1] \subset T[1] X$ using the super version of Darboux-Weinstein theorem. We denote the (super) canonical coordinates of $T^{*}[1] \mathbb{L}$ associated with $\left(y^{i}, q^{\alpha} \mid p_{\alpha}\right)$ by

$$
\left(\begin{array}{lll}
y^{i}, & q^{\alpha} & \mid p_{*}^{\alpha} \\
y_{i}^{*}, & q_{\alpha}^{*} & \mid p_{\alpha}
\end{array}\right)
$$

Here we note that the degree of $y^{i}, q^{\alpha}$ and $p_{\alpha}$ are 0 while their anti-fields, i.e., those with $*$ in them have degree 1 . And we want to emphasize that $\mathbb{L}$ is given by the equation

$$
y_{i}^{*}=p_{\alpha}=p_{*}^{\alpha}=0
$$

and $\left(y^{i}, y_{i}^{*}\right),\left(p_{\alpha}, q_{\alpha}^{*}\right)$ and $\left(p_{*}^{\alpha}, q^{\alpha}\right)$ are conjugate variables. Then we have the canonical odd symplectic form of degree 1 and associated canonical odd Poisson bracket $[\bullet, \bullet]^{*}$ of degree -1 .

It follows from (13.5) that we have

$$
\begin{aligned}
\widetilde{\omega}^{-1}\left(d q^{\delta}-R_{j}^{\delta} d y^{j}\right) & =-\frac{\partial}{\partial p_{\delta}} \\
\widetilde{\omega}^{-1}\left(d p_{\delta}+p_{\beta} \frac{\partial R_{j}^{\beta}}{\partial q^{\delta}} d y^{j}\right) & =\frac{\partial}{\partial q^{\delta}}
\end{aligned}
$$

and so that $H$ has the form

$$
H=\frac{1}{2} \widetilde{\omega}^{i j} y_{i}^{\#} y_{j}^{\#}+p_{*}^{\delta} q_{\delta}^{*}
$$

in the canonical coordinates of $T^{*}[1] \mathbb{L}$. Here we define $y_{i}^{\#}$ to be

$$
y_{i}^{\#}:=y_{i}^{*}+R_{i}^{\delta} p_{*}^{\delta}-p_{\beta} \frac{\partial R_{i}^{\beta}}{\partial q^{\delta}} q_{\delta}^{*} .
$$

We then derive

$$
\left.\widetilde{\omega}_{\alpha}^{-1}=\omega_{\pi(\alpha)}^{-1} \sum_{\ell=0}^{\infty}\left(F^{\#}\right\rfloor \alpha\right)^{\ell} \quad \text { on } T Y / E
$$

(See (11.2) in section 11]later.) which is written as

$$
\widetilde{\omega}_{\alpha}^{i j}=\omega_{\pi(\alpha)}^{i j_{0}} \sum_{\ell=0}^{\infty}\left(p_{\beta_{1}} F_{j_{0}}^{\beta_{1} j_{1}}\right)\left(p_{\beta_{2}} F_{j_{1}}^{\beta_{2} j_{2}}\right) \cdots\left(p_{\beta_{\ell}} F_{j_{\ell-1}}^{\beta_{\ell} j_{\ell}}\right)
$$

in coordinates where $\alpha=p_{\beta} f_{\beta}^{*}$. Since $H$ vanishes on $\mathbb{L}$, its Hamiltonian vector field $\mathcal{Q}$ is tangent to $\mathbb{L}$ and so can be canonically restricted to $\mathbb{L}$ as an odd vector field on $\mathbb{L}$. The odd vector field

$$
m_{1}=\left.\mathcal{Q}\right|_{\mathbb{L}}
$$

acts on

$$
\mathfrak{l}=\bigoplus_{\ell=0}^{n-k} \mathfrak{l}^{\ell} \cong \bigoplus_{\ell=0}^{n-k} \Omega^{\ell}(\mathcal{F})
$$

as the corresponding directional derivative and equivalent to $d_{\mathcal{F}}$.

The general discussions mentioned above then implies that the strong homotopy Lie algebroid structure is obtained by expanding $H$ into the series

$$
H=\sum_{\ell=1} H_{\ell}, \quad H_{\ell} \in \mathfrak{l}^{\ell}
$$


in the normal direction of $\mathbb{L}$, i.e., in terms of $\left(y_{i}^{*}, p_{*}^{\alpha}, p_{\alpha}\right)$ and use the odd Poisson bracket $[\bullet, \bullet]$.

Now Theorem 9.4 immediately follows from our general discussions.

\section{REFERENCES}

[AKSZ] Alexandrov, M., Kontsevich, M., Schwarz, A. and Zaboronsky, O., The geometry of the master equation and topological quantum field theory, Int. J. Mod. Phys. A12 (1997), 1405-1430

[CF1] Cattaneo, A.S. and Felder, G., A path integral approach to the Kontsevich quantization formula, Comm. Math. Phys., 212 (2000), 591-611.

[CF2] Catteneo, A.S. and Felder, G., Coisotropic submanifolds in Poisson geometry and branes in the Poisson sigma model, preprint, math.QA/0309180

[CW] Cannas da Silva, A. and Weinstein, A. Geometric Models for Noncomutative Algebras, Berkeley Math. Lec. Notes, 10, AMS, Providence, RI, 1999.

[Fu] Fukaya, K., Deformation theory, homological algebra and mirror symmetry, in "Geometry and Physics of Branes", Series in High Energy Physics-Cosmology and Gravitation, eds. by Bruzzo, U., Gorini, V. and Moschella, U., pp 121 - 209, Institute of Physics Publishing, Bristol 2003.

[FOOO] Fukaya, K., Oh, Y.-G., Ono, K. and Ohta, H., Lagrangian intersection Floer theory anomaly and obstruction, preprint, 2000.

[GD] Gelfand, I. and Dorfman, I. Schouten bracket and Hamiltonian operators, Funcktsional. Anal. i Prilozhen 14 (1980), 71 -74.

[Ge] Gerstenhaber, M., On the deformation of rings and algebras, Ann. of Math. (2) 79 (1964), 59-103.

[Gz] Getzler, E., A Darboux theorem for Hamiltonian operators in the formal calculus of variations, Duke Math. J. 111 (2002), 535 - 560.

[GM] Goldman, W. and Millson, J., The deformation theory of representation of fundamental groups in compact Kähler manifolds, Publ. Math. IHES 67 (1988), 43-96.

[Go] Gotay, M., On coisotropic imbeddings of pre-symplectic manifolds, Proc. Amer. Math. Soc., 84 (1982), 111-114.

[Ha] Haefliger, A., Some remarks on foliations with minimal leaves, J. Differ. Geom. 15 (1980), 269 - 284.

[KaOr] Kapustin, A. and Orlov, D., Remarks on A-branes, mirror symmetry and the Fukaya category, J. Geom. Phys. 48 (2003), no. 1, 84-99, hep-th/0109098

[K1] Kontsevich, M., Deformation quantization of Poisson manifolds, Lett. Math. Phys. 66 (2003), no. 3, 157-216, QA/9709040.

[K2] Kontsevich, M., Operads and motives in deformation quantization, Lett. Math. Phys. 48 (1999), 35-72, QA/9904055.

[Ko] Koszul, J.L., Crochet de Schouten-Nijenhuis et cohomologie, Asterisque, (1985) 251.

[Mo] Molino, P., Riemannian Foliations, Birkhäuser, Boston, 1988.

[MM] Moerdijk, I. and Mrcun, J., Introduction to Foliations and Lie Groupoids, Cambridge Press, 91, 2003.

[NT] Nest, R., Tsygan, B. Deformations of symplectic Lie algebroids, deformations of holomorphic symplectic structures, and index theorems, Asian J. Math. 5 (2001), no. 4, 599-635, QA/9906020

[Oh] Oh, Y.-G., Geometry of coisotropic submanifolds in symplectic and Kähler manifolds, preprint, math.SG/0310482

[OP] Oh, Y.-G. and Park, J.-S., Open/Closed A-model, in preparation.

[P1] Park, J.-S., Topological open p-branes, Proceedings of the KIAS conference "Symplectic Geometry and Mirror Symmetry", pp 311-384, eds. by Fukaya, Oh, Ono and Tian, World Scientific, 2001, hep-th/0012141

[P2] Park, J.-S., Pursuing the quantum worlds; flat family of QFTs and quantization of $d$ algebras, preprint, hep-th/0308130

[R] Ruan, W.-D., Deformation of integral coisotropic submanifolds in symplectic manifolds, preprint, math.SG/0312107

[Sa] Satake, I., On a generalization of the notion of manifold, J. Math. Soc. Japan, 9 (1957), 464-492. 
[To] Tondeur, P. Geometry of Foliations, Monograph in Math. 90, Birkhäuser, Basel, 1997.

[V] Vaisman, I., Geometric quantization on presymplectic manifolds, Mh. Math. 96 (1983), 293-310.

[We1] Weinstein, A., Symplectic manifolds and their Lagrangian submanifolds, Advances in Math. 6 (1971), 329-346.

[We2] Weinstein, A., Coisotropic calculus on Poissorn manifolds, J. Math. Soc. Japan, 40 (1988), 705-727.

[We3] Weinstein, A., Symplectic V-manifolds, periodic orbits of Hamiltonian systems, and the volume of certain Riemannian manifolds, Comm. Pure Appl. Math. 30 (1977), 265-271.

[Za] Zambon, M., Neighborhoods of coisotropic submanifolds, preprint, 2002.

Department of Mathematics, University of Wisconsin, Madison, Wi 53706 \& Korea Institute for Advanced Study, 207-43 Cheongryangri-dong Dongdaemun-gu, Seoul 130-012, KOREA, oh@math.wisc.edu; Korea Institute for Advanced Study, 207-43 CheongryangriDong Dongdaemun-Gu, Seoul 130-012, KOREA, JAesuk@Kias.Re.Kr 